\title{
Wa ${ }^{a}$ e phenomena \\ analysis and numerics
}

\section{Co-dimension one stable blowup for the supercritical cubic wave equation}

Irfan Glogić, Birgit Schörkhuber

CRC Preprint 2018/39, November 2018

\section{KARLSRUHE INSTITUTE OF TECHNOLOGY}

\section{CRC 1173}

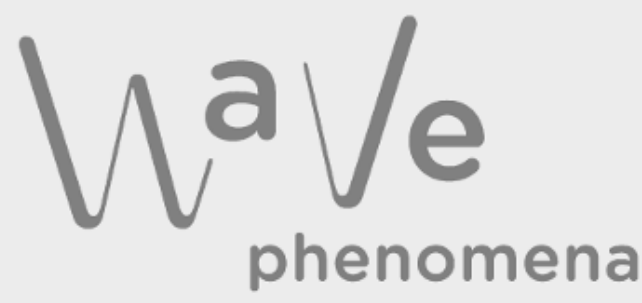




\section{Participating universities}

EBERHARD KARLS

UNIVERSITATT TUBINGEN

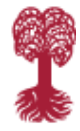

Funded by

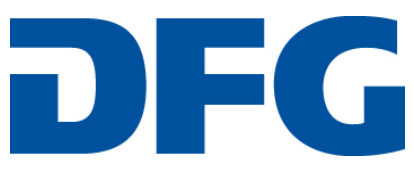

ISSN 2365-662X 


\title{
CO-DIMENSION ONE STABLE BLOWUP FOR THE SUPERCRITICAL CUBIC WAVE EQUATION
}

\author{
IRFAN GLOGIĆ AND BIRGIT SCHÖRKHUBER
}

\begin{abstract}
For the focusing cubic wave equation, we find an explicit, non-trivial self-similar blowup solution $u_{T}^{*}$, which is defined on the whole space and exists in all supercritical dimensions $d \geq 5$. For $d=7$, we analyze its stability properties without any symmetry assumptions and prove the existence of a co-dimension one Lipschitz manifold consisting of initial data whose solutions blowup in finite time and converge asymptotically to $u_{T}^{*}$ (modulo space-time shifts and Lorentz boosts) in the backward lightcone of the blowup point. The underlying topology is strictly above scaling.
\end{abstract}

\section{INTRODUCTION}

We consider the focusing wave equation

$$
\left(\partial_{t}^{2}-\Delta_{x}\right) u(t, x)=u(t, x)^{3}
$$

where $(t, x) \in I \times \mathbb{R}^{d}$ and $I \subset \mathbb{R}$ is an interval containing zero. The equation is invariant under the rescaling $u \mapsto u_{\lambda}$,

$$
u_{\lambda}(t, x)=\lambda^{-1} u(t / \lambda, x / \lambda)
$$

and leaves invariant the $\dot{H}^{s_{c}}\left(\mathbb{R}^{d}\right)-$ norm, $s_{c}=\frac{d}{2}-1$. Thus, the model is energy supercritical in $d \geq 5$ and we restrict ourselves to this case. It is well-known that Eq. (1.1) has solutions that blow up in finite time from smooth, compactly supported initial data

$$
u[0]=\left(u(0, \cdot), \partial_{t} u(0, \cdot)\right),
$$

see [34]. Locally, the stable blowup behavior for Eq. (1.1) is described by the ODE blowup

$$
u_{T}(t, x)=\frac{\sqrt{2}}{T-t},
$$

see [17], [19] and [10]. We note that $u_{T}$ is an example of a self-similar blowup solution with trivial spatial profile. For the supercritical focusing wave equation in the three-dimensional case it is well-known that non-trivial, smooth, self-similar solutions exist, see [7], and the result be extended to Eq. (1.1) for $d \geq 5$. However, in contrast to the three-dimensional case, where none of these solutions are known in closed form, Eq. (1.1) has the explicit self-similar solution

$$
u^{*}(t, x)=\frac{1}{t} U\left(\frac{|x|}{t}\right), \quad U(\rho)=\frac{2 \sqrt{2(d-1)(d-4)}}{d-4+3 \rho^{2}} .
$$

Irfan Glogić is supported by the Austrian Science Fund FWF, Project P 30076. Also, support from The Ohio State University Graduate School via Presidential Fellowship (during which a part of this work was completed) is gratefully acknowledged. Birgit Schörkhuber is funded by the Klaus-Tschira Stiftung and gratefully acknowledges financial support by the Deutsche Forschungsgemeinschaft (DFG) through CRC 1173 . 
To the best of our knowledge this solution has not been known so far. By using symmetries of the equation, we obtain a non-trivial self-similar blow up solution

$$
u_{T}^{*}(t, x)=(T-t)^{-1} U\left(\frac{|x|}{T-t}\right), \quad T>0,
$$

which is defined on all of $\mathbb{R}^{d}$ and becomes singular in forward time as $t \rightarrow T$. The aim of this paper is to investigate the stability properties of $u_{T}^{*}$ and to show that it has exactly one genuine unstable direction.

Recall, that Eq. (1.1) is invariant under shifts in space and time

$$
S_{T, x_{0}}(t, x):=\left(t-T, x-x_{0}\right)
$$

where $T \in \mathbb{R}$ and $x_{0}=\left(x_{0}^{j}\right)_{j=1 \ldots d} \in \mathbb{R}^{d}$, as well as under time reflection

$$
R(t, x):=(-t, x)
$$

and Lorentz transformations

$$
\Lambda(a):=\Lambda^{d}\left(a^{d}\right) \circ \Lambda^{d-1}\left(a^{d-1}\right) \circ \cdots \circ \Lambda^{1}\left(a^{1}\right)
$$

where $a=\left(a^{j}\right)_{j=1, \ldots, d} \in \mathbb{R}^{d}$ and the Lorentz boost in $j$-th direction $\Lambda^{j}\left(a^{j}\right)$ is given by

$$
\left\{\begin{aligned}
t & \mapsto t \cosh \left(a^{j}\right)+x^{j} \sinh \left(a^{j}\right) \\
x^{j} & \mapsto t \sinh \left(a^{j}\right)+x^{j} \cosh \left(a^{j}\right) \\
x^{k} & \mapsto x^{k} \quad(k \neq j) .
\end{aligned}\right.
$$

By composing these transformations we set

$$
\Lambda_{T, x_{0}}(a):=R \circ \Lambda(a) \circ S_{T, x_{0}} .
$$

This allows us to define a $(2 d+1)$-parameter family of solutions to Eq. (1.1)

$$
u_{T, x_{0}, a}^{*}(t, x):=u^{*} \circ \Lambda_{T, x_{0}}(a)(t, x) .
$$

Note that $(0,0)=\Lambda_{T, x_{0}}(a)\left(T, x_{0}\right)$ and the lightcones emanating from $\left(T, x_{0}\right)$ are mapped into the ones emanating from the origin. Hence, for $\left(t^{\prime}, x^{\prime}\right)=\Lambda_{T, x_{0}}(a)(t, x)$ we have the identity

$$
\left|x^{\prime}\right|^{2}-t^{\prime 2}=\left|x-x_{0}\right|^{2}-(t-T)^{2} \text {. }
$$

Also,

$$
t^{\prime}=A_{0}(a)(T-t)-A_{j}(a)\left(x^{j}-x_{0}^{j}\right)
$$

where

$$
\begin{aligned}
A_{0}(a) & :=\cosh \left(a^{1}\right) \cdots \cosh \left(a^{d}\right) \\
A_{1}(a) & :=\sinh \left(a^{1}\right) \cosh \left(a^{2}\right) \ldots \cosh \left(a^{d}\right) \\
A_{2}(a) & :=\sinh \left(a^{2}\right) \cosh \left(a^{3}\right) \ldots \cosh \left(a^{d}\right) \\
& \vdots \\
A_{d}(a) & :=\sinh \left(a^{d}\right)
\end{aligned}
$$

Now, we have the explicit form

$$
u_{T, x_{0}, a}^{*}(t, x)=\frac{1}{T-t} \psi_{a}^{*}\left(\frac{x-x_{0}}{T-t}\right),
$$


where

$$
\psi_{a}^{*}(\xi)=\frac{4 \gamma(\xi, a)}{2 \gamma(\xi, a)^{2}+|\xi|^{2}-1}
$$

with $\gamma(\xi, a)=A_{0}(a)-A_{j}(a) \xi^{j}$. For $a \in \mathbb{R}^{d}$ sufficiently small, $A_{0}(a)=1+O(|a|)$ and $A_{j}(a)=O(|a|)$ such that $\psi_{a}^{*} \in C^{\infty}\left(\mathbb{R}^{d}\right)$. From Eq. (1.5) and the scaling of Sobolev norms we obtain

$$
\left\|u_{T, x_{0}, a}^{*}(t, \cdot)\right\|_{\dot{H}^{k}\left(\mathbb{B}_{T-t}^{d}\left(x_{0}\right)\right)}=(T-t)^{s_{c}-k}\left\|\psi_{a}^{*}\right\|_{\dot{H}^{k}\left(\mathbb{B}^{d}\right)},
$$

for fixed $k \in \mathbb{N}_{0}$ and all $0 \leq t<T$, i.e., the solution blows up in the backward lightcone of $\left(T, x_{0}\right)$ for $k>s_{c}$. In the following, we restrict ourselves to the case $d=7$. We prove that there is a co-dimension one manifold of initial data in a small neighbourhood of $u_{1,0,0}^{*}$ that lead to blowup via $u_{T, x_{0}, a}^{*}$ in the backward lightcone of the blowup point.

Theorem 1.1. Let $d=7$ and define $\mathbf{h}:=\left(h_{1}, h_{2}\right)$ by

$$
h_{1}(x)=\frac{1}{\left(1+|x|^{2}\right)^{2}}, \quad h_{2}(x)=\frac{4}{\left(1+|x|^{2}\right)^{3}} .
$$

There exist constants $\omega>0, \delta>0$ and $c>0$ such that for all $\mathbf{v}=\left(v_{1}, v_{2}\right) \in C^{\infty}\left(\overline{\mathbb{B}_{2}^{7}}\right) \times$ $C^{\infty}\left(\overline{\mathbb{B}_{2}^{7}}\right)$, satisfying

$$
\|\mathbf{v}\|_{\mathcal{Y}}:=\left\|\left(v_{1}, v_{2}\right)\right\|_{H^{4} \times H^{3}\left(\mathbb{B}_{2}^{7}\right)} \leq \frac{\delta}{c}
$$

the following holds: There are parameters $a(\mathbf{v}) \in \overline{\mathbb{B}_{c \delta / \omega}^{7}}, x_{0}(\mathbf{v}) \in \overline{\mathbb{B}_{\delta}^{7}}, T(\mathbf{v}) \in[1-\delta, 1+\delta]$ and $\alpha(\mathbf{v}) \in[-\delta, \delta]$ depending Lipschitz continuously on $\mathbf{v}$ with respect to $\mathcal{Y}$ such that for initial data

$$
u[0]=u_{1,0,0}^{*}[0]+\mathbf{v}+\alpha(\mathbf{v}) \mathbf{h}
$$

there exists a unique solution $u$ to Eq. (1.1) on $\bigcup_{t \in[0, T)}\{t\} \times \overline{\mathbb{B}_{T-t}^{7}}$. Furthermore, $u$ blows up at $t=T, x=x_{0}$ and converges to $u_{T, x_{0}, a}^{*}$ according to

$$
(T-t)^{k-\frac{5}{2}}\left\|u[t]-u_{T, x_{0}, a}^{*}[t]\right\|_{\dot{H}^{k} \times \dot{H}^{k-1}\left(\mathbb{B}_{T-t}^{7}\left(x_{0}\right)\right)} \lesssim(T-t)^{\omega}
$$

for $k=1,2,3$.

Some remarks on the result are in order.

1. Assumptions on the initial data/Regularity of the solution. The assumption on the initial data, in particular the smallness of $\mathbf{v}$ in $\mathcal{Y}$, allows for an elementary proof of the Lipschitz continuity of the parameters $\left(a, T, x_{0}, \alpha\right)$. The same is expected to hold for $\mathbf{v}$ small in $H^{3} \times H^{2}\left(\mathbb{B}_{2}^{7}\right)$, but here more structure must be utilized and we will address this elsewhere. As in [18], the solution in Theorem 1.1 has to be understood as a lightcone solution, i.e., a solution of the abstract evolution equation corresponding to a reformulation of Eq. (1.1) in self-similar coordinates, see Section 2. What is more, it can be shown that the solution is in fact smooth up to the blowup time (for example by considering the time evolution at a higher regularity as in [10], see also the discussion in Section 1.2).

Finally, we note that the methods used in this paper work in principle in $H^{s} \times H^{s-1}$, with $s=s_{c}+\varepsilon, \varepsilon>0$. To obtain a similar result at the critical regularity, Strichartz estimates as in [15] would be required. 
2. Extension to other space dimensions. Large parts of the proof can be generalized to other space dimensions with obvious adjustments. The key problem is the spectral analysis for the operator corresponding to the linearization around $u_{T, x_{0}, a}^{*}$. In seven dimensions, we can exploit some special structural properties that simplify the problem, see Section 1.2 for a more detailed discussion. However, we expect that a result analogous to Theorem $1.1 \mathrm{can}$ be established in all space dimensions $d \geq 5$.

1.1. Discussion and known results. The overall strategy of the proof of Theorem 1.1 is based on the approach developed by Donninger and the second author in [18] to prove, without symmetry assumptions, the nonlinear asymptotic stability of the ODE blowup solution for the focusing supercritical wave equation in three space dimensions. This has recently been generalized to Eq. (1.1) in $d=5$ by Donninger and Chatzikaleas [10]. However, we stress that, as opposed to [18] and [10], the underlying spectral problem in this paper is much more difficult. Nevertheless, by building on ideas from [13] and [12], we develop a new and efficient method to treat such problems, see Sec. 4.

We also refer to Donninger and Zenginoğlu [20] as well as to Donninger and Burtscher [8], where techniques similar to [18] have been used to investigate non-scattering, global solutions to Eq. (1.1) in three-dimensions, where the problem is subcritical and conformally invariant. In this case much more is known in fact, see the work of Merle and Zaag, e.g. [35]. In the subcritical case, the existence of self-similar blowup solutions to Eq. (1.1) has been established by Bizon, Breitenlohner, Maison and Wasserman [4], but these profiles cease to be smooth outside a backward lightcone.

In the energy critical dimension $d=4$, non-trivial self-similar solutions can be excluded. Instead, non self-similar blowup (type II) occurs, see the corresponding construction by Hillairet and Raphaël [25]. In the supercritical case, $d \geq 5$, a similar approach as in [4] can be used to show the existence of infinitely many non-trivial self-similar blow up solutions.

To the best of our knowledge, no such solution has ever been found in closed form for the focusing nonlinear wave equation and $u^{*}$ is the first example of this type. Finally, in higher space dimensions the result of Collot [11] on the existence of (at least co-dimension two unstable) non self-similar blowup solutions applies to Eq. (1.1), which makes the picture even more complex.

To investigate the role of self-similar blowup solutions in the generic time evolution, numerical investigations have been performed by Bizoń, Chmaj and Tabor [6] in the three-dimensional radial setting. Their findings suggest the following picture: For small data, the solution disperses, while for large, generic data the solution blows up in a self-similar manner via the ODE profile. For data fine-tuned to the threshold between these two basins of attraction, the evolution is described by a non-trivial self-similar solution which is approached for some intermediate period before one of the two above scenarios occurs. We note that a similar phenomenology has been observed in numerical experiments for supercritical wave maps [5], [3]. The interest in performing these numerical studies stems from the desire of the authors to understand critical phenomena in gravitational collapse, where threshold solutions are typically self-similar [24]. Furthermore, they propose supercritical focusing wave equations and wave maps as toy models.

From an analytic point of view however, the description of threshold phenomena for supercritical wave equations in terms of self-similar solutions is completely open and Theorem 1.1 
is the first step in this direction. In fact, we strongly believe that $u^{*}$ is a threshold solution for the supercritical cubic wave equation in the sense of [6].

Finally, we note that in the energy critical case much more is known. There, the threshold for blowup is described in terms of the Talenti-Aubin solution $W$, see the seminal work by Kenig and Merle [27]. Our main result is somehow in the spirit of the work by Krieger and Schlag [29], where a co-dimension one stability result was obtained for the family $\left\{W_{\lambda}\right\}$ of rescalings of $W$ in a topology stronger than the energy (in the radial setting). The threshold character of the constructed manifold was then described by Krieger, Nakanishi and Schlag in [32]. Their work was inspired by previous numerical observations made by Bizoń, Chmaj and Tabor in [6]. Later on, in a series of papers [31], [30], [33], Krieger, Nakanishi and Schlag finally gave a fairly complete characterization of the threshold dynamics in the energy space around a co-dimension one manifold containing also type II blowup solutions. In this context, we also refer to the recent papers by Krieger [28], respectively, by Burzio and Krieger [9]. In the supercritical case, we are only at the very beginning of understanding these types of phenomena. In particular, a description of threshold phenomena as detailed as in the critical case seems completely out of reach at the moment.

We conclude the discussion with some general comments. Obviously, the blowup profile is defined on all of $\mathbb{R}^{d}$ and it would be interesting to investigate its stability properties on the whole space, see the recent paper by Biernat, Donninger and the second author [2]. Furthermore, $u^{*}$ as defined in Eq. (1.3) is a global smooth solution to Eq. (1.1) for all $t \geq 1$. Its self-similar character implies that it has a time-independent critical norm. In fact $\left\|\left(u^{*}(t, \cdot), \partial_{t} u^{*}(t, \cdot)\right)\right\|_{\dot{H}^{s_{c} \times \dot{H}^{s_{c}-1}\left(\mathbb{R}^{d}\right)}}=\infty$, which is in agreement with the characterization of global, scattering solutions obtained by Dodson and Lawrie in [14].

1.2. Overview of the paper. In the following, we give a brief overview of the main steps in the proof of Theorem 1.1 highlighting the differences to previous works.

In Section 2, we reformulate Eq. (1.1) as a first order system in similarity variables, $(t, x) \in$ $\bigcup_{t \in[0, T)}\{t\} \times \overline{\mathbb{B}_{T-t}^{7}} \rightarrow(\tau, \xi) \in[0, \infty) \times \overline{\mathbb{B}^{7}}$, which transforms $u_{T, x_{0}, a}^{*}$ into a 7 -parameter family of static solutions $\Psi_{a}^{*}, a \in \mathbb{R}^{7}$, of the abstract equation

$$
\partial_{\tau} \Psi(\tau)=\mathbf{L} \Psi(\tau)+\mathbf{N}(\Psi(\tau))
$$

where $\mathbf{L}$ represents the transformed linear wave operator. We insert the modulation ansatz $\Psi(\tau)=\Psi_{a(\tau)}^{*}+\Phi(\tau)$ and by assuming that $a(\tau) \rightarrow a_{\infty}$ as $\tau \rightarrow \infty$, we write the resulting equation for $\Phi$ as

$$
\partial_{\tau} \Phi(\tau)=\left[\mathbf{L}+\mathbf{L}_{a_{\infty}}^{\prime}\right] \Phi(\tau)+\mathbf{G}_{a(\tau)}(\Phi(\tau))-\partial_{\tau} \Psi_{a(\tau)}^{*}
$$

with $\mathbf{L}_{a_{\infty}}^{\prime}$ containing a potential term and $\mathbf{G}_{a(\tau)}$, the remaining nonlinearity.

Sections 3 - 5 are devoted to the analysis of operators of the type $\mathbf{L}_{a}=\mathbf{L}+\mathbf{L}_{a}^{\prime}$ for sufficiently small $a$. They are defined as unbounded operators $\mathbf{L}_{a}: \mathcal{D}\left(\mathbf{L}_{a}\right) \subset \mathcal{H} \rightarrow \mathcal{H}$, where $\mathcal{H}=$ $H^{k}\left(\mathbb{B}^{7}\right) \times H^{k-1}\left(\mathbb{B}^{7}\right)$. Concerning the topology, it is important to work strictly above scaling and we take the first integer value $k>s_{c}$, i.e., $k=3$. This is in contrast to [18], [10], where the regularity was chosen such that $k>\frac{d}{2}$, which then allows the usage of an $L^{\infty}$ embedding to estimate the nonlinearity. This however comes at a price: in order to prove that $\mathbf{L}_{a}$ 
generates a semigroup, an equivalent inner product which involves a very special (and kind of magical) boundary term has to be used. This term becomes more and more complicated in higher space dimensions, see [10]. In this paper, we work at a lower regularity, which considerably simplifies the equivalent inner product that is used for the semigroup argument (see Section 3) but still allows the usage of Sobolev embedding for the nonlinear estimates, see Lemma 6.1.

In Section 4 and Section 5 we analyze the spectrum of $\mathbf{L}_{a}$ and prove suitable growth bounds for the associated semigroup $\mathbf{S}_{a}(\tau)$. While in [18], [10] the spectral problem associated to the linearization around the trivial ODE profile could easily be solved by elementary methods, we are facing new challenges here. Since $u_{T}^{*}$ has a non-trivial spatial profile we obtain a non-trivial potential in the linearization. This severly complicates the problem. Furthermore, in addition to the eigenvalues $\lambda=0$ and $\lambda=1$ which are induced by the Lorentz symmetry and the space-time translation invariance of Eq. (1.1) there is another genuinely unstable eigenvalue $\lambda^{*}$. The symmetry eigenvalues $\lambda=0$ and $\lambda=1$ are the same in all space dimensions and their geometric multiplicity is equal to $d$, respectively to $d+1$ (note that this is different from [18], [10], where no spatial translations had to be taken into account). For $\lambda^{*}$, on the other hand, numerics indicate that it is real ${ }^{1}$ and decreases as the dimension $d$ grows. In fact, it appears that $\lambda^{*}(d) \rightarrow 2$ as $d \rightarrow \infty$. As it turns out, $\lambda^{*}=3$ in $d=7$, and the corresponding eigenfunction is explicit. For $a=0$, the potential is radial and a decomposition into spherical harmonics reduces the eigenvalue problem to a family of non-trivial ODEs. For $d=7$, we are able to analyze these equations by improving the techniques developed by the first author together with Costin and Donninger [12], see also [13]. The case $a \neq 0$ is then treated perturbatively analogous to [18]. More precisely, we show that there is an $\tilde{\omega}>0$ such that for the spectrum of $\mathbf{L}_{a}$ we have

$$
\sigma\left(\mathbf{L}_{a}\right) \subset\{\lambda \in \mathbb{C}: \operatorname{Re} \lambda \leq-\tilde{\omega}\} \cup\{0,1,3\}
$$

for all $a \in \mathbb{R}^{7}$ that are sufficiently small. It has to be investigated to which extent this approach can be applied to other space dimensions.

Based on the information on the spectrum of $\mathbf{L}_{a}$ and suitable bounds on the resolvent, we conclude Section 5 with estimates for the semigroup $\mathbf{S}_{a}(\tau)$ that guarantee exponetial decay 'transversal' to the unstable directions. Another indispensable ingredient of the proof of the main result are Lipschitz estimates with respect to $a$, for basically all relevant quantities that occur at the linear (and later at the nonlinear) level.

In Section 6 we turn to the analysis of Eq. (1.10). In fact, we study the associated integral equation

$$
\Phi(\tau)=\mathbf{S}_{a_{\infty}}(\tau) \mathbf{u}+\int_{0}^{\tau} \mathbf{S}_{a_{\infty}}(\tau-\sigma)\left[\mathbf{G}_{a(\sigma)}(\Phi(\sigma))-\partial_{\sigma} \Psi_{a(\sigma)}^{*}\right] d \sigma
$$

for $\mathbf{u} \in \mathcal{H}$. The function spaces for $\Phi$ and $a$ are chosen in a way that reflects their desired properties, i.e., that $\Phi(\tau)$ decays exponentially and that $a(\tau) \rightarrow a_{\infty}$ as $\tau \rightarrow \infty$. In Section 6.2 we proceed along the lines of [18]: First, we derive a modulation equation for $a(\tau)$ and show that $a(\tau)$ can be chosen such that the instability induced by the Lorentz transform can be controlled. Then, we modify the initial data by subtracting suitable elements of the

\footnotetext{
${ }^{1}$ Note that, due to non-selfadjoint character of the underlying spectral problem, eigenvalues are not necessarily real.
} 
subspaces corresponding to the remaining instabilities to obtain a solution $\Phi$ to Eq. (1.11), with $\mathbf{u}$ replaced by

$$
\mathbf{u}-\mathbf{C}(\Phi, a, \mathbf{u})
$$

provided that $\mathbf{u}$ and $a$ are sufficiently small. Here, $\mathbf{C}=\mathbf{C}_{1}+\mathbf{C}_{2}$ serves to suppress the instabilities originating from $\lambda=1$ and $\lambda^{*}=3$. The final argument contained in Section 6.3 is different from previous works, in particular, the Lipschitz dependence of the blowup parameters was not addressed so far (in addition to the fact that we have a co-dimension one condition here). In order to remove the correction, we consider initial data for Eq. (1.1) of the form

$$
u[0]=u_{1,0,0}^{*}[0]+\mathbf{v}+\alpha \mathbf{h},
$$

for $\alpha \in \mathbb{R}$, which transforms into initial data

$$
\Phi(0)=\mathbf{U}\left(\mathbf{v}+\alpha \mathbf{h}, T, x_{0}\right)
$$

for Eq. (1.10). We prove suitable Lipschitz estimates for $\mathbf{U}$ (here the additional regularity assumption on $\mathbf{v}$ comes into play) and apply a fixed point argument to show that for every suitably small $\mathbf{v} \in \mathcal{Y}$, there are parameters $\left(a, T, x_{0}, \alpha\right)$ close to $(0,1,0,0)$ depending Lipschitz continuously on $\mathbf{v}$ such that

$$
\mathbf{C}\left(\Phi, a, \mathbf{U}\left(\mathbf{v}+\alpha \mathbf{h}, T, x_{0}\right)\right)=0 .
$$

In particular, there exists a solution $\Phi$ satisfying

$$
\Phi(\tau)=\mathbf{S}_{a_{\infty}}(\tau) \mathbf{U}\left(\mathbf{v}+\alpha \mathbf{h}, T, x_{0}\right)+\int_{0}^{\tau} \mathbf{S}_{a_{\infty}}(\tau-\sigma)\left[\mathbf{G}_{a(\sigma)}(\Phi(\sigma))-\partial_{\sigma} \Psi_{a(\sigma)}^{*}\right] d \sigma
$$

with $\|\Phi(\tau)\|_{H^{3} \times H^{2}\left(\mathbb{B}^{7}\right)} \lesssim e^{-\omega \tau}$ for all $\tau \geq 0$ and some $\omega>0$. By reverting to the original coordinates, this yields the main result.

1.3. Notation and Conventions. Throughout the whole paper the Einstein summation convention is in force, i.e., we sum over repeated upper and lower indices, where latin indices run from $1, \ldots, d$.

We write $\mathbb{N}$ for the natural numbers $\{1,2,3, \ldots\}, \mathbb{N}_{0}:=\{0\} \cup \mathbb{N}$. Furthermore, $\mathbb{R}^{+}:=\{x \in$ $\mathbb{R}: x>0\}$. Also, $\overline{\mathbb{H}}$ stands for the closed complex right half-plane. By $\mathbb{B}_{R}^{d}\left(x_{0}\right)$ we denote the open ball of radius $R>0$ in $\mathbb{R}^{d}$ centered at $x_{0} \in \mathbb{R}^{d}$. The unit ball is abbreviated by $\mathbb{B}^{d}:=\mathbb{B}_{1}^{d}(0)$ and $\mathbb{S}^{d-1}:=\partial \mathbb{B}^{d}$.

By $L^{2}\left(\mathbb{B}_{R}^{d}\left(x_{0}\right)\right)$ and $H^{k}\left(\mathbb{B}_{R}^{d}\left(x_{0}\right)\right), k \in \mathbb{N}_{0}$, we denote the Lebesgue and Sobolev spaces obtained from the completion of $C^{\infty}\left(\mathbb{B}_{R}^{d}\left(x_{0}\right)\right)$ with respect to the usual norm

$$
\|u\|_{H^{k}\left(\mathbb{B}_{R}^{d}\left(x_{0}\right)\right)}^{2}:=\sum_{|\alpha| \leq k}\left\|\partial^{\alpha} u\right\|_{L^{2}\left(\mathbb{B}_{R}^{d}\left(x_{0}\right)\right)}^{2},
$$

with $\alpha \in \mathbb{N}_{0}^{d}$ denoting a multi-index and $\partial^{\alpha} u=\partial_{1}^{\alpha_{1}} \ldots \partial_{d}^{\alpha_{d}} u$, where $\partial_{i} u(x)=\partial_{x_{j}} u(x)$. For vector-valued functions, we use boldface letters, e.g., $\mathbf{f}=\left(f_{1}, f_{2}\right)$ and we sometime write $[\mathbf{f}]_{1}:=f_{1}$ to extract a single component. Throughout the paper, $W(f, g)$ denotes the Wronskian of two functions $f, g \in C^{1}(I), I \subset \mathbb{R}$, where we use the convention $W(f, g)=$ $f g^{\prime}-f^{\prime} g$ with $f^{\prime}$ denoting the first derivative.

On a Hilbert space $\mathcal{H}$ we denote by $\mathcal{B}(\mathcal{H})$ the set of bounded linear operators. For a closed linear operator $(L, \mathcal{D}(L))$ on $\mathcal{H}$, we define the resolvent set $\rho(L)$ as the set of all $\lambda \in \mathbb{C}$ such that $R_{L}(\lambda):=(\lambda-L)^{-1}$ exists as a bounded operator on the whole underlying space. Furthermore, the spectrum of $L$ is defined as $\sigma(L):=\mathbb{C} \backslash \rho(L)$ and the point spectrum is 
denoted by $\sigma_{p}(L) \subset \sigma(L)$. The notation $a \lesssim b$ means $a \leq C b$ for an absolute constant $C>0$ and we write $a \simeq b$ if $a \lesssim b$ and $b \lesssim a$. If $a \leq C_{\varepsilon} b$ for a constant $C_{\varepsilon}>0$ depending on some parameter $\varepsilon$, we write $a \lesssim_{\varepsilon} b$.

Spherical harmonics. For fixed $d \geq 3$ we denote by $Y_{\ell}: \mathbb{S}^{d-1} \rightarrow \mathbb{C}$ a spherical harmonic function of degree $\ell \in \mathbb{N}_{0}$ (i.e., the restriction of a harmonic homogeneous polynomial $H_{\ell}\left(x_{1}, \ldots, x_{d}\right)$ of degree $\ell$ in $\mathbb{R}^{d}$ to the $(d-1)$-sphere). In particular, $Y_{\ell}$ is an eigenfunction for the Laplace-Beltrami operator on $\mathbb{S}^{d-1}$ with eigenvalue $\ell(\ell+d-2)$ and

$$
\int_{\mathbb{S}^{d}} Y_{\ell}(\omega) \overline{Y_{\ell^{\prime}}(\omega)} d \sigma(\omega)=\delta_{\ell \ell^{\prime}}
$$

For each $\ell \in \mathbb{N}$, we denote by $M_{d, \ell} \in \mathbb{N}$ the number of linearly independent spherical harmonics, and we designate by $\left\{Y_{\ell, m}: m \in \Omega_{\ell}\right\}, \Omega_{\ell}=\left\{1, \ldots, M_{d, \ell}\right\}$ a linearly independent orthonormal set such that

$$
\int_{\mathbb{S}^{d}} Y_{\ell, m}(\omega) \overline{Y_{\ell, m^{\prime}}(\omega)} d \sigma(\omega)=\delta_{m m^{\prime}}
$$

Obviously, one has $\Omega_{0}=\{1\}, \Omega_{1}=\{1, \ldots, d\}$, and $Y_{0,1}(\omega)=c_{1}, Y_{1, m}(\omega)=\tilde{c}_{m} \omega_{m}$ for suitable normalization constants $c_{1}, \tilde{c}_{m} \in \mathbb{R}$. For $g \in C^{\infty}\left(\mathbb{S}^{d-1}\right)$, we define $\mathcal{P}_{\ell}: L^{2}\left(\mathbb{S}^{d-1}\right) \rightarrow L^{2}\left(\mathbb{S}^{d-1}\right)$ by

$$
\mathcal{P}_{\ell} g(\omega):=\sum_{m \in \Omega_{\ell}}\left(g \mid Y_{\ell, m}\right)_{L^{2}\left(\mathbb{S}^{d-1}\right)} Y_{\ell, m}(\omega)
$$

It is well-known, see e.g. [1], that $\mathcal{P}_{\ell}$ defines a self-adjoint projection on $L^{2}\left(\mathbb{S}^{d-1}\right)$ and that $\lim _{n \rightarrow \infty}\left\|g-\sum_{\ell=0}^{n} \mathcal{P}_{\ell} g\right\|_{L^{2}\left(\mathbb{S}^{d-1}\right)}=0$. This can be extended to Sobolev spaces, in particular, $\lim _{n \rightarrow \infty}\left\|g-\sum_{\ell=0}^{n} \mathcal{P}_{\ell} g\right\|_{H^{k}\left(\mathbb{S}^{d-1}\right)} \rightarrow 0$ for all $g \in C^{\infty}\left(\mathbb{S}^{d-1}\right)$, see e.g. [18], Lemma A.1. Furthermore, for $f \in C^{\infty}\left(\overline{\mathbb{B}^{d}}\right)$ and

$$
\left[P_{\ell} f\right](x):=\sum_{m \in \Omega_{\ell}}\left(f(|x| \cdot) \mid Y_{\ell, m}\right)_{L^{2}\left(\mathbb{S}^{d-1}\right)} Y_{\ell, m}\left(\frac{x}{|x|}\right)
$$

we have, see for example Lemma A.2,

$$
\lim _{n \rightarrow \infty}\left\|f-\sum_{\ell=0}^{n} P_{\ell} f\right\|_{H^{k}\left(\mathbb{B}^{d}\right)} \rightarrow 0 .
$$

\section{DynAmiCAl SYSTEM FORMUlation in ADAPTED COORDinAtes}

We consider small perturbations of the blowup initial data $u_{1,0,0}^{*}[0]=\left(u_{1,0,0}^{*}(0, \cdot), \partial_{t} u_{1,0,0}^{*}(0, \cdot)\right)$,

$$
u_{1,0,0}^{*}(0, x)=\psi_{0}^{*}(x)=U(|x|),\left.\quad \partial_{t} u_{1,0,0}^{*}(t, x)\right|_{t=0}=\psi_{0}^{*}(x)+x^{j} \partial_{j} \psi_{0}^{*}(x)=U(|x|)+|x| U^{\prime}(|x|),
$$

i.e., we study the initial value problem for Eq. (1.1) with data of the form

$$
u[0]=u_{1,0,0}^{*}[0]+(f, g) .
$$

Our aim is to identify a suitable class of functions $(f, g)$ such that the corresponding solution blows up in forward time $T>0$ at some point $x_{0} \in \mathbb{R}^{7}$ and converges to $u_{T, x_{0}, a}^{*}$ for some 
$a \in \mathbb{R}^{7}$ as $t \rightarrow T$. In general, the blowup parameters $\left(T, x_{0}, a\right)$ will depend on the data. Thus, for (yet unknown) parameters $T>0$ and $x_{0} \in \mathbb{R}^{7}$ we define adapted coordinates

$$
\tau=-\log (T-t)+\log T, \quad \xi=\frac{x-x_{0}}{T-t},
$$

and we set

$$
\psi(\tau, \xi)=T e^{-\tau} u\left(T-T e^{-\tau}, T e^{-\tau} \xi+x_{0}\right)
$$

such that Eq. (1.1) reads

$$
\left[\partial_{\tau}^{2}+3 \partial_{\tau}+2 \xi^{j} \partial_{\xi^{j}} \partial_{\tau}-\left(\delta^{j k}-\xi^{j} \xi^{k}\right) \partial_{\xi^{j}} \partial_{\xi^{k}}+4 \xi^{j} \partial_{\xi^{j}}+2\right] \psi(\tau, \xi)=\psi(\tau, \xi)^{3} .
$$

Now, for each $a \in \mathbb{R}^{7}, \psi_{a}^{*}$ defined in Eq. (1.6) is a static solution to Eq. (2.2). As mentioned above, there is a $\delta^{*}>0$ such that $\psi_{a}^{*} \in C^{\infty}\left(\mathbb{R}^{7}\right)$ for all $a \in \overline{\mathbb{B}_{\delta^{*}}^{7}}$. Furthermore, $\psi_{a}^{*}$ depends smoothly on $a$ and by the fundamental theorem of calculus,

$$
\psi_{a}^{*}(\xi)-\psi_{b}^{*}(\xi)=\left(a^{j}-b^{j}\right) \int_{0}^{1} \partial_{\alpha_{j}} \psi_{\alpha(s)}^{*}(\xi) d s,
$$

for $\alpha(s)=b+s(a-b)$. This implies the Lipschitz estimate

$$
\left\|\psi_{a}^{*}-\psi_{b}^{*}\right\|_{\dot{H}^{k}\left(\mathbb{B}^{7}\right)} \lesssim_{k}|a-b|
$$

for all $a, b, \in \mathbb{B}_{\delta^{*}}^{7}$ and $k \in \mathbb{N}_{0}$.

We write Eq. (2.2) as a first order system by setting

$$
\psi_{1}(\tau, \xi):=\psi(\tau, \xi), \quad \psi_{2}(\tau, \xi):=\partial_{\tau} \psi(\tau, \xi)+\xi^{j} \partial_{j} \psi(\tau, \xi)+\psi(\tau, \xi)
$$

and $\Psi(\tau)=\left(\psi_{1}(\tau, \cdot), \psi_{2}(\tau, \cdot)\right)$, which yields

$$
\partial_{\tau} \Psi(\tau)=\tilde{\mathbf{L}} \Psi(\tau)+\mathbf{N}(\Psi(\tau))
$$

with

$$
\tilde{\mathbf{L}} \mathbf{u}(\xi):=\left(\begin{array}{c}
-\xi^{j} \partial_{j} u_{1}(\xi)-u_{1}(\xi)+u_{2}(\xi) \\
\partial_{j} \partial^{j} u_{1}(\xi)-\xi^{j} \partial_{j} u_{2}(\xi)-2 u_{2}(\xi)
\end{array}\right)
$$

and $\mathbf{N}(\mathbf{u})=\left(0, u_{1}^{3}\right)$. Eq. (2.5) has the explicit solution $\Psi_{a}^{*}=\left(\psi_{1, a}^{*}, \psi_{2, a}^{*}\right)$ whereby $\psi_{1, a}^{*}=\psi_{a}^{*}$ and $\psi_{2, a}^{*}(\xi)=\xi^{j} \partial_{j} \psi_{a}^{*}(\xi)+\psi_{a}^{*}(\xi)$. In the following, we assume that $\Psi(\tau)=\Psi_{a}^{*}+\Phi(\tau)$, where $\Phi$ is a small perturbation. The parameter $a \in \mathbb{R}^{7}$ depends on the initial data in general. We therefore insert the modulation ansatz

$$
\Psi(\tau)=\Psi_{a(\tau)}^{*}+\Phi(\tau)
$$

into Eq. (2.5), where we assume that $a(0)=0$ and $\lim _{\tau \rightarrow \infty} a(\tau)=a_{\infty} \in \mathbb{R}^{7}$. We obtain

$$
\partial_{\tau} \Phi(\tau)=\left[\tilde{\mathbf{L}}+\mathbf{L}_{a(\tau)}^{\prime}\right] \Phi(\tau)+\mathbf{F}_{a(\tau)}(\Phi(\tau))-\partial_{\tau} \Psi_{a(\tau)}^{*}
$$

with

$$
\mathbf{F}_{a(\tau)}(\mathbf{u})=\left(\begin{array}{c}
0 \\
u_{1}^{3}+3 \psi_{a(\tau)}^{*} u_{1}^{2}
\end{array}\right), \quad \mathbf{L}_{a}^{\prime} \mathbf{u}=\left(\begin{array}{c}
0 \\
V_{a} u_{1}
\end{array}\right), \quad V_{a}(\xi):=3 \psi_{a}^{*}(\xi)^{2} .
$$

Analogous to Eq. (2.3) one can show that for $k \in \mathbb{N}_{0}$,

$$
\left\|V_{a}-V_{b}\right\|_{\dot{H}^{k}\left(\mathbb{B}^{7}\right)} \lesssim_{k}|a-b|
$$


for all sufficiently small $a, b \in \mathbb{R}^{7}$. We equip Eq. (2.6) with initial data

$$
\Phi(0)=\mathbf{U}\left((f, g), T, x_{0}\right)=\mathcal{R}\left((f, g), T, x_{0}\right)+\mathcal{R}\left(\Psi_{0}^{*}, T, x_{0}\right)-\Psi_{0}^{*}
$$

where

$$
\mathcal{R}\left((f, g), T, x_{0}\right):=\left(\begin{array}{c}
T f\left(T \cdot+x_{0}\right) \\
T^{2} g\left(T \cdot+x_{0}\right)
\end{array}\right),
$$

which are obtained by transforming Eq. (2.1) accordingly. To deal with the time-dependent potential in Eq. (2.6) we rewrite that problem as

$$
\begin{aligned}
\partial_{\tau} \Phi(\tau) & =\left[\tilde{\mathbf{L}}+\mathbf{L}_{a_{\infty}}^{\prime}\right] \Phi(\tau)+\mathbf{G}_{a(\tau)}(\Phi(\tau))-\partial_{\tau} \Psi_{a(\tau)}^{*}, \quad \tau>0 \\
\Phi(0) & =\mathbf{U}\left((f, g), T, x_{0}\right)
\end{aligned}
$$

where

$$
\mathbf{G}_{a(\tau)}(\Phi(\tau)):=\left[\mathbf{L}_{a(\tau)}^{\prime}-\mathbf{L}_{a_{\infty}}^{\prime}\right] \Phi(\tau)+\mathbf{F}_{a(\tau)}(\Phi(\tau))
$$

We study Eq. (2.9) as an abstract initial value problem on $\mathcal{H}=H^{3}\left(\mathbb{B}^{7}\right) \times H^{2}\left(\mathbb{B}^{7}\right)$ equipped with the usual norm

$$
\|\mathbf{u}\|:=\left\|\left(u_{1}, u_{2}\right)\right\|_{H^{3} \times H^{2}\left(\mathbb{B}^{7}\right)} .
$$

We show that for suitably small functions $\mathbf{v}=\left(v_{1}, v_{2}\right)$, there is an $\alpha \in \mathbb{R}$ as well as parameters $\left(T, x_{0}, a\right)$ depending on $\mathbf{v}$ such that for $(f, g)=\mathbf{v}+\alpha \mathbf{h}$ and $\mathbf{h}$ defined in Eq. (1.8) we obtain a unique solution $\Phi(\tau)$ to Eq. (2.9) that decays to zero as $\tau \rightarrow \infty$.

\section{SEMigroup THEORY}

3.1. The free time evolution. We consider the differential operator $\tilde{\mathbf{L}}: \mathcal{D}(\tilde{\mathbf{L}}) \subset \mathcal{H} \rightarrow \mathcal{H}$ for $\mathcal{D}(\tilde{\mathbf{L}})=C^{4}\left(\overline{\mathbb{B}^{7}}\right) \times C^{3}\left(\overline{\mathbb{B}^{7}}\right)$ such that $\tilde{\mathbf{L}}$ is densely defined. In the following, we prove that the closure of $\tilde{\mathbf{L}}$ is the generator of a strongly-continuous semigroup. For this, we introduce another norm on our function space.

Equivalent norm on $H^{3} \times H^{2}\left(\mathbb{B}^{7}\right)$. On $C^{3}\left(\overline{\mathbb{B}^{7}}\right) \times C^{2}\left(\overline{\mathbb{B}^{7}}\right)$ we define

$$
\begin{aligned}
(\mathbf{u} \mid \mathbf{v})_{1} & :=\int_{\mathbb{B}^{7}} \partial_{i} \partial_{j} \partial_{k} u_{1}(\xi) \overline{\partial^{i} \partial^{j} \partial^{k} v_{1}(\xi)} d \xi+\int_{\mathbb{B}^{7}} \partial_{i} \partial_{j} u_{2}(\xi) \overline{\partial^{i} \partial^{j} v_{2}(\xi)} d \xi \\
& +\int_{\mathbb{S}^{6}} \partial_{i} \partial_{j} u_{1}(\omega) \overline{\partial^{i} \partial^{j} v_{1}(\omega)} d \sigma(\omega) \\
(\mathbf{u} \mid \mathbf{v})_{2} & :=\int_{\mathbb{B}^{7}} \partial_{i} \Delta u_{1}(\xi) \overline{\partial^{i} \Delta v_{1}(\xi)} d \xi+\int_{\mathbb{B}^{7}} \partial_{i} \partial_{j} u_{2}(\xi) \overline{\partial^{i} \partial^{j} v_{2}}(\xi) d \xi+\int_{\mathbb{S}^{6}} \partial_{i} u_{2}(\omega) \overline{\partial^{i} v_{2}(\omega)} d \sigma(\omega)
\end{aligned}
$$

and set

$$
\begin{aligned}
(\mathbf{u} \mid \mathbf{v})_{\mathcal{H}} & :=4(\mathbf{u} \mid \mathbf{v})_{1}+(\mathbf{u} \mid \mathbf{v})_{2} \\
& +\int_{\mathbb{S}^{6}} \partial_{i} u_{1}(\omega) \overline{\partial^{i} v_{1}(\omega)} d \sigma(\omega)+\int_{\mathbb{S}^{6}} u_{1}(\omega) \overline{v_{1}(\omega)} d \sigma(\omega)+\int_{\mathbb{S}^{6}} u_{2}(\omega) \overline{v_{2}(\omega)} d \sigma(\omega) .
\end{aligned}
$$

Furthermore, we let

$$
\|\mathbf{u}\|_{\mathcal{H}}:=\sqrt{(\mathbf{u} \mid \mathbf{u})_{\mathcal{H}}}
$$

and show that this defines an equivalent norm on $H^{3} \times H^{2}\left(\mathbb{B}^{7}\right)$. 
Lemma 3.1. We have

$$
\|\mathbf{u}\|_{\mathcal{H}} \simeq\|\mathbf{u}\|
$$

for all $\mathbf{u} \in C^{3}\left(\overline{\mathbb{B}^{7}}\right) \times C^{2}\left(\overline{\mathbb{B}^{7}}\right)$. In particular, $\|\cdot\|_{\mathcal{H}}$ defines an equivalent norm on $\mathcal{H}$.

The proof is given in Appendix A.1 and is based on the trace theorem as well as on the fact that

$$
\|u\|_{L^{2}\left(\mathbb{B}^{7}\right)}^{2} \lesssim\|\nabla u\|_{L^{2}\left(\mathbb{B}^{7}\right)}^{2}+\|u\|_{L^{2}\left(\mathbb{S}^{6}\right)}^{2},
$$

for all $u$ sufficiently regular. By an approximation argument, the result extends to all of $H^{3} \times H^{2}\left(\mathbb{B}^{7}\right)$, where the boundary integrals are understood in the sense of traces. This new inner product is tailor-made to derive the following estimate for the operator $\tilde{\mathbf{L}}$.

Lemma 3.2. We have $\operatorname{Re}(\tilde{\mathbf{L}} \mathbf{u} \mid \mathbf{u})_{\mathcal{H}} \leq-\frac{1}{2}\|\mathbf{u}\|_{\mathcal{H}}^{2}$ for all $\mathbf{u} \in \mathcal{D}(\tilde{\mathbf{L}})$.

The lenghty, but straightforward proof is given in Appendix A.2.

Lemma 3.3. Let $\lambda=\frac{5}{2}$. Then

$$
\operatorname{rg}(\lambda-\tilde{\mathbf{L}}) \subset \mathcal{H}
$$

is a dense subset.

Proof. Let $\tilde{\mathbf{f}} \in \mathcal{H}$ and $\varepsilon>0$ be arbitrary. By density, there is an $\mathbf{f} \in C^{\infty}\left(\overline{\mathbb{B}^{7}}\right) \times C^{\infty}\left(\overline{\mathbb{B}^{7}}\right)$ such that $\|\mathbf{f}-\tilde{\mathbf{f}}\|<\frac{\varepsilon}{2}$. For $n \in \mathbb{N}$, define $\mathbf{f}_{n}=\left(f_{1, n}, f_{2, n}\right)$ by

$$
f_{1, n}:=\sum_{\ell=0}^{n} P_{\ell} f_{1}, \quad f_{2, n}:=\sum_{\ell=0}^{n} P_{\ell} f_{2} .
$$

Eq. (1.13) implies that there is an $N \in \mathbb{N}$ such that $\left\|\mathbf{f}_{N}-\mathbf{f}\right\|<\frac{\varepsilon}{2}$. Hence, it suffices to consider the equation $(\lambda-\tilde{\mathbf{L}}) \mathbf{u}=\mathbf{f}_{N}$ and to construct a solution $\mathbf{u} \in \mathcal{D}(\tilde{\mathbf{L}})$. For $u_{2}$, we obtain the equation

$$
u_{2}(\xi)=\xi^{i} \partial_{i} u_{1}(\xi)+(\lambda+1) u_{1}(\xi)-f_{1, N}(\xi)
$$

and $u_{1}$ satisfies the degenerate elliptic problem

$$
-\left(\delta^{i j}-\xi^{i} \xi^{j}\right) \partial_{i} \partial_{j} u_{1}(\xi)+2(\lambda+2) \xi^{i} \partial_{i} u_{1}(\xi)+(\lambda+1)(\lambda+2) u_{1}(\xi)=g_{N}(\xi),
$$

for $g_{N} \in C^{\infty}\left(\overline{\mathbb{B}^{7}}\right)$ given by

$$
g_{N}(\xi)=\xi^{i} \partial_{i} f_{N, 1}(\xi)+(\lambda+2) f_{N, 1}(\xi)+f_{N, 2}(\xi) .
$$

For $\lambda=\frac{5}{2}$, Eq. (3.2) reduces to

$$
-\left(\delta^{i j}-\xi^{i} \xi^{j}\right) \partial_{i} \partial_{j} u_{1}(\xi)+9 \xi^{i} \partial_{i} u_{1}(\xi)+\frac{63}{4} u_{1}(\xi)=g_{N}(\xi)
$$

and by introducing polar coordinates $\rho=|\xi|, \omega=\frac{\xi}{|\xi|}$ the right hand side can be written as

$$
g_{N}(\rho \omega)=\sum_{\ell=0}^{N} \sum_{m \in \Omega_{\ell}} g_{\ell, m}(\rho) Y_{\ell, m}(\omega),
$$

with $g_{\ell, m} \in C^{\infty}[0,1]$. By inserting the ansatz

$$
u_{1}(\rho \omega)=\sum_{\ell=0}^{N} \sum_{m \in \Omega_{\ell}} u_{\ell, m}(\rho) Y_{\ell, m}(\omega),
$$


we obtain a decoupled system of ODEs

$$
\left[-\left(1-\rho^{2}\right) \partial_{\rho}^{2}-\frac{6}{\rho} \partial_{\rho}+9 \rho \partial_{\rho}+\frac{\ell(\ell+5)}{\rho^{2}}+\frac{63}{4}\right] u_{\ell, m}(\rho)=g_{\ell, m}(\rho) .
$$

We set $v_{\ell, m}(\rho):=\rho^{2} u_{\ell, m}(\rho)$ such that Eq. (3.5) transforms into

$$
\left[-\left(1-\rho^{2}\right) \partial_{\rho}^{2}-\frac{2}{\rho} \partial_{\rho}+5 \rho \partial_{\rho}+\frac{(\ell+2)(\ell+3)}{\rho^{2}}+\frac{15}{4}\right] v_{\ell, m}(\rho)=\rho^{2} g_{\ell, m}(\rho),
$$

for $\ell=0, \ldots, N$ and $m \in \Omega_{\ell}$ (however, note that the coefficients only depend on $\ell$ ). This ODE problem can be solved by using hypergeometric functions and has been analyzed in full detail in [20], [18] and [10]. By smoothness of both the coefficients and the right hand side in the interval $(0,1), v_{\ell, m}$ (and hence $\left.u_{\ell, m}\right) \in C^{\infty}(0,1)$. At the two regular singular points $\rho=0$ and $\rho=1$, the Frobenius indices are $\{\ell+2,-3-\ell\}$ and $\left\{0,-\frac{1}{2}\right\}$, respectively. By using a suitable (explicitly known) fundamental system $\left\{\psi_{\ell, 0}, \psi_{\ell, 1}\right\}$ for the homogeneous version of Eq. (3.6) with $\psi_{\ell, 0}, \psi_{\ell, 1}$ being regular at $\rho=0$, respectively at $\rho=1$, a solution to Eq. (3.5) can be written as

$u_{\ell, m}(\rho)=-\frac{\psi_{\ell, 0}(\rho)}{\rho^{2}} \int_{\rho}^{1} \frac{\psi_{\ell, 1}(s)}{W\left(\psi_{\ell, 0}, \psi_{\ell, 1}\right)(s)} \frac{s^{2} g_{\ell, m}(s)}{1-s^{2}} d s-\frac{\psi_{\ell, 1}(\rho)}{\rho^{2}} \int_{0}^{\rho} \frac{\psi_{\ell, 0}(s)}{W\left(\psi_{\ell, 0}, \psi_{\ell, 1}\right)(s)} \frac{s^{2} g_{\ell, m}(s)}{1-s^{2}} d s$,

where $W\left(\psi_{\ell, 0}, \psi_{\ell, 1}\right)(\rho)=C_{\ell}\left(1-\rho^{2}\right)^{-\frac{3}{2}} \rho^{-2}$. In fact, by setting $\ell^{\prime}:=\ell+1 \in\{1, \ldots, N+1\}$ one can immediately use the results obtained in Lemma 4.7 of [10], see Eq. 4.9 there, to infer that $u_{\ell, m} \in C^{\infty}(0,1]$. For the behavior at the origin, one proceeds as in [20]. In particular, by setting $\ell^{\prime \prime}:=\ell+2$ one obtains Eq. 4.8 in [20] with a right hand side $\rho^{2} g_{\ell, m}(\rho)$ instead of $g_{\ell, m}(\rho)$. By studying the asymptotic behavior of $u_{\ell, m}$ and its derivatives near zero, and using the explicit form of $u_{1}$ given in Eq. (3.4) we conclude that $u_{1} \in H^{3}\left(\mathbb{B}^{7}\right) \cap C^{\infty}\left(\overline{\mathbb{B}^{7}} \backslash\{0\}\right)$. Finally, elliptic regularity implies that $u_{1} \in C^{\infty}\left(\overline{\mathbb{B}^{7}}\right)$. With $u_{2}$ defined according to Eq. (3.1), we see that $\mathbf{u} \in \mathcal{D}(\tilde{\mathbf{L}})$, which implies the claim.

The free time-evolution. In view of Lemma 3.2, Lemma 3.3, the Lumer-Phillips Theorem [22], p. 83, Theorem 3.15 and the equivalence of norms, we obtain the following result for the free time-evolution.

Proposition 3.4. The operator $\tilde{\mathbf{L}}: \mathcal{D}(\tilde{\mathbf{L}}) \subset \mathcal{H} \rightarrow \mathcal{H}$ is closable and its closure $(\mathbf{L}, \mathcal{D}(\mathbf{L}))$ generates a strongly-continuous one-parameter semigroup $\mathbf{S}:[0, \infty) \rightarrow \mathcal{B}(\mathcal{H})$ satisfying

$$
\|\mathbf{S}(\tau)\| \leq M e^{-\frac{1}{2} \tau}
$$

for all $\tau \geq 0$ and some constant $M>1$. This implies that the spectrum of $\mathbf{L}$ is contained in a left half-plane,

$$
\sigma(\mathbf{L}) \subset\left\{\lambda \in \mathbb{C}: \operatorname{Re} \lambda \leq-\frac{1}{2}\right\}
$$

and the resolvent is bounded by

$$
\left\|\mathbf{R}_{\mathbf{L}}(\lambda)\right\| \leq \frac{M}{\operatorname{Re} \lambda+\frac{1}{2}}
$$

for all $\lambda \in \mathbb{C}$ with $\operatorname{Re} \lambda>-\frac{1}{2}$.

For the last two statements see [22], p. 55, Theorem 1.10. 
3.2. Perturbations of the free evolution. In the following, we define $\mathbf{L}_{a}^{\prime}$ according to Eq. (2.7). We fix $\delta^{*}>0$ such that $\psi_{a}^{*}$ is smooth for all $a \in \overline{\mathbb{B}_{\delta^{*}}^{7}}$. For the rest of the paper, we assume that $0<\delta<\delta^{*}$.

Lemma 3.5. Let $\delta>0$ be sufficiently small, then for every $a \in \overline{\mathbb{B}_{\delta}^{7}}$ the operator $\mathbf{L}_{a}^{\prime}: \mathcal{H} \rightarrow \mathcal{H}$ is compact. Furthermore, the family of operators $\mathbf{L}_{a}^{\prime} s$ uniformly bounded with respect to $a \in \overline{\mathbb{B}_{\delta}^{7}}$ and Lipschitz continuous, i.e., there is a $K>0$ such that

$$
\left\|\mathbf{L}_{a}^{\prime}-\mathbf{L}_{b}^{\prime}\right\| \leq K|a-b|
$$

for all $a, b \in \overline{\mathbb{B}_{\delta}^{7}}$.

Proof. The uniform boundedness of $\mathbf{L}_{a}^{\prime}$ follows from the smoothness of the potential $V_{a} \in$ $C^{\infty}\left(\overline{\mathbb{B}^{7}}\right)$ with respect to $a$ and the assumption that $a \in \overline{\mathbb{B}_{\delta^{*}}^{7}}$. For the Lipschitz estimate we use that

for all $a, b \in \overline{\mathbb{B}_{\delta}^{7}}$. Hence,

$$
\left\|V_{a}-V_{b}\right\|_{W^{2, \infty}\left(\mathbb{B}^{7}\right)} \lesssim|a-b|
$$

$$
\left\|\left(V_{a}-V_{b}\right) u_{1}\right\|_{H^{2}\left(\mathbb{B}^{7}\right)} \lesssim|a-b|\left\|u_{1}\right\|_{H^{2}\left(\mathbb{B}^{7}\right)} \lesssim|a-b|\left\|u_{1}\right\|_{H^{3}\left(\mathbb{B}^{7}\right)}
$$

for all $\mathbf{u} \in \mathcal{H}$. For fixed $a \in \overline{\mathbb{B}_{\delta}^{7}}$, the compactness of the operator $\mathbf{L}_{a}^{\prime}$ is a consequence of the compact embedding $H^{3}\left(\mathbb{B}^{7}\right) \hookrightarrow H^{2}\left(\mathbb{B}^{7}\right)$.

As a consequence of the Bounded Perturbation Theorem, see [22] p. 158, we obtain the following result.

Corollary 3.6. Let $\delta>0$ be sufficiently small. For any $a \in \overline{\mathbb{B}_{\delta}^{7}}$, the operator

$$
\mathbf{L}_{a}:=\mathbf{L}+\mathbf{L}_{a}^{\prime} . \quad \mathcal{D}\left(\mathbf{L}_{a}\right)=\mathcal{D}(\mathbf{L}) \subset \mathcal{H} \rightarrow \mathcal{H}
$$

generates a strongly-continuous one-parameter semigroup $\mathbf{S}_{a}:[0, \infty) \rightarrow \mathcal{B}(\mathcal{H})$. Furthermore,

$$
\left\|\mathbf{L}_{a}-\mathbf{L}_{b}\right\| \leq K|a-b|
$$

for all $a, b \in \overline{\mathbb{B}_{\delta}^{7}}$ and $K>0$ as in Lemma 3.5

Unfortunately, the growth estimate obtained from this abstract argument is too weak for our purpose. Hence, we analyze the spectrum of the generator $\mathbf{L}_{a}$. First, we consider the case $a=0$. The general case will then be treated perturbatively.

\section{ODE AnALYsis}

For $a=0$, the potential is radial and it will be shown in Section 5.1 that the eigenvalue problem can be reduced to ODE problems by decomposition into spherical harmonics. We define the formal differential operator

$$
\begin{aligned}
\mathcal{T}_{\ell}(\lambda) f(\rho):=\left(1-\rho^{2}\right) f^{\prime \prime}(\rho)+ & {\left[\frac{6}{\rho}-2(\lambda+2) \rho\right] f^{\prime}(\rho) } \\
& -\left[(\lambda+1)(\lambda+2)+\frac{\ell(\ell+5)}{\rho^{2}}-\frac{48}{\left(1+\rho^{2}\right)^{2}}\right] f(\rho)
\end{aligned}
$$

It will be made clear in the proof of Proposition 5.3 that it suffices to study solutions that are smooth on $[0,1]$. We are particularly interested in $\lambda \in \mathbb{C}$ with non-negative real part, i.e. for each $\ell \in \mathbb{N}_{0}$, we study the set

$$
\Sigma_{\ell}:=\left\{\lambda \in \mathbb{C}: \operatorname{Re} \lambda \geq 0 \text { and there exist } f_{\ell}(\cdot ; \lambda) \in C^{\infty}[0,1] \text { satisfying } \mathcal{T}_{\ell}(\lambda) f_{\ell}(\cdot ; \lambda)=0\right\} .
$$


The investigation of the structure of $\Sigma_{\ell}$ is based on a refinement of the approach developed in [12]. Since different strategies are required for different values of $\ell$, two results will be proved in the following.

Proposition 4.1. For all $\ell \geq 2, \Sigma_{\ell}=\emptyset$. Furthermore, for $\ell=0, \Sigma_{0}=\{1,3\}$ with unique solutions

$$
f_{0}(\rho ; 1)=\frac{1-\rho^{2}}{\left(1+\rho^{2}\right)^{2}} \quad \text { and } \quad f_{0}(\rho ; 3)=\frac{1}{\left(1+\rho^{2}\right)^{2}} .
$$

Proposition 4.2. For $\ell=1$, we have $\Sigma_{1}=\{0,1\}$ with unique solutions

$$
f_{1}(\rho ; 0)=\frac{3 \rho-\rho^{3}}{\left(1+\rho^{2}\right)^{2}} \quad \text { and } \quad f_{1}(\rho ; 1)=\frac{\rho}{\left(1+\rho^{2}\right)^{2}} .
$$

\subsection{Proof of Proposition 4.1.}

4.1.1. Preliminary transformations. We study solutions of the equation

$$
\begin{aligned}
\left(1-\rho^{2}\right) f^{\prime \prime}(\rho)+ & {\left[\frac{6}{\rho}-2(\lambda+2) \rho\right] f^{\prime}(\rho) } \\
& -\left[(\lambda+1)(\lambda+2)+\frac{\ell(\ell+5)}{\rho^{2}}-\frac{48}{\left(1+\rho^{2}\right)^{2}}\right] f(\rho)=0
\end{aligned}
$$

First, we transform Eq. (4.2) into an isospectral equation ${ }^{2}$ of the Heun type. This is achieved by a change of variables

$$
\rho=\sqrt{\frac{x}{2-x}} \text { and } f(\rho)=x^{\frac{\ell}{2}}(2-x)^{\frac{\lambda+1}{2}} y(x) .
$$

Thereby, Eq. (4.2) is transformed into

$$
\begin{aligned}
y^{\prime \prime}(x)+\left(\frac{2 \ell+7}{2 x}+\frac{\lambda-1}{x-1}+\frac{1}{2(x-2)}\right) y^{\prime}(x) & \\
& +\frac{(\lambda+\ell+7)(\lambda+\ell-3) x-3 \ell^{2}-4(\lambda+3) \ell-(\lambda+13)(\lambda-3)}{4 x(x-1)(x-2)} y(x)=0 .
\end{aligned}
$$

By Frobenius theory, any $y_{\ell, \lambda} \in C^{\infty}[0,1]$ that solves Eq. (4.3) is in fact analytic on $[0,1]$. Furthermore, the Frobenius indices of Eq. (4.3) at $x=0$ are $s_{1}=0$ and $s_{2}=-\ell-\frac{5}{2}$. Therefore, without loss of generality, we can assume that $y_{\ell, \lambda}(x)$ has the following expansion

$$
y_{\ell, \lambda}(x)=\sum_{n=0}^{\infty} a_{n}(\ell, \lambda) x^{n}, \quad a_{0}(\ell, \lambda)=1
$$

near $x=0$. Since the singular points of Eq. (4.3) are $x=0, x=1$ and $x=2, y_{\ell, \lambda}(x)$ fails to be analytic at $x=1$ precisely when the radius of convergence of the series (4.4) is equal to one. We therefore consider the sequence of coefficients $\left\{a_{n}(\ell, \lambda)\right\}_{n \geq 0}$ and aim to show that

$$
\lim _{n \rightarrow \infty} \frac{a_{n+1}(\ell, \lambda)}{a_{n}(\ell, \lambda)}=1,
$$

whenever the ratio inside the limit is defined for large $n$. To that end, we first derive the recurrence relation for coefficients $a_{n}$. Namely, by putting (4.4) into Eq. (4.2) we obtain

$$
a_{n+2}(\ell, \lambda)=A_{n}(\ell, \lambda) a_{n+1}(\ell, \lambda)+B_{n}(\ell, \lambda) a_{n}(\ell, \lambda),
$$

\footnotetext{
${ }^{2}$ In a sense that the set of values of $\lambda$ that yield $C^{\infty}[0,1]$ solutions is the same for both equations.
} 
where

$$
\begin{gathered}
A_{n}(\ell, \lambda)=\frac{12 n^{2}+4(3 \ell+2 \lambda+12) n+\lambda^{2}+2(2 \ell+9) \lambda+3\left(\ell^{2}+8 \ell-1\right)}{4(2 n+2 \ell+9)(n+2)}, \\
B_{n}(\ell, \lambda)=-\frac{(\ell+\lambda+2 n+7)(\ell+\lambda+2 n-3)}{4(2 n+2 \ell+9)(n+2)},
\end{gathered}
$$

$a_{-1}(\ell, \lambda)=0$ and $a_{0}(\ell, \lambda)=1$. For every nonnegative integer $n$ we define

$$
r_{n}(\ell, \lambda):=\frac{a_{n+1}(\ell, \lambda)}{a_{n}(\ell, \lambda)} .
$$

Since $\lim _{n \rightarrow \infty} A_{n}(\ell, \lambda)=\frac{3}{2}$ and $\lim _{n \rightarrow \infty} B_{n}(\ell, \lambda)=-\frac{1}{2}$, the characteristic equation of Eq. (4.5) is

$$
t^{2}-\frac{3}{2} t+\frac{1}{2}=0
$$

Furthermore, $t_{1}=\frac{1}{2}$ and $t_{2}=1$ are the solutions to Eq. (4.6) and by Poincaré's theorem from the theory of difference equations (see, for example, [21], p. 343) we conclude that given $\lambda \in \overline{\mathbb{H}}$, either $a_{n}(\ell, \lambda)=0$ eventually in $n$ or

$$
\lim _{n \rightarrow \infty} r_{n}(\ell, \lambda)=1
$$

or

$$
\lim _{n \rightarrow \infty} r_{n}(\ell, \lambda)=\frac{1}{2}
$$

We now proceed by separately treating the cases $\ell \geq 2$ and $\ell=0$.

4.1.2. The case $\ell \geq 2$. We claim that $a_{n}(\ell, \lambda)$ does not equal zero eventually in $n$. Indeed, assumptions that $a_{n}(\ell, \lambda)=0$ for large $n$ and $a_{-1}(\ell, \lambda)=0$ imply (by backwards substitution in Eq. (4.5)) that $a_{0}(\ell, \lambda)=0$, which is in contradiction with the assumption that $a_{0}(\ell, \lambda)=1$. Therefore, either (4.7) or (4.8) is true. We claim that Eq. (4.7) holds for all $\lambda \in \overline{\mathbb{H}}$. To establish this, we first derive from Eq. (4.5) the recurrence relation for $r_{n}$. Namely, we have

$$
r_{n+1}(\ell, \lambda)=A_{n}(\ell, \lambda)+\frac{B_{n}(\ell, \lambda)}{r_{n}(\ell, \lambda)}
$$

where

$$
r_{0}(\ell, \lambda)=A_{-1}(\ell, \lambda)=\frac{\lambda^{2}+2(2 \ell+5) \lambda+3\left(\ell^{2}+4 \ell-13\right)}{4(2 \ell+7)} .
$$

Note that $r_{n}(\ell, \lambda)$ can be brought to the form of the ratio of two polynomials in $\lambda$ of degrees that increase with $n$. The explicit form of $r_{n}(\ell, \lambda)$ in fact gets very complicated even for small values of $n$. We therefore desire to construct a "simple" approximate solution $\tilde{r}_{n}$ to the recurrence relation (4.9) which is provably close to $r_{n}$ uniformly in $\ell$ and $\lambda$. Furthermore, proving that a limiting value in $n$ of $\tilde{r}_{n}(\ell, \lambda)$ is 1 will then imply (4.7). The approximation we use is the following

$$
\tilde{r}_{n}(\ell, \lambda)=\frac{\lambda^{2}}{4(n+1)(2 n+2 \ell+7)}+\frac{(4 n+2 \ell+5) \lambda}{2(n+1)(2 n+2 \ell+7)}+\frac{n-1}{n+1}+\frac{3 \ell}{8(n+1)} .
$$

This expression is carefully chosen in order to emulate the behavior of $r_{n}(\ell, \lambda)$ for different values of the participating parameters. Let us elaborate on this. Firstly, notice that $r_{n}(\ell, \lambda)$ is a ratio of two polynomials in $\lambda$ whose degrees differ by two. It is therefore expected that 
$r_{n}(\ell, \lambda)$ behaves like a second degree polynomial for large values of $\lambda$. In fact, this asymptotic behavior can be obtained precisely. Simple induction in $n$ based on (4.9) and (4.10) yields

$$
r_{n}(\ell, \lambda)=\frac{\lambda^{2}}{4(n+1)(2 n+2 \ell+7)}+\frac{(4 n+2 \ell+5) \lambda}{2(n+1)(2 n+2 \ell+7)}+O_{n, \ell}(1) \quad \text { as } \quad \lambda \rightarrow \infty .
$$

This justifies the first two terms in (4.11). On the other side, we similarly obtain the following asymptotic

$$
r_{n}(\ell, \lambda)=\frac{3 \ell}{8(n+1)}+O_{n, \lambda}(1) \quad \text { as } \quad \ell \rightarrow+\infty .
$$

This gives rise to the last term in (4.11). Finally, to find an approximation to $r_{n}(\ell, \lambda)$ for small values of both $\ell$ and $\lambda$, we generate the sequence $\left\{r_{n}(0,0)\right\}_{1 \leq n \leq 20}{ }^{3}$ and fit to it an appropriate rational function in $n$. This leads to the choice of the remaining term in the approximation (4.11). We note that the procedure we used here to construct $\tilde{r}_{n}$ differs from the one in [12]. The new method is more transparent and much more efficient; in particular, it avoids polynomial approximation altogether in order to find multipliers of the powers of $\lambda$ in (4.11).

Now, to prove that $r_{n}$ and $\tilde{r}_{n}$ are indeed "close", we define

$$
\delta_{n}(\ell, \lambda):=\frac{r_{n}(\ell, \lambda)}{\tilde{r}_{n}(\ell, \lambda)}-1
$$

and show that $\delta_{n}$ is small uniformly in $\ell$ and $\lambda$. To that end we substitute Eq. (4.12) into Eq. (4.9) and derive the recurrence relation for $\delta_{n}$

$$
\delta_{n+1}=\varepsilon_{n}-C_{n} \frac{\delta_{n}}{1+\delta_{n}},
$$

where

$$
\varepsilon_{n}=\frac{A_{n} \tilde{r}_{n}+B_{n}}{\tilde{r}_{n} \tilde{r}_{n+1}}-1 \text { and } C_{n}=\frac{B_{n}}{\tilde{r}_{n} \tilde{r}_{n+1}} .
$$

Now, for every $\ell \geq 2, \lambda \in \overline{\mathbb{H}}$ and $n \geq 3$, we have the following estimates

$$
\begin{aligned}
\left|\delta_{3}(\ell, \lambda)\right| & \leq \frac{1}{3} \\
\left|\varepsilon_{n}(\ell, \lambda)\right| & \leq \frac{1}{12}+\frac{\ell}{6(\ell+n+5)}, \\
\left|C_{n}(\ell, \lambda)\right| & \leq \frac{1}{2}-\frac{\ell}{3(\ell+n+5)} .
\end{aligned}
$$

We illustrate the proof of the third estimate above; the other two are established analogously. Namely, we first bring $C_{n}(\ell, \lambda)$ to the form of the ratio of two polynomials $P_{1}(n, \ell, \lambda)$ and $P_{2}(n, \ell, \lambda)$. Also, to remove any possible ambiguity, we provide explicit forms of these polynomials in the Appendix B.1. Our aim is to establish the desired estimate on the imaginary line only, prove that $C_{n}(\ell, \lambda)$ is analytic and polynomially bounded for $\lambda \in \overline{\mathbb{H}}$ and then use Phragmén-Lindelöf principle to extend the estimate to the whole $\overline{\mathbb{H}}$. We therefore proceed by computing the polynomials

$$
Q_{1}(n, \ell, t):=\left|P_{1}(n, \ell, i t)\right|^{2} \quad \text { and } \quad Q_{2}(n, \ell, t):=\left|P_{2}(n, \ell, i t)\right|^{2},
$$

\footnotetext{
${ }^{3}$ Of course, the upper limit of this range can be any number that is large enough to serve the purpose. According to our experience, 20 is just fine.
} 
for $t$ real. Now, a straightforward calculation shows that the polynomial

$$
[6(\ell+n+10)]^{2} Q_{1}(n+3, \ell+2, t)-[\ell+3 n+26]^{2} Q_{2}(n+3, \ell+2, t)
$$

has manifestly negative coefficients (note the shift in $n$ and $\ell$ ). This in turn proves that for $\ell \geq 2$ and $n \geq 3$ the estimate for $C_{n}(\ell, \lambda)$ holds on the imaginary line. Since $C_{n}(\ell, \lambda)=$ $P_{1}(n, \ell, \lambda) / P_{2}(n, \ell, \lambda)$ is obviously polynomially bounded for $\lambda \in \overline{\mathbb{H}}$, it remains to prove that it is analytic there. This follows from the fact that for fixed $n \geq 3$ and $\ell \geq 2$, all of the zeros of $P_{2}(n, \ell, \cdot)$ are contained in the (open) left complex half plane. This can be shown in various ways. As a canonical method one can use for example Wall's formulation of the Routh-Hurwitz criterion, see [39].

Now, having established estimates (4.15), we employ a simple inductive argument to conclude from (4.13) that

$$
\left|\delta_{n}(\ell, \lambda)\right| \leq \frac{1}{3}
$$

for all $n \geq 3, \lambda \in \overline{\mathbb{H}}$ and $\ell \geq 2$. Since $\lim _{n \rightarrow \infty} \tilde{r}_{n}(\ell, \lambda)=1$, Eqns. (4.16) and (4.12) rule out (4.8) and we therefore finally conclude that (4.7) holds. This proves the proposition for $\ell \geq 2$.

4.1.3. The case $\ell=0$. By direct inspection one can check that for $\ell=0,\{1,3\} \subset \Sigma_{0}$ with respective solutions given by

$$
f_{0}(\rho ; 1)=\frac{1-\rho^{2}}{\left(1+\rho^{2}\right)^{2}} \quad \text { and } \quad f_{0}(\rho ; 3)=\frac{1}{\left(1+\rho^{2}\right)^{2}} .
$$

These are subsequently transformed (up to a constant multiple) into solutions of Eq. (4.3),

$$
y_{1}(x)=1-x \quad \text { and } \quad y_{3}(x)=1 .
$$

The fact that both functions in Eq. (4.18) are polynomials will be crucial for the rest of the proof. We now go on and prove that $\Sigma_{0}$ consists of $\lambda=1$ and $\lambda=3$ only. Let $\lambda \in \overline{\mathbb{H}} \backslash\{1,3\}$. We first observe that that $a_{n}(0, \lambda)$ can not be equal to zero eventually in $n$. Indeed, similarly to the case $\ell \geq 2$, let us assume that $a_{n}(0, \lambda)=0$ for some $\lambda \in \mathbb{H}$ and all $n \geq n_{0} \in \mathbb{N}$. This together with $a_{-1}(0, \lambda)=0$ (by backward substitution in Eq. (4.5)) yields $a_{0}(0, \lambda)=0$, unless $\lambda \in\{1,3\}^{4}$. We therefore conclude, by the theorem of Poincaré, that either Eq. (4.7) or Eq. (4.8) holds. Note that

$$
a_{2}(0, \lambda)=\frac{1}{2016}(\lambda-1)(\lambda-3)\left(\lambda^{2}+32 \lambda+235\right)
$$

and

We therefore let

$$
a_{3}(0, \lambda)=\frac{1}{266112}(\lambda-1)(\lambda-3)\left(\lambda^{4}+58 \lambda^{3}+1052 \lambda^{2}+6350 \lambda+4971\right) .
$$

$$
r_{2}(0, \lambda):=\frac{\lambda^{4}+58 \lambda^{3}+1052 \lambda^{2}+6350 \lambda+4971}{132\left(\lambda^{2}+32 \lambda+235\right)}
$$

and define $r_{n}(0, \lambda)$ for $n \geq 3$ by Eq. (4.9). In this way we have $r_{n}(0, \lambda)$ defined for all $\lambda \in \overline{\mathbb{H}}$. As an approximate solution, we use

$$
\tilde{r}_{n}(0, \lambda)=\frac{\lambda^{2}}{4(n+1)(2 n+7)}+\frac{(4 n+3) \lambda}{2(n+1)(2 n+7)}+\frac{n-1}{n+1},
$$

\footnotetext{
${ }^{4}$ This is in fact a reflection of the fact that the eigenfunctions corresponding to $\lambda=1$ and $\lambda=3$ are polynomials, see Eq. (4.18).
} 
instead of Eq. (4.11) (note the difference in the second term) and define $\delta_{n}, \varepsilon_{n}$ and $C_{n}$ as in Eq. (4.12)-(4.14). Now, we have the following estimates

$$
\left|\delta_{5}(0, \lambda)\right| \leq \frac{1}{3}, \quad\left|\varepsilon_{n}(0, \lambda)\right| \leq \frac{1}{12}, \quad \text { and } \quad\left|C_{n}(0, \lambda)\right| \leq \frac{1}{2},
$$

for all $n \geq 5$ and $\lambda \in \overline{\mathbb{H}}$. The proof is analogous to the one in the case $\ell \geq 2$ and the explicit forms are given in the Appendix B.1. Finally, by induction we show that $\left|\delta_{n}(0, \lambda)\right| \leq \frac{1}{3}$ for all $n \geq 5$. This rules out Eq. (4.8) and therefore Eq. (4.7) holds throughout $\overline{\mathbb{H}}$.

4.2. Proof of Proposition 4.2. By direct inspection one can check that for $\ell=1,\{0,1\} \subset$ $\Sigma_{1}$ with respective solutions given by

$$
f_{1}(\rho ; 0)=\frac{3 \rho-\rho^{3}}{\left(1+\rho^{2}\right)^{2}} \quad \text { and } \quad f_{1}(\rho ; 1)=\frac{\rho}{\left(1+\rho^{2}\right)^{2}} .
$$

To prove that there are no unstable eigenvalues other than $\lambda=0$ and $\lambda=1$ we closely follow the method developed in [12]. Namely, we first "remove" $\lambda=1$ and $\lambda=0$ by formulating another equation and then prove that the new equation is free of unstable eigenvalues. To proceed we first make a change of variables, namely we let $\hat{f}(\rho):=\rho f(\rho)$ and thereby transform Eq. (4.2) into

$$
\left(1-\rho^{2}\right) \hat{f}^{\prime \prime}(\rho)+\left[\frac{4}{\rho}-2(\lambda+1) \rho\right] \hat{f}^{\prime}(\rho)-\lambda(\lambda+1) \hat{f}(\rho)-\frac{2\left(5 \rho^{4}-14 \rho^{2}+5\right)}{\rho^{2}\left(1+\rho^{2}\right)^{2}} \hat{f}(\rho)=0 .
$$

Now, since $\hat{f}_{1}(\rho)=\frac{\rho^{2}}{\left(1+\rho^{2}\right)^{2}}$ solves Eq. (4.19) for $\lambda=1$ and does not vanish inside $(0,1)$, we remove $\lambda=1$ by the corresponding supersymmetric reformulation of Eq. (4.19), see Sec. 3 in [12]. Namely, we obtain

$$
\left(1-\rho^{2}\right) \tilde{f}^{\prime \prime}(\rho)+\left[\frac{4}{\rho}-2(\lambda+1) \rho\right] \tilde{f}^{\prime}(\rho)-\lambda(\lambda+1) \tilde{f}(\rho)+\frac{2\left(\rho^{6}+\rho^{4}-\rho^{2}-9\right)}{\rho^{2}\left(1+\rho^{2}\right)^{2}} \tilde{f}(\rho)=0 .
$$

Direct computation shows that $\tilde{f}_{0}(\rho)=\frac{\rho^{3}}{1+\rho^{2}}$ solves Eq. (4.20) for $\lambda=0$. Furthermore, since $\tilde{f}_{0}$ has no zeros within $(0,1)$, by following the process in Sec. 3 in [12] (while making necessary alterations) we perform supersymmetric removal of $\lambda=0$ relative to Eq. (4.20). The equation we thereby get is

$$
\left(1-\rho^{2}\right) \tilde{f}^{\prime \prime}(\rho)+\left[\frac{4}{\rho}-2(\lambda+1) \rho\right] \tilde{f}^{\prime}(\rho)-\lambda(\lambda+1) \tilde{f}(\rho)-\frac{4\left(\rho^{2}+7\right)}{\rho^{2}\left(1+\rho^{2}\right)} \tilde{f}(\rho)=0 .
$$

It remains to prove that Eq. (4.21) has no smooth solutions for $\operatorname{Re} \lambda \geq 0$. To that end we proceed as in the proof of Proposition 4.1. Namely, we first define

$$
\rho=\sqrt{\frac{x}{2-x}} \text { and } \tilde{f}(\rho)=x^{2}(2-x)^{\frac{\lambda}{2}} y(x),
$$

and thereby transform Eq. (4.21) into a Heun equation

$$
y^{\prime \prime}(x)+\left(\frac{13}{2 x}+\frac{\lambda-1}{x-1}+\frac{1}{2(x-2)}\right) y^{\prime}(x)+\frac{(\lambda+6)(\lambda+4) x-\lambda^{2}-22 \lambda-48}{4 x(x-1)(x-2)} y(x)=0 .
$$


Then we consider the normalized analytic solution of Eq. (4.22) at $x=0$

$$
\sum_{n=0}^{\infty} a_{n}(\lambda) x^{n}, \quad a_{0}(\lambda)=1
$$

The recurrence relation that the sequence of coefficients $\left\{a_{n}(\lambda)\right\}_{n \geq 0}$ obeys is

$$
a_{n+2}(\lambda)=A_{n}(\lambda) a_{n+1}(\lambda)+B_{n}(\lambda) a_{n}(\lambda),
$$

where

$$
A_{n}(\lambda)=\frac{\lambda^{2}+2(4 n+15) \lambda+12(n+5)(n+2)}{4(2 n+15)(n+2)}
$$

and

$$
B_{n}(\lambda)=-\frac{(\lambda+2 n+6)(\lambda+2 n+4)}{4(2 n+15)(n+2)} .
$$

and $a_{-1}(\lambda)=0$ and $a_{0}(\lambda)=1$. We now let $r_{n}(\lambda):=\frac{a_{n+1}(\lambda)}{a_{n}(\lambda)}$, and thereby transform Eq. (4.23) into

$$
r_{n+1}(\lambda)=A_{n}(\lambda)+\frac{B_{n}(\lambda)}{r_{n}(\lambda)}
$$

with the initial condition

$$
r_{0}(\lambda)=\frac{a_{1}(\lambda)}{a_{0}(\lambda)}=A_{-1}(\lambda)=\frac{\lambda^{2}}{52}+\frac{11 \lambda}{26}+\frac{12}{13} .
$$

By backwards substitution we see that if $\lambda \in \overline{\mathbb{H}}$ then $a_{n}(\lambda)$ can not be eventually zero. Also, by Poincaré's theorem we have again either $\lim _{n \rightarrow \infty} r_{n}(\lambda)=1$ or $\lim _{n \rightarrow \infty} r_{n}(\lambda)=\frac{1}{2}$. Then, by observing the behavior of $r_{n}$ for different values of $\lambda$ we construct the following approximate solution to Eq. (4.24)

$$
\tilde{r}_{n}(\lambda):=\frac{\lambda^{2}}{4(2 n+13)(n+1)}+\frac{(2 n+5) \lambda}{(2 n+13)(n+1)}+\frac{2 n+9}{2 n+13} .
$$

Subsequently we define $\delta_{n}(\lambda):=\frac{r_{n}(\lambda)}{\tilde{r}_{n}(\lambda)}-1$, and derive the corresponding recurrence relation

$$
\delta_{n+1}=\varepsilon_{n}-C_{n} \frac{\delta_{n}}{1+\delta_{n}},
$$

where $\varepsilon_{n}=\frac{A_{n} \tilde{r}_{n}+B_{n}}{\tilde{r}_{n} \tilde{r}_{n+1}}-1$ and $C_{n}=\frac{B_{n}}{\tilde{r}_{n} \tilde{r}_{n+1}}$. Now for every $n \geq 1$ and $\lambda \in \overline{\mathbb{H}}$ the following estimates hold

$$
\left|\delta_{1}(\lambda)\right| \leq \frac{1}{3}, \quad\left|\varepsilon_{n}(\lambda)\right| \leq \frac{1}{12}, \quad\left|C_{n}(\lambda)\right| \leq \frac{1}{2} .
$$

They are proven in the same way as the corresponding ones in Proposition 4.1; the relevant explicit expressions are given in Appendix B.2. Finally, by induction we conclude that $\delta_{n}(\lambda) \leq \frac{1}{3}$ for all $n \geq 1$ and $\lambda \in \overline{\mathbb{H}}$. This and the fact that $\lim _{n \rightarrow \infty} \tilde{r}_{n}(\lambda)=1$ rule out $\lim _{n \rightarrow \infty} r_{n}(\lambda)=\frac{1}{2}$. Therefore $\lim _{n \rightarrow \infty} r_{n}(\lambda)=1$ holds throughout $\overline{\mathbb{H}}$. This finishes the proof.

Remark 4.3. In the case $\ell=0$, both solutions in (4.17) are nonzero on the interval $(0,1)$. However, when trying to perform a supersymmetric reformulations of Eq. (4.2) as in the case $\ell=1$, the following scenario occurs: the supersymmetric reformulation relative to either of the two values $\lambda=1$ and $\lambda=3$ is the same and it removes $\lambda=3$ only. What is more, any further attempt to remove $\lambda=1$ in this way, fails. Therefore, a different approach was 
required. The new method we devised in Section 4.1.3 crucially relies on the fact that the function in Eq. (4.18) are polynomials. The process does not require any reformulation of Eq. (4.3) and even allows for solutions that vanish inside $(0,1)$. For that reason, the method is, in a sense, more general than the one exhibited in [12] and would in addition yield even shorter solutions to similar spectral problems in $[13,12,16]$.

\section{The spectrum of $\mathbf{L}_{a}-$ Growth Bounds for $\mathbf{S}_{a}(\tau)$}

With the result of the previous section, we are now able to investigate the spectrum of the operator $\mathbf{L}_{a}: \mathcal{D}\left(\mathbf{L}_{a}\right) \subset \mathcal{H} \rightarrow \mathcal{H}$. First, we make some general observations.

Lemma 5.1. Fix $\varepsilon>0$. There are constants $\kappa^{*}>0$ and $c>0$ such that for all $a \in \overline{\mathbb{B}_{\delta}^{7}}$ with $\delta>0$ sufficiently small

$$
\left\|\mathbf{R}_{\mathbf{L}_{a}}(\lambda)\right\| \leq c
$$

for all $\lambda \in \mathbb{C}$ satisfying $\operatorname{Re} \lambda \geq-\frac{1}{2}+\varepsilon$ and $|\lambda| \geq \kappa^{*}$.

Proof. In view of Proposition 3.4 and the identity

$$
\lambda-\mathbf{L}_{a}=\left[1-\mathbf{L}_{a}^{\prime} \mathbf{R}_{\mathbf{L}}(\lambda)\right](\lambda-\mathbf{L})
$$

it suffices to show that under suitable assumptions on $a$ and $\lambda,\left\|\mathbf{L}_{a}^{\prime} \mathbf{R}_{\mathbf{L}}(\lambda)\right\| \leq \frac{1}{2}$ such that the Neumann series $\sum_{k=0}^{\infty}\left[\mathbf{L}_{a}^{\prime} \mathbf{R}_{\mathbf{L}}(\lambda)\right]^{k}$ converges. Then $\mathbf{R}_{\mathbf{L}_{a}}(\lambda)=\mathbf{R}_{\mathbf{L}}(\lambda) \sum_{k=0}^{\infty}\left[\mathbf{L}_{a}^{\prime} \mathbf{R}_{\mathbf{L}}(\lambda)\right]^{k}$ and the claimed bounds follow from Proposition 3.4. By definition of $\mathbf{L}_{a}^{\prime}$ and the properties of the potential we have

$$
\left\|\mathbf{L}_{a}^{\prime} \mathbf{R}_{\mathbf{L}}(\lambda) \mathbf{f}\right\|=\left\|V_{a}\left[\mathbf{R}_{\mathbf{L}}(\lambda) \mathbf{f}\right]_{1}\right\|_{H^{2}\left(\mathbb{B}^{7}\right)} \lesssim\left\|\left[\mathbf{R}_{\mathbf{L}}(\lambda) \mathbf{f}\right]_{1}\right\|_{H^{2}\left(\mathbb{B}^{7}\right)},
$$

for all $a \in \overline{\mathbb{B}_{\delta}^{7}}$ with $0<\delta<\delta^{*}$ suitably small. To estimate the last term, we use the structure of $\mathbf{L}$. For $\mathbf{f} \in \mathcal{H}$, set $\mathbf{u}=\mathbf{R}_{\mathbf{L}}(\lambda) \mathbf{f} \in \mathcal{D}(\mathbf{L})$ such that $(\lambda-\mathbf{L}) \mathbf{u}=\mathbf{f}$. The first component of this equation reads

Hence,

$$
\xi^{i} \partial_{i} u_{1}(\xi)+(\lambda+1) u_{1}(\xi)-u_{2}(\xi)=f_{1}(\xi)
$$

$$
\left\|u_{1}\right\|_{H^{2}\left(\mathbb{B}^{7}\right)} \lesssim \frac{1}{|\lambda+1|}\left(\left\|u_{1}\right\|_{H^{3}\left(\mathbb{B}^{7}\right)}+\left\|u_{2}\right\|_{H^{2}\left(\mathbb{B}^{7}\right)}+\left\|f_{1}\right\|_{H^{2}\left(\mathbb{B}^{7}\right)}\right) .
$$

With Proposition 3.4 we infer that

$$
\left\|\left[\mathbf{R}_{\mathbf{L}}(\lambda) \mathbf{f}\right]_{1}\right\|_{H^{2}\left(\mathbb{B}^{7}\right)} \lesssim|\lambda|^{-1}\left\|\left(\left\|\mathbf{R}_{\mathbf{L}}(\lambda) \mathbf{f}\right\|+\|\mathbf{f}\|\right) \lesssim|\lambda|^{-1}\right\| \mathbf{f} \|
$$

for all $\lambda \in \mathbb{C}$ with $\operatorname{Re} \lambda \geq-\frac{1}{2}+\varepsilon$ such that

$$
\left\|\mathbf{L}_{a}^{\prime} \mathbf{R}_{\mathbf{L}}(\lambda) \mathbf{f}\right\| \lesssim|\lambda|^{-1}\|\mathbf{f}\|
$$

for $a$ sufficiently small. The statement holds if $|\lambda| \geq \kappa^{*}$ for some suitably large $\kappa^{*}>0$.

Lemma 5.2. Let $\delta>0$ be sufficiently small and $a \in \overline{\mathbb{B}_{\delta}^{7}}$. If $\lambda \in \sigma\left(\mathbf{L}_{a}\right)$ and $\operatorname{Re} \lambda>-\frac{1}{2}$, then $\lambda$ is an isolated eigenvalue.

Proof. The assumptions on $\lambda$ imply that $\lambda \notin \sigma(\mathbf{L})$. We use again Eq. (5.1) to see that $\lambda \in \sigma\left(\mathbf{L}_{a}\right)$ if and only if the operator $1-\mathbf{L}_{a}^{\prime} \mathbf{R}_{\mathbf{L}}(\lambda)$ is not bounded invertible. This means that 1 is an eigenvalue of the compact operator $\mathbf{L}_{a}^{\prime} \mathbf{R}_{\mathbf{L}}(\lambda)$. Hence, there is an eigenfunction $\mathbf{f} \in \mathcal{H}$ satisfying $\left(1-\mathbf{L}_{a}^{\prime} \mathbf{R}_{\mathbf{L}}(\lambda)\right) \mathbf{f}=0$. We set $\mathbf{u}:=\mathbf{R}_{\mathbf{L}}(\lambda) \mathbf{f} \in \mathcal{D}(\mathbf{L})=\mathcal{D}\left(\mathbf{L}_{a}\right)$. Then $(\lambda-\mathbf{L}) \mathbf{u}=\mathbf{f}$ and infer that $\left(\lambda-\mathbf{L}_{a}\right) \mathbf{u}=\left(1-\mathbf{L}_{a}^{\prime} \mathbf{R}_{\mathbf{L}}(\lambda)\right)(\lambda-\mathbf{L}) \mathbf{u}=0$. We conclude that $\lambda$ is an eigenvalue 
of $\mathbf{L}_{a}$. Next, we apply the Analytic Fredholm Theorem, see e.g. [38], Theorem 3.14.3, p. 194, to the function $\lambda \mapsto \mathbf{L}_{a}^{\prime} \mathbf{R}_{\mathbf{L}}(\lambda)$ defined on the open half plane $\mathbb{H}_{-\frac{1}{2}}:=\left\{\lambda \in \mathbb{C}: \operatorname{Re} \lambda>-\frac{1}{2}\right\}$. By Lemma 5.1, there are points in $\mathbb{H}_{-\frac{1}{2}}$ such that $1-\mathbf{L}_{a}^{\prime} \mathbf{R}_{\mathbf{L}}(\lambda)$ is invertible. Thus, it is invertible on all of $\mathbb{H}_{-\frac{1}{2}}$ except for a discrete set $S \subset \mathbb{H}_{-\frac{1}{2}}$ and we infer that $\lambda$ is isolated.

\subsection{The spectrum of $\mathbf{L}_{a}, a=0$.}

Proposition 5.3. There exists an $0<\omega_{0} \leq \frac{1}{2}$, such that

$$
\sigma\left(\mathbf{L}_{0}\right) \subset\left\{\lambda \in \mathbb{C}: \operatorname{Re} \lambda \leq-\omega_{0}\right\} \cup\left\{\lambda_{0}, \lambda_{1}, \lambda_{2}\right\},
$$

where $\lambda_{0}=0, \lambda_{1}=1$ and $\lambda_{2}=3$ are eigenvalues. The geometric eigenspace of the eigenvalue $\lambda_{2}$ is one-dimensional and spanned by $\mathbf{h}_{0}=\left(h_{0,1}, h_{0,2}\right)$,

$$
h_{0,1}(\xi)=\frac{1}{\left(1+|\xi|^{2}\right)^{2}}, \quad h_{0,2}(\xi)=4 h_{0,1}(\xi)+\xi^{j} \partial_{j} h_{0,1}(\xi) .
$$

Furthermore, the geometric eigenspaces of $\lambda_{1}$ and $\lambda_{0}$ are spanned by functions $\left\{\mathbf{g}_{0}^{(k)}\right\}_{k=0, \ldots, 7}$, and $\left\{\mathbf{q}_{0}^{(j)}\right\}_{j=1, \ldots, 7}$, respectively. Explicitly, we have

$$
\begin{aligned}
& g_{0,1}^{(0)}(\xi)=\frac{\left(1-|\xi|^{2}\right)}{\left(1+|\xi|^{2}\right)^{2}}, \quad g_{0,2}^{(0)}(\xi)=2 g_{0,1}^{(0)}(\xi)+\xi^{j} \partial_{j} g_{0,1}^{(0)}(\xi), \\
& g_{0,1}^{(j)}(\xi)=\frac{\xi_{j}}{\left(1+|\xi|^{2}\right)^{2}}, \quad g_{0,2}^{(j)}(\xi)=2 g_{0,1}^{(j)}(\xi)+\xi^{j} \partial_{j} g_{0,1}^{(j)}(\xi),
\end{aligned}
$$

and

$$
q_{0,1}^{(j)}(\xi)=\frac{\xi_{j}\left(3-|\xi|^{2}\right)}{\left(1+|\xi|^{2}\right)^{2}}, \quad q_{0,2}^{(j)}(\xi)=q_{0,1}^{(j)}(\xi)+\xi^{j} \partial_{j} q_{0,1}^{(j)}(\xi)
$$

Proof. We prove that

$$
\{\lambda \in \mathbb{C}: \operatorname{Re} \lambda \geq 0\} \backslash\{0,1,3\} \subset \rho\left(\mathbf{L}_{0}\right) .
$$

Let $\operatorname{Re} \lambda \geq 0$ and assume that $\lambda \in \sigma\left(\mathbf{L}_{0}\right)$. By Lemma 5.2, $\lambda$ is an eigenvalue and there is a non-trivial $\mathbf{u} \in \mathcal{D}\left(\mathbf{L}_{0}\right)$ satisfying the eigenvalue equation $\left(\lambda-\mathbf{L}_{0}\right) \mathbf{u}=0$ (in a suitable weak sense). As in the proof of Lemma 3.3 this equation reduces to a degenerate elliptic problem for $u_{1}$

$$
-\left(\delta^{i j}-\xi^{i} \xi^{j}\right) \partial_{i} \partial_{j} u_{1}(\xi)+2(\lambda+2) \xi^{i} \partial_{i} u_{1}(\xi)+(\lambda+1)(\lambda+2) u_{1}(\xi)-V_{0}(\xi) u_{1}(\xi)=0
$$

and $u_{2}$ is given by

$$
u_{2}(\xi)=\xi^{i} \partial_{i} u_{1}(\xi)+(\lambda+1) u_{1}(\xi) .
$$

Now, $\mathbf{u} \in \mathcal{H}$ implies that $u_{1} \in H^{3}\left(\mathbb{B}^{7}\right)$ and by elliptic regularity, we infer that $u_{1} \in C^{\infty}\left(\mathbb{B}^{7}\right) \cap$ $H^{3}\left(\mathbb{B}^{7}\right)$. Since the potential $V_{0}$ is radial we can do a decomposition into spherical harmonics. We expand $u_{1}$ according to

$$
u_{1}(\rho \omega)=\sum_{\ell=0}^{\infty} \sum_{m \in \Omega_{\ell}}\left(u_{1}(\rho \cdot) \mid Y_{\ell, m}\right)_{L^{2}\left(\mathbb{S}^{d-1}\right)} Y_{\ell, m}(\omega)=: \sum_{\ell=0}^{\infty} \sum_{m \in \Omega_{\ell}} u_{\ell, m}(\rho) Y_{\ell, m}(\omega),
$$


where the sum converges in $H^{k}\left(\mathbb{B}_{1-\varepsilon}^{7}\right)$ for any $k \in \mathbb{N}_{0}$ and fixed $\varepsilon>0$, see Eq. (1.13). With this Eq. (5.5) decouples into infinitely many ODEs. The fact that $u_{1}$ is non-trivial implies that there are indices $\ell \in \mathbb{N}_{0}, m \in \Omega_{\ell}$, such that that $u_{\ell, m}$ is non-trivial and satisfies

$$
\mathcal{T}_{\ell}(\lambda) u_{\ell, m}(\rho)=0,
$$

where $\mathcal{T}_{\ell}(\lambda)$ is given in Eq. (4.1). The properties of $u_{1}$ imply that $u_{\ell, m} \in C^{\infty}[0,1) \cap H^{3}\left(\frac{1}{2}, 1\right)$. By inspection, the Frobenius indices at $\rho=1$ are $\{0,2-\lambda\}$. If $\lambda \notin \mathbb{N}_{0}$, then $u_{\ell, m}$ is either analytic or it behaves like $(1-\rho)^{2-\lambda}$. For $\lambda \in \mathbb{N}, \lambda \geq 3$, we come to the same conclusion. If $\lambda=2,1$ or 0 then the non-analytic behavior is described by $\log (1-\rho), \log (1-\rho)(1-\rho)$ and $\log (1-\rho)(1-\rho)^{2}$, respectively. In all cases, non-analyticity can be excluded by the requirement $u_{\ell, m} \in H^{3}\left(\frac{1}{2}, 1\right)$ and we infer that $u_{\ell, m} \in C^{\infty}[0,1]$. In view of the Propositions 4.1 - 4.2 we have $\ell \in\{0,1\}$ and we conclude that if $\lambda \notin\{0,1,3\}$ then $\lambda \in \rho\left(\mathbf{L}_{0}\right)$ by contradiction.

If $\lambda=3$, then $\ell=0$ and $u_{0, m}(\rho)=\left(1+\rho^{2}\right)^{-2}$ where $m \in \Omega_{m}=\{1\}$, which implies Eq. (5.2) since $Y_{0, m}$ is a constant. If $\lambda=1$, then either $\ell=0$ and $u_{0, m}=f_{0}(\cdot ; 1)$ or $\ell=1$ and $u_{1, m}=f_{1}(\cdot ; 1)$. Since $Y_{1, m}(\omega)$ is a constant multiple of $\omega^{m}$ for $m=1, \ldots, 7$, we obtain Eq. (5.3). Finally, if $\lambda=0$, then $\ell=1$ and $u_{1, m}=f_{1}(\cdot ; 0)$, which yields Eq. (5.4). The claim now follows from the openness of $\rho(\mathbf{L})$, Lemma 5.1 and Lemma 5.2.

5.1.1. Spectral projections. We define Riesz projections associated to the unstable eigenvalues of $\mathbf{L}_{0}$. We set

$$
\mathbf{H}_{0}:=\frac{1}{2 \pi i} \int_{\gamma_{2}} \mathbf{R}_{\mathbf{L}_{0}}(\lambda) d \lambda \quad \mathbf{P}_{0}:=\frac{1}{2 \pi i} \int_{\gamma_{1}} \mathbf{R}_{\mathbf{L}_{0}}(\lambda) d \lambda, \quad \mathbf{Q}_{0}:=\frac{1}{2 \pi i} \int_{\gamma_{0}} \mathbf{R}_{\mathbf{L}_{0}}(\lambda) d \lambda
$$

where $\gamma_{j}(s):=\lambda_{j}+\frac{\omega_{0}}{2} e^{2 \pi i s}$ for $s \in[0,1]$.

Lemma 5.4. We have

$$
\operatorname{dim} \operatorname{rg} \mathbf{H}_{0}=1, \quad \operatorname{dim} \operatorname{rg} \mathbf{P}_{0}=8, \quad \operatorname{dim} \operatorname{rg} \mathbf{Q}_{0}=7 .
$$

Proof. First, we observe that the projection operators have finite rank. If this was not the case, they would belong to the the essential spectrum of $\mathbf{L}_{a}$ see [26], p. 239, Theorem 5.28 and (5.33) on p. 242. However, since the essential spectrum is invariant under compact perturbations, see [26], Theorem 5.35, p. 244, this contradicts Proposition 3.4. Next, we show that $\operatorname{dim} \operatorname{rg} \mathbf{P}_{0}=8$ (the abstract part of the argument follows analogously for the other projections). First, we have $\mathcal{H}=\operatorname{ker} \mathbf{P}_{0} \oplus \operatorname{rg} \mathbf{P}_{0}$ and $\mathbf{L}_{0}$ is decomposed into parts on the respective subspaces. Note that $\operatorname{rg} \mathbf{P}_{0} \subset \mathcal{D}\left(\mathbf{L}_{0}\right)$ and that the spectrum of the part of $\mathbf{L}_{0}$ on $\operatorname{rg} \mathbf{P}_{0}$ is given by

$$
\sigma\left(\left.\mathbf{L}_{0}\right|_{\operatorname{rg} \mathbf{P}_{0}}\right)=\{1\} .
$$

Since $\mathbf{P}_{0}$ has finite rank, $\left.\mathbf{L}_{0}\right|_{\mathrm{rg}} \mathbf{P}_{0}$ is a bounded operator. We now consider the operator $\lambda-\left.\mathbf{L}_{0}\right|_{\mathrm{rg} \mathbf{P}_{0}}$ for $\lambda=1$, which is nilpotent due to the spectral structure of $\left.\mathbf{L}_{0}\right|_{\mathrm{rg} \mathbf{P}_{0}}$. Hence, there exists a minimal $m \in \mathbb{N}$ such that

$$
\left(\lambda-\left.\mathbf{L}_{0}\right|_{\operatorname{rg} \mathbf{P}_{0}}\right)^{m} \mathbf{u}=0
$$

for $\lambda=1$ and all $\mathbf{u} \in \operatorname{rg} \mathbf{P}_{0}$. Obviously, $\operatorname{ker}\left(1-\mathbf{L}_{0}\right)=\operatorname{span}\left(\mathbf{g}_{0}^{(0)}, \mathbf{g}_{0}^{(1)}, \ldots, \mathbf{g}_{0}^{(7)}\right) \subset \operatorname{rg} \mathbf{P}_{0}$. If $m=1$, then the reverse inclusion holds and the claim follows. We argue by contradiction and assume that $m \geq 2$. Then there is $\mathbf{u} \in \operatorname{rg} \mathbf{P}_{0}$ such that

$$
\left(1-\mathbf{L}_{0}\right) \mathbf{u}=\mathbf{v},
$$


for some nontrivial $\mathbf{v} \in \operatorname{ker}\left(1-\mathbf{L}_{0}\right)$. Eq. (5.8) yields an elliptic equation for $u_{1}$ given by

$$
-\left(\delta^{i j}-\xi^{i} \xi^{j}\right) \partial_{i} \partial_{j} u_{1}(\xi)+2(\lambda+2) \xi^{i} \partial_{i} u_{1}(\xi)+(\lambda+1)(\lambda+2) u_{1}(\xi)-V_{0}(\xi) u_{1}(\xi)=F(\xi),
$$

for $\lambda=1$, where

$$
F(\xi)=\xi^{i} \partial_{i} v_{1}(\xi)+(\lambda+2) v_{1}(\xi)+v_{2}(\xi) .
$$

Now, $\mathbf{v} \in \operatorname{ker}\left(1-\mathbf{L}_{0}\right)$ implies that $\mathbf{v}=\sum_{k=0}^{7} \tilde{\alpha_{k}} \mathbf{g}_{0}^{(k)}$ for some constants $\tilde{\alpha_{k}} \in \mathbb{C}$, see Proposition 5.3. To abbreviate the notation we set $g_{k}:=g_{0,1}^{(k)}$ to obtain

$$
F(\xi)=\sum_{k=0}^{7} \tilde{\alpha_{k}}\left(2 \xi^{i} \partial_{i} g_{k}+5 g_{k}\right)
$$

Note that $g_{0}(\xi)$ and $g_{j}(\xi)$ are constant multiples of $f_{0}(|\xi|) Y_{0,1}\left(\frac{\xi}{|\xi|}\right)$ and $f_{1}(|\xi|) Y_{1, j}\left(\frac{\xi}{|\xi|}\right)$ respectively, for $j=1, \ldots, 7$, with $f_{0}=f_{0}(\cdot ; 1), f_{1}=f_{1}(\cdot ; 1)$ defined in Proposition 4.1 and Proposition 4.2. Hence, in polar coordinates the right hand side of Eq. (5.9) can be written as

$$
F(\rho \omega)=\alpha_{0}\left[2 \rho f_{0}^{\prime}(\rho)+5 f_{0}(\rho)\right] Y_{0,1}(\omega)+\sum_{j=1}^{7} \alpha_{j}\left[2 \rho f_{1}^{\prime}(\rho)+5 f_{1}(\rho)\right] Y_{1, j}(\omega)
$$

with $\alpha_{k} \neq 0$ for at least one $k \in\{0, \ldots, 7\}$. Since $u_{1}$ is smooth on $\mathbb{B}^{7}$ we decompose it into spherical harmonics and set $u_{\ell, m}(\rho):=\left(u_{1}(\rho \cdot) \mid Y_{\ell, m}\right)$. The properties of $u_{1}$ imply that $u_{\ell, m} \in C^{\infty}[0,1) \cap H^{3}\left(\frac{1}{2}, 1\right)$ such that $u_{\ell, m} \in C^{2}[0,1]$ by Sobolev embedding. In view of Eq. (5.9) the following ODEs are satisfied,

$$
\mathcal{T}_{0}(1) u_{0,1}=-\alpha_{0} G_{0}, \quad \mathcal{T}_{1}(1) u_{1, j}=-\alpha_{j} G_{1},
$$

for $j=1, \ldots, 7$ and $G_{i}(\rho)=2 \rho f_{i}^{\prime}(\rho)+5 f_{i}(\rho), i=0,1$. Assume that $\alpha_{0} \neq 0$. Without loss of generality we set $\alpha_{0}=-1$ and study the ODE problem

$$
\left(1-\rho^{2}\right) u^{\prime \prime}(\rho)+\left(\frac{6}{\rho}-6 \rho\right) u^{\prime}(\rho)-\left(6-\frac{48}{\left(1+\rho^{2}\right)^{2}}\right) u(\rho)=G_{0}(\rho)
$$

with

$$
G_{0}(\rho)=-\frac{\rho^{4}+12 \rho^{2}-5}{\left(1+\rho^{2}\right)^{3}}
$$

We claim that there is no $C^{2}[0,1]$ solution to Eq. (5.11). For the homogeneous version of that equation a fundamental system is given by $\left\{\hat{u}_{1}, \hat{u}_{2}\right\}$, where

$$
\hat{u}_{1}(\rho)=f_{0}(\rho)=\frac{1-\rho^{2}}{\left(1+\rho^{2}\right)^{2}}
$$

and

$$
\hat{u}_{2}(\rho)=\hat{u}_{1}(\rho) \int_{\frac{1}{2}}^{\rho} \frac{d s}{s^{6} \hat{u}_{1}(s)^{2}}=\frac{1-\rho^{2}}{\left(1+\rho^{2}\right)^{2}} \int_{\frac{1}{2}}^{\rho} \frac{\left(1+s^{2}\right)^{4}}{s^{6}\left(1-s^{2}\right)^{2}} d s
$$

for $\rho \in(0,1)$. Note that

$$
\hat{u}_{2}(\rho) \simeq \rho^{-5} \quad \text { as } \quad \rho \rightarrow 0^{+}
$$

and

$$
\hat{u}_{2}(\rho)=2-6(1-\rho) \ln (1-\rho)+O(1-\rho) \quad \text { as } \quad \rho \rightarrow 1^{-} \text {. }
$$


Since $W\left(\hat{u}_{1}, \hat{u}_{2}\right)(\rho)=\rho^{-6}$, we solve Eq. (5.11) by the method of variation of parameters. Namely, for $\rho \in(0,1)$ we have

$$
u(\rho)=c_{1} \hat{u}_{1}(\rho)+c_{2} \hat{u}_{2}(\rho)+\hat{u}_{2}(\rho) \int_{0}^{\rho} \frac{\hat{u}_{1}(s) G_{0}(s) s^{6}}{1-s^{2}} d x-\hat{u}_{1}(\rho) \int_{0}^{\rho} \frac{\hat{u}_{2}(s) G_{0}(s) s^{6}}{1-s^{2}} d s
$$

for some $c_{1}, c_{2} \in \mathbb{C}$. If $u \in C^{2}[0,1]$ then $c_{2}=0$ in the above expression. Subsequently, by differentiating Eq. (5.14) we get

$$
u^{\prime}(\rho)=c_{1} \hat{u}_{1}^{\prime}(\rho)+\hat{u}_{2}^{\prime}(\rho) \int_{0}^{\rho} \frac{\hat{u}_{1}(s) G_{0}(s) s^{6}}{1-s^{2}} d x-\hat{u}_{1}^{\prime}(\rho) \int_{0}^{\rho} \frac{\hat{u}_{2}(s) G_{0}(s) s^{6}}{1-s^{2}} d s,
$$

for $\rho \in(0,1)$. We claim that

$$
u^{\prime}(\rho) \simeq \ln (1-\rho) \quad \text { as } \quad \rho \rightarrow 1^{-} .
$$

To establish this we study the asymptotics of all three terms on the right hand side of Eq. (5.15). First of all, the first term is bounded near $\rho=1$. Then, from

$$
C:=\int_{0}^{1} \frac{\hat{u}_{1}(s) G_{0}(s) s^{6}}{1-s^{2}} d s=\int_{0}^{1} \frac{s^{2}}{1-s^{2}} \frac{d}{d s}\left[\frac{s^{5}\left(1-s^{2}\right)^{2}}{\left(1+s^{2}\right)^{4}}\right] d s=-2 \int_{0}^{1} \frac{s^{6}}{\left(1+s^{2}\right)^{4}} d s<0
$$

and Eq. (5.13) we have

$$
\hat{u}_{2}^{\prime}(\rho) \int_{0}^{\rho} \frac{\hat{u}_{1}(s) G_{0}(s) s^{6}}{1-s^{2}} d s \sim 6 C \ln (1-\rho) \quad \text { as } \quad \rho \rightarrow 1^{-} .
$$

Also,

$$
\hat{u}_{1}^{\prime}(\rho) \int_{0}^{\rho} \frac{\hat{u}_{2}(s) G(s) s^{6}}{1-s^{2}} d s \sim-\frac{1}{2} \ln (1-\rho) \quad \text { as } \quad \rho \rightarrow 1^{-} .
$$

Now we easily observe that $6 C+\frac{1}{2}>0$ and then infer from these asymptotics and Eq. (5.15) that (5.16) holds. We therefore conclude that there is no $C^{2}[0,1]$ solution to Eq. (5.11) and hence $\alpha_{0}=0$.

Then, $\alpha_{j} \neq 0$ for at least one $j \in\{1, \ldots, 7\}$. Without loss of generality, we set $\alpha_{1}=-1$. Eq. (5.10) implies that the ODE

$$
\left(1-\rho^{2}\right) u^{\prime \prime}(\rho)+\left(\frac{6}{\rho}-6 \rho\right) u^{\prime}(\rho)-\left(6+\frac{6}{\rho^{2}}-\frac{48}{\left(1+\rho^{2}\right)^{2}}\right) u(\rho)=G_{1}(\rho)
$$

with $G_{1}(\rho)=\frac{\rho\left(7-\rho^{2}\right)}{\left(1+\rho^{2}\right)^{2}}$ has a solution which is in $C^{2}[0,1]$. We will again show that this is impossible. More precisely, we prove that any solution to Eq. (5.17) that is bounded near $\rho=0$ has an unbounded derivative near $\rho=1$. Note that $\hat{u}_{1}(\rho)=f_{1}(\rho)=\frac{\rho}{\left(1+\rho^{2}\right)^{2}}$, solves the homogeneous version of Eq. (5.17). We can therefore compute another solution, namely

$$
\hat{u}_{2}(\rho)=\hat{u}_{1}(\rho) \int_{1}^{\rho} \frac{d s}{s^{6} \hat{u}_{1}(s)^{2}},
$$

such that $\hat{u}_{1}$ and $\hat{u}_{2}$ are linearly independent. In particular, we have $\hat{u}_{2}(\rho) \simeq \rho^{-6}$ as $\rho \rightarrow 0^{+}$ and $\hat{u}_{2}(\rho) \simeq 1-\rho$ as $\rho \rightarrow 1^{-}$. Now, the general solution to Eq. (5.17) for $\rho \in(0,1)$ is

$$
u(\rho)=c_{1} \hat{u}_{1}(\rho)+c_{2} \hat{u}_{2}(\rho)+\hat{u}_{2}(\rho) \int_{0}^{\rho} \frac{\hat{u}_{1}(s) G_{1}(s) s^{6}}{1-s^{2}} d s-\hat{u}_{1}(\rho) \int_{0}^{\rho} \frac{u_{2}(s) G_{1}(s) s^{6}}{1-s^{2}} d s .
$$

Boundedness of $u$ at the origin implies $c_{2}=0$. Then we simply observe that $u^{\prime}(\rho) \simeq \ln (1-\rho)$ as $\rho \rightarrow 1^{-}$. We finally infer that $m=1$ in Eq. (5.7), which in turn implies the claim for $\mathbf{P}_{0}$. 
For $\mathbf{H}_{0}$, the right hand side of the analogue of Eq. (5.9) is given by $F(\xi)=2 \xi^{j} \partial_{j} h_{0,1}(\xi)+$ $9 h_{0,1}(\xi)$ which leads to the claim that the ODE

$$
\left(1-\rho^{2}\right) u^{\prime \prime}(\rho)+\left(\frac{6}{\rho}-10 \rho\right) u^{\prime}(\rho)-\left(20-\frac{48}{\left(1+\rho^{2}\right)^{2}}\right) u(\rho)=H(\rho)
$$

with $H(\rho)=\frac{9+\rho^{2}}{\left(1+\rho^{2}\right)^{3}}$ has a solution $u \in C^{2}[0,1]$. However, we exclude this in a similar way as above. Namely, we show that every solution to Eq. (5.18) that is bounded near the origin is necessarily unbounded near $\rho=1$. Since $\hat{u}_{1}(\rho)=\frac{1}{\left(1+\rho^{2}\right)^{2}}$ solves the homogeneous version of Eq. (5.18), another (linearly independent) solution is

$$
\hat{u}_{2}(\rho)=\hat{u}_{1}(\rho) \int_{\frac{1}{2}}^{\rho} \frac{d s}{s^{6}\left(1-s^{2}\right)^{2} \hat{u}_{1}(s)^{2}}=\frac{1}{\left(1+\rho^{2}\right)^{2}} \int_{\frac{1}{2}}^{\rho} \frac{\left(1+s^{2}\right)^{4}}{s^{6}\left(1-s^{2}\right)^{2}} d s
$$

for $\rho \in(0,1)$. Note that $\hat{u}_{2}$ is singular at both endpoints of the interval $(0,1)$. More precisely, we have $\hat{u}_{2}(\rho) \simeq \rho^{-5}$ as $\rho \rightarrow 0^{+}$and $\hat{u}_{2}(\rho) \simeq(1-\rho)^{-1}$ as $\rho \rightarrow 1^{-}$. Now, for $\rho \in(0,1)$ we have

$$
\begin{aligned}
u(\rho)=c_{1} \hat{u}_{1}(\rho)+c_{2} \hat{u}_{2}(\rho) & +\hat{u}_{2}(\rho) \int_{0}^{\rho} \hat{u}_{1}(s) H(s)\left(1-s^{2}\right) s^{6} d s \\
& -\hat{u}_{1}(\rho) \int_{0}^{\rho} \hat{u}_{2}(s) H(s)\left(1-s^{2}\right) x^{6} d s
\end{aligned}
$$

for some $c_{1}, c_{2} \in \mathbb{C}$. Boundedness of $u$ at the origin forces $c_{2}=0$ in the above expression. Note that the first and the last term on the right hand side of Eq. (5.19) are bounded near $\rho=1$. However, the remaining term is unbounded, unless the integral multiplying $\hat{u}_{2}(\rho)$ is equal to zero for $\rho=1$. This is impossible since the integrand is strictly positive on $(0,1)$. Therefore, $u(\rho) \simeq \hat{u}_{2}(\rho) \simeq(1-\rho)^{-1}$ near $\rho=1$.

Finally, for $\mathbf{Q}_{0}$,

$$
F(\xi)=\sum_{j=1}^{7} \alpha_{j}\left(2 \xi^{j} \partial_{j} q_{0,1}^{(j)}(\xi)+3 q_{0,1}^{(j)}(\xi)\right)
$$

and we have to exclude the existence of $C^{2}[0,1]$ solutions of the equation

$$
\left(1-\rho^{2}\right) u^{\prime \prime}(\rho)+\left(\frac{6}{\rho}-4 \rho\right) u^{\prime}(\rho)-\left(2+\frac{6}{\rho^{2}}-\frac{48}{\left(1+\rho^{2}\right)^{2}}\right) u(\rho)=Q(\rho)
$$

with $Q(\rho)=\frac{-\rho\left(5 \rho^{4}+6 \rho^{2}-15\right)}{\left(1+\rho^{2}\right)^{2}}$. We start with the observation that $\hat{u}_{1}(\rho)=\frac{3 \rho-\rho^{3}}{\left(1+\rho^{2}\right)^{2}}$ solves the homogeneous version of Eq. (5.20). Then another (linearly independent) solution is

$$
\hat{u}_{2}(\rho)=\hat{u}_{1}(\rho) \int_{1}^{\rho} \frac{1-s^{2}}{s^{6} \hat{u}_{1}(s)^{2}} d s .
$$

Furthermore, we have the following asymptotics, $\hat{u}_{2}(\rho) \simeq \rho^{-6}$ as $\rho \rightarrow 0^{+}$and $\hat{u}_{2}(\rho) \simeq(1-\rho)^{2}$ as $\rho \rightarrow 1^{-}$. Now, every solution to Eq. (5.20) is of the following form

$$
u(\rho)=c_{1} \hat{u}_{1}(\rho)+c_{2} \hat{u}_{2}(\rho)+\hat{u}_{2}(\rho) \int_{0}^{\rho} \frac{\hat{u}_{1}(s) Q(s) s^{6}}{\left(1-s^{2}\right)^{2}} d s-\hat{u}_{1}(\rho) \int_{0}^{\rho} \frac{\hat{u}_{2}(s) Q(s) s^{6}}{\left(1-s^{2}\right)^{2}} d s,
$$

for some complex constants $c_{1}, c_{2}$ and $\rho \in(0,1)$. Singular behavior of $\hat{u}_{2}(\rho)$ at $\rho=0$ forces $c_{2}=0$. Then simple analysis yields $u^{\prime \prime}(\rho) \simeq \ln (1-\rho)$ as $\rho \rightarrow 1^{-}$and the claim follows. 
5.2. The spectrum of $\mathbf{L}_{a}$ for $a \neq 0$. We turn to the investigation of the spectrum of $\mathbf{L}_{a}$ for small $a$.

Lemma 5.5. Let $\delta>0$ be sufficiently small. Then for all $a \in \overline{\mathbb{B}_{\delta}^{7}}$ the following holds:

$$
\sigma\left(\mathbf{L}_{a}\right) \subset\left\{\lambda \in \mathbb{C}: \operatorname{Re} \lambda<-\frac{\omega_{0}}{2}\right\} \cup\left\{\lambda_{0}, \lambda_{1}, \lambda_{2}\right\},
$$

where $\omega_{0}>0$ is the constant in Propositions 5.3 and $\lambda_{0}=0, \lambda_{1}=1, \lambda_{2}=3$ are eigenvalues. The eigenspace of $\lambda_{2}$ is one-dimensional and spanned by $\mathbf{h}_{a}=\left(h_{a, 1}, h_{a, 2}\right)$, where

$$
h_{a, 1}(\xi)=\frac{1}{\left(2 \gamma(\xi, a)^{2}+|\xi|^{2}-1\right)^{2}}, \quad h_{a, 2}(\xi)=4 h_{a, 1}(\xi)+\xi^{j} \partial_{j} h_{a, 1}(\xi) .
$$

Furthermore, the eigenspaces of $\lambda_{0}$ and $\lambda_{1}$ are spanned by $\left\{\mathbf{g}_{a}^{(k)}\right\}_{k=0, \ldots, 7}$ and $\left\{\mathbf{q}_{a}^{(j)}\right\}_{j=1, \ldots, 7}$, respectively. Explicitly, we have

$$
\begin{aligned}
g_{a, 1}^{(0)}(\xi) & =\frac{1}{2 \gamma(\xi, a)^{2}+|\xi|^{2}-1}\left(A_{0}(a) \gamma(\xi, a)^{2}-2 \frac{\gamma(\xi, a)+A_{0}(a)\left(|\xi|^{2}-1\right)}{2 \gamma(\xi, a)^{2}+|\xi|^{2}-1}\right) \\
g_{a, 2}^{(0)}(\xi) & \left.=2 g_{a, 1}^{(0)}(\xi)+\xi^{j} \partial_{j} g_{a, 1}^{(0)} \xi\right) \\
g_{a, 1}^{(j)}(\xi) & =\frac{1}{2 \gamma(\xi, a)^{2}+|\xi|^{2}-1}\left(\frac{A_{j}(a)}{\gamma(\xi, a)^{2}}+2 \frac{\xi^{j} \gamma(\xi, a)^{2}+A_{j}(a)\left(|\xi|^{2}-1\right)}{2 \gamma(\xi, a)^{2}+|\xi|^{2}-1}\right), \\
g_{a, 2}^{(j)}(\xi) & =2 g_{a, 1}^{(j)}(\xi)+\xi^{j} \partial_{j} g_{a, 1}^{(j)}(\xi), \\
q_{a, 1}^{(j)}(\xi) & =4 \partial_{a_{j}} \gamma(\xi, a) \cdot \frac{-2 \gamma(\xi, a)^{2}+|\xi|^{2}-1}{\left(2 \gamma(\xi, a)^{2}+|\xi|^{2}-1\right)^{2}} \\
q_{a, 2}^{(j)}(\xi) & =q_{a, 1}^{(j)}(\xi)+\xi^{j} \partial_{j} q_{a, 1}^{(j)}(\xi),
\end{aligned}
$$

and the eigenfunctions depend Lipschitz continuously on the parameter a, i.e.,

$$
\left\|\mathbf{h}_{a}-\mathbf{h}_{b}\right\|+\left\|\mathbf{g}_{a}^{(k)}-\mathbf{g}_{b}^{(k)}\right\|+\left\|\mathbf{q}_{a}^{(j)}-\mathbf{q}_{b}^{(j)}\right\| \lesssim|a-b|
$$

for all $a, b \in \overline{\mathbb{B}_{\delta}^{7}}$.

Proof. Let $\varepsilon=-\frac{\omega_{0}}{2}+\frac{1}{2}$, see Proposition 5.3, and let $\kappa^{*}$ be the constant associated via Lemma 5.1. We define

$$
\Omega:=\left\{\lambda \in \mathbb{C}: \operatorname{Re} \lambda \geq-\frac{\omega_{0}}{2},|\lambda| \leq \kappa^{*}\right\},
$$

and $\Omega^{\prime}:=\left\{\lambda \in \mathbb{C}: \operatorname{Re} \lambda \geq-\frac{\omega_{0}}{2}\right\} \backslash \Omega$. By Lemma 5.1, we have $\Omega^{\prime} \subset \rho\left(\mathbf{L}_{a}\right)$ and it is left to investigate the spectrum in the compact region $\Omega$. By Lemma 5.2, we know that there are only isolated eigenvalues. First, one can check by a direct calculation that $\mathbf{h}_{a}, \mathbf{g}_{a}^{(k)}, \mathbf{q}_{a}^{(j)}$ are eigenfunctions corresponding to the eigenvalues 3, 1 and 0 . The Lipschitz estimates for the eigenfunctions follow from the fact the they are smooth provided that $a$ is small enough. It is left to show that there are no other eigenvalues. First, we claim that $\partial \Omega \in \rho\left(\mathbf{L}_{a}\right)$. For this, we need information on the line segment $\Gamma:=\left\{\lambda \in \mathbb{C}: \operatorname{Re} \lambda=-\frac{\omega_{0}}{2},|\lambda| \leq \kappa^{*}\right\}$, which is contained in the resolvent set of $\mathbf{L}_{0}$ by Proposition 5.3. In view of the identity

$$
\lambda-\mathbf{L}_{a}=\left[1-\left(\mathbf{L}_{a}^{\prime}-\mathbf{L}_{0}^{\prime}\right) \mathbf{R}_{\mathbf{L}_{0}}(\lambda)\right]\left(\lambda-\mathbf{L}_{0}\right),
$$

it suffices to show that

$$
\left\|\mathbf{L}_{0}^{\prime}-\mathbf{L}_{a}^{\prime}\right\| \underset{26}{\left\|\mathbf{R}_{\mathbf{L}_{0}}(\lambda)\right\|<1}
$$


for all $\lambda \in \Gamma$ and for all $a \in \overline{\mathbb{B}_{\delta}^{7}}$ with $\delta>0$ small enough. This follows from the Lipschitz continuity of $\mathbf{L}_{a}^{\prime}$, see Lemma 3.5, if we require that $\delta<\frac{1}{2 K C}$, where $C:=\max _{\lambda \in \Gamma}\left\|\mathbf{R}_{\mathbf{L}_{0}}(\lambda)\right\|$. Having this, we define a projection

$$
\tilde{\mathbf{T}}_{\mathbf{a}}=\frac{1}{2 \pi i} \int_{\partial \Omega} \mathbf{R}_{\mathbf{L}_{a}}(\lambda) d \lambda
$$

which depends continuously on $a$ for small enough values of the parameter. This follows from the continuity of $a \mapsto \mathbf{R}_{\mathbf{L}_{a}}(\lambda)$ for small enough $a$, which can be seen for example from Eq. (5.22). For $a=0$, $\tilde{\mathbf{T}}_{\mathbf{0}}$ has rank 16, see Lemma 5.4. By [26], p. 34, Lemma 4.10, we infer that $\operatorname{dim} \operatorname{rg} \tilde{\mathbf{T}}_{\mathbf{a}}=16$ for $a$ small enough, which excludes the existence of other eigenvalues.

Remark 5.6. The eigenfunctions corresponding to the eigenvalues $\lambda=0$ and $\lambda=1$ originate from the fact that we are perturbing around a family of solutions depending on several symmetry parameters. For Lorentz boosts, this can be seen most easily. Since $\Psi_{a}^{*}$ satisfies the equation $\mathbf{L} \Psi_{a}^{*}+\mathbf{N}\left(\Psi_{a}^{*}\right)=0$, the chain rule implies that $\left(\mathbf{L}+\mathbf{N}^{\prime}\left(\Psi_{a}^{*}\right)\right) \partial_{a^{j}} \Psi_{a}^{*}=\mathbf{L}_{a} \partial_{a^{j}} \Psi_{a}^{*}=0$, i.e., $\partial_{a^{j}} \Psi_{a}^{*}$ solves the eigenvalue problem for $\lambda=0$. In fact, it can easily be checked that $\partial_{a^{j}} \Psi_{a}^{*}=\mathbf{q}_{a}^{(j)}$.

\subsection{Growth bounds for the semigroup. Define}

$$
\mathbf{H}_{a}:=\frac{1}{2 \pi i} \int_{\gamma_{2}} \mathbf{R}_{\mathbf{L}_{a}}(\lambda) d \lambda, \quad \mathbf{P}_{a}:=\frac{1}{2 \pi i} \int_{\gamma_{1}} \mathbf{R}_{\mathbf{L}_{a}}(\lambda) d \lambda, \quad \mathbf{Q}_{a}:=\frac{1}{2 \pi i} \int_{\gamma_{0}} \mathbf{R}_{\mathbf{L}_{a}}(\lambda) d \lambda,
$$

where $\gamma_{j}(s):=\lambda_{j}+\frac{\omega_{0}}{4} e^{2 \pi i s}$ for $s \in[0,1]$.

Lemma 5.7. Let $\delta>0$ be sufficiently small, then,

$$
\operatorname{rg} \mathbf{H}_{a}=\operatorname{span}\left(\mathbf{h}_{a}\right), \quad \operatorname{rg}_{\mathbf{P}_{a}}=\operatorname{span}\left(\mathbf{g}_{a}^{(0)}, \ldots, \mathbf{g}_{a}^{(7)}\right), \quad \operatorname{rg} \mathbf{Q}_{a}=\operatorname{span}\left(\mathbf{q}_{a}^{(1)}, \ldots, \mathbf{q}_{a}^{(7)}\right)
$$

for all $a \in \overline{\mathbb{B}_{\delta}^{7}}$. Furthermore, the projections are mutually transversal,

$$
\mathbf{H}_{a} \mathbf{P}_{a}=\mathbf{P}_{a} \mathbf{H}_{a}=\mathbf{H}_{a} \mathbf{Q}_{a}=\mathbf{Q}_{a} \mathbf{H}_{a}=\mathbf{Q}_{a} \mathbf{P}_{a}=\mathbf{P}_{a} \mathbf{Q}_{a}=0
$$

and depend Lipschitz continuously on the Lorentz parameter, i.e.,

$$
\left\|\mathbf{H}_{a}-\mathbf{H}_{b}\right\|+\left\|\mathbf{P}_{a}-\mathbf{P}_{b}\right\|+\left\|\mathbf{Q}_{a}-\mathbf{Q}_{b}\right\| \lesssim|a-b|
$$

for all $a, b \in \overline{\mathbb{B}_{\delta}^{7}}$.

Proof. As in the proof of Lemma 5.5, the dimension of the ranges of the projections is a consequence of the continuity of the projections with respect to the parameter $a$. Transversality follows from the definition. For the Lipschitz bounds, we use the second resolvent identity and Corollary 3.6 which imply that

$$
\left\|\mathbf{R}_{\mathbf{L}_{a}}(\lambda)-\mathbf{R}_{\mathbf{L}_{b}}(\lambda)\right\| \leq\left\|\mathbf{R}_{\mathbf{L}_{a}}(\lambda)\right\|\left\|\mathbf{L}_{a}-\mathbf{L}_{b}\right\|\left\|\mathbf{R}_{\mathbf{L}_{b}}(\lambda)\right\| \lesssim|a-b|
$$

for all $\lambda \in \operatorname{rg} \gamma_{j}$ and $a, b \in \overline{\mathbb{B}}_{\delta}^{7}$ for $\delta>0$ small enough.

Since $\mathbf{P}_{a}$ and $\mathbf{Q}_{a}$ are operators of finite rank, every $\mathbf{f} \in \mathcal{H}$ has the unique expansion

$$
\mathbf{P}_{a} \mathbf{f}=\sum_{k=0}^{7} \alpha_{k} \mathbf{g}_{a}^{(k)}, \quad \mathbf{Q}_{a} \mathbf{f}=\sum_{j=1}^{7} \beta_{j} \mathbf{q}_{a}^{(j)},
$$


for $\alpha_{k}, \beta_{j} \in \mathbb{C}$. We define

$$
\mathbf{P}_{a}^{(k)} \mathbf{f}:=\alpha_{k} \mathbf{g}_{a}^{(k)}, \quad \mathbf{Q}_{a}^{(j)} \mathbf{f}:=\beta_{j} \mathbf{q}_{a}^{(j)},
$$

such that $\mathbf{P}_{a}=\sum_{k=0}^{7} \mathbf{P}_{a}^{(k)}, \mathbf{Q}_{a}=\sum_{j=1}^{7} \mathbf{Q}_{a}^{(j)}$ and

$$
\mathbf{P}_{a}^{(k)} \mathbf{P}_{a}^{(l)}=\delta_{k l} \mathbf{P}_{a}^{(k)}, \quad \mathbf{Q}_{a}^{(i)} \mathbf{Q}_{a}^{(j)}=\delta_{i j} \mathbf{Q}_{a}^{(j)} .
$$

Finally, we define

$$
\mathbf{T}_{a}:=\mathbf{I}-\mathbf{H}_{a}-\mathbf{P}_{a}-\mathbf{Q}_{a},
$$

which is Lipschitz continuous with respect to $a$ by Lemma 5.7. Note that the projections $\mathbf{T}_{a}, \mathbf{H}_{a}, \mathbf{P}_{a}^{(k)}$ and $\mathbf{Q}_{a}^{(j)}$ are mutually transversal. Furthermore, is easy to see that the Lipschitz continuity of $\mathbf{Q}_{a}$ and the eigenfunctions $\mathbf{q}_{a}^{(j)}, j=1, \ldots, 7$ imply that

$$
\left\|\mathbf{Q}_{a}^{(j)}-\mathbf{Q}_{b}^{(j)}\right\| \lesssim|a-b|
$$

for all $a, b \in \overline{\mathbb{B}_{\delta}^{7}}$. Similarly,

$$
\left\|\mathbf{P}_{a}^{(k)}-\mathbf{P}_{b}^{(k)}\right\| \lesssim|a-b|
$$

for $k=0, \ldots, 7$.

Theorem 5.8. The projections commute with the semigroup,

$$
\left[\mathbf{S}_{a}(\tau), \mathbf{H}_{a}\right]=\left[\mathbf{S}_{a}(\tau), \mathbf{P}_{a}^{(k)}\right]=\left[\mathbf{S}_{a}(\tau), \mathbf{Q}_{a}^{(j)}\right]=0,
$$

and there are constants $\delta>0$ and $\omega>0$ such that

$$
\mathbf{S}_{a}(\tau) \mathbf{H}_{a}=e^{3 \tau} \mathbf{H}_{a}, \quad \mathbf{S}_{a}(\tau) \mathbf{P}_{a}^{(k)}=e^{\tau} \mathbf{P}_{a}^{(k)}, \quad \mathbf{S}_{a}(\tau) \mathbf{Q}_{a}^{(j)}=\mathbf{Q}_{a}^{(j)},
$$

and

$$
\left\|\mathbf{S}_{a}(\tau) \mathbf{T}_{a} \mathbf{u}\right\| \lesssim e^{-\omega \tau}\left\|\mathbf{T}_{a} \mathbf{u}\right\|
$$

for all $\tau \geq 0, \mathbf{u} \in \mathcal{H}, a \in \overline{\mathbb{B}_{\delta}^{7}}$. Furthermore, we have

$$
\left\|\mathbf{S}_{a}(\tau) \mathbf{T}_{a}-\mathbf{S}_{b}(\tau) \mathbf{T}_{b}\right\| \lesssim e^{-\omega \tau}|a-b|,
$$

for all $a, b \in \overline{\mathbb{B}_{\delta}^{7}}$ and all $\tau \geq 0$.

Proof. For the semigroup acting on the ranges of the projections $\mathbf{H}_{a}, \mathbf{P}_{a}$ and $\mathbf{Q}_{a}$ we have

$$
\mathbf{S}_{a}(\tau) \mathbf{H}_{a} \mathbf{u}=e^{3 \tau} \mathbf{H}_{a} \mathbf{u}, \quad \mathbf{S}_{a}(\tau) \mathbf{P}_{a} \mathbf{u}=e^{\tau} \mathbf{P}_{a} \mathbf{u}, \quad \mathbf{S}_{a}(\tau) \mathbf{Q}_{a} \mathbf{u}=\mathbf{Q}_{a} \mathbf{u},
$$

for all $\mathbf{u} \in \mathcal{H}, \tau \geq 0$ and sufficiently small $a$. Furthermore, the semigroup commutes with the resolvent and therefore with the projection operators,

$$
\left[\mathbf{S}_{a}(\tau), \mathbf{H}_{a}\right]=\left[\mathbf{S}_{a}(\tau), \mathbf{P}_{a}\right]=\left[\mathbf{S}_{a}(\tau), \mathbf{Q}_{a}\right]=0 .
$$

This implies that for $k=0, \ldots, 7$, we have

$$
\mathbf{P}_{a}^{(k)} \mathbf{S}_{a}(\tau) \mathbf{u}=\mathbf{P}_{a} \mathbf{P}_{a}^{(k)} \mathbf{S}_{a}(\tau) \mathbf{u}=\mathbf{P}_{a}^{(k)} \mathbf{S}_{a}(\tau) \mathbf{P}_{a} \mathbf{u}=e^{\tau} \mathbf{P}_{a}^{(k)} \mathbf{P}_{a} \mathbf{u}=\mathbf{S}_{a}(\tau) \mathbf{P}_{a}^{(k)} \mathbf{u} .
$$

The argument for $\mathbf{Q}_{a}^{(j)}$ is analogous. Now, $\mathbf{R}_{\mathbf{L}_{a}}(\lambda) \mathbf{T}_{a}$ is holomorphic in $\Omega$, see Eq. (5.21), and uniformly bounded with respect to $a \in \overline{\mathbb{B}_{\delta}^{7}}$. In view of Lemma 5.1, we infer that there is a constant $c>0$ such that

$$
\left\|\mathbf{R}_{\mathbf{L}_{a}}(\lambda) \mathbf{T}_{a}\right\| \leq c
$$


for all $\lambda \in \mathbb{C}$ with $\operatorname{Re} \lambda \geq-\frac{\omega_{0}}{2}$ and all $a \in \overline{\mathbb{B}_{\delta}^{7}}$. An application of the Gearhart-Prüss Theorem, see [37], Proposition 2, shows that for every $\varepsilon>0$, there exists a constant $C_{\varepsilon}$ such that

$$
\left\|\mathbf{S}_{a}(\tau) \mathbf{T}_{a} \mathbf{u}\right\| \leq C_{\varepsilon} e^{-\left(\frac{\omega_{0}}{2}-\varepsilon\right) \tau}\left\|\mathbf{T}_{a} \mathbf{u}\right\|
$$

for all $\mathbf{u} \in \mathcal{H}$ and all $a \in \overline{\mathbb{B}_{\delta}^{7}}$. Eq. (5.25) is obtained analogously to [18], Lemma 3.9. One can easily check that for $\mathbf{u} \in \mathcal{D}\left(\mathbf{L}_{a}\right)$, the function

$$
\Phi_{a, b}(\tau):=\frac{\mathbf{S}_{a}(\tau) \mathbf{T}_{a} \mathbf{u}-\mathbf{S}_{b}(\tau) \mathbf{T}_{b} \mathbf{u}}{|a-b|}
$$

satisfies the inhomogeneous equation

$$
\partial_{\tau} \Phi_{a, b}(\tau)=\mathbf{L}_{a} \mathbf{T}_{a} \Phi_{a, b}(\tau)+\frac{\mathbf{L}_{a} \mathbf{T}_{a}-\mathbf{L}_{b} \mathbf{T}_{b}}{|a-b|} \mathbf{S}_{b}(\tau) \mathbf{T}_{b} \mathbf{u}
$$

with initial data $\Phi_{a, b}(0)=\frac{\mathbf{T}_{a} \mathbf{u}-\mathbf{T}_{b} \mathbf{u}}{|a-b|}$ for all $\tau \geq 0$. We have $\mathbf{L}_{a} \mathbf{T}_{a}=\mathbf{L}_{a}\left(1-\mathbf{H}_{a}-\mathbf{P}_{a}-\mathbf{Q}_{a}\right)=$ $\mathbf{L}_{a}-3 \mathbf{H}_{a}-\mathbf{P}_{a}$, such that

$$
\mathbf{L}_{a} \mathbf{T}_{a}-\mathbf{L}_{b} \mathbf{T}_{b}=\mathbf{L}_{a}^{\prime}-\mathbf{L}_{b}^{\prime}-3\left(\mathbf{H}_{a}-\mathbf{H}_{b}\right)-\left(\mathbf{P}_{a}-\mathbf{P}_{b}\right)
$$

which implies that

$$
\left\|\mathbf{L}_{a} \mathbf{T}_{a}-\mathbf{L}_{b} \mathbf{T}_{b}\right\| \lesssim|a-b|
$$

by Lemma 3.5 and Lemma 5.7. The integral equation associated to Eq. (5.27) by the Duhamel principle is given by

$$
\Phi_{a, b}(\tau)=\mathbf{S}_{a}(\tau) \mathbf{T}_{a} \frac{\mathbf{T}_{a} \mathbf{u}-\mathbf{T}_{b} \mathbf{u}}{|a-b|}+\int_{0}^{\tau} \mathbf{S}_{a}\left(\tau-\tau^{\prime}\right) \mathbf{T}_{a} \frac{\mathbf{L}_{a} \mathbf{T}_{a}-\mathbf{L}_{b} \mathbf{T}_{b}}{|a-b|} \mathbf{S}_{b}\left(\tau^{\prime}\right) \mathbf{T}_{b} \mathbf{u} d \tau^{\prime}
$$

Eq. (5.26) yields the bound

$$
\left\|\Phi_{a, b}(\tau)\right\| \lesssim e^{-\left(\frac{\omega_{0}}{2}-\varepsilon\right) \tau}(1+\tau)\|\mathbf{u}\| \lesssim e^{-\left(\frac{\omega_{0}}{2}-2 \varepsilon\right) \tau}\|\mathbf{u}\|,
$$

which extends to all of $\mathcal{H}$ by density. We fix $\varepsilon>0$ such that $\omega:=\frac{\omega_{0}}{2}-2 \varepsilon>0$. This yields Eq. (5.25) and Eq. (5.24).

\section{Nonlinear PERTURBation theOry}

6.1. Function spaces and basic estimates. Let $\omega>0$ be fixed by Theorem 5.8. We define spaces

$$
\begin{gathered}
\mathcal{X}:=\left\{\Phi \in C([0, \infty), \mathcal{H}):\|\Phi\|_{\mathcal{X}}<\infty\right\}, \quad\|\Phi\|_{\mathcal{X}}:=\sup _{\tau>0} e^{\omega \tau}\|\Phi(\tau)\|, \\
X:=\left\{a \in C^{1}\left([0, \infty), \mathbb{R}^{7}\right): a(0)=0,\|a\|_{X}<\infty\right\}, \quad\|a\|_{X}:=\sup _{\tau>0}\left[e^{\omega \tau}|\dot{a}(\tau)|+|a(\tau)|\right] .
\end{gathered}
$$

For $a \in X$, we can write $a(\tau)=\int_{0}^{\tau} \dot{a}(\sigma) d \sigma$ and the integral converges in the limit $\tau \rightarrow \infty$. Hence, we define

$$
a_{\infty}=\lim _{\tau \rightarrow \infty} a(\tau)
$$

In the following, we assume that $\tilde{\delta}>0$ is sufficiently small, such that the results of the preceding sections hold for all $a \in \overline{\mathbb{B}_{\tilde{\delta}}^{7}}$. For $\delta>0$ satisfying $\frac{\delta}{\omega}<\tilde{\delta}$, we set

$$
\mathcal{X}_{\delta}:=\{\Phi \in \mathcal{X}:\|\Phi\| \leq \delta\}, \quad X_{\delta}:=\left\{a \in X: \sup _{\tau>0}\left[e^{\omega \tau}|\dot{a}(\tau)|\right] \leq \delta\right\} .
$$


For $a \in X_{\delta},|a(\tau)| \leq \delta / \omega<\tilde{\delta}$ for all $\tau \geq 0$. In the following, we will frequently use that

$$
\left|a_{\infty}-a(\tau)\right| \leq \int_{\tau}^{\infty}|\dot{a}(\sigma)| d \sigma \leq \frac{\delta}{\omega} e^{-\omega \tau},
$$

and $|a(\tau)-b(\tau)| \leq\|a-b\|_{X}$ for all $a, b \in X_{\delta}$ and all $\tau \geq 0$. In particular, $\left|a_{\infty}-b_{\infty}\right| \leq\|a-b\|_{X}$. In the following, we provide some bounds for the function $\mathbf{G}_{a}$ defined by

$$
\mathbf{G}_{a(\tau)}(\Phi(\tau))=\left[\mathbf{L}_{a(\tau)}^{\prime}-\mathbf{L}_{a_{\infty}}^{\prime}\right] \Phi(\tau)+\mathbf{F}_{a(\tau)}(\Phi(\tau))
$$

with $\mathbf{F}_{a}$ given in Eq. (2.7). We start with some basic estimates for the nonlinear part and denote by $\mathcal{B} \subset \mathcal{H}$ the unit ball in $\mathcal{H}$.

Lemma 6.1. Let $\delta>0$ be sufficiently small. Then

$$
\left\|\mathbf{F}_{a}(\mathbf{u})-\mathbf{F}_{b}(\mathbf{v})\right\| \lesssim(\|\mathbf{u}\|+\|\mathbf{v}\|)\|\mathbf{u}-\mathbf{v}\|+\left(\|\mathbf{u}\|^{2}+\|\mathbf{v}\|^{2}\right)|a-b|
$$

for all $\mathbf{u}, \mathbf{v} \in \mathcal{B} \subset \mathcal{H}$ and all $a, b \in \overline{\mathbb{B}_{\delta}^{7}}$.

Proof. First, we show that

$$
\left\|\mathbf{F}_{a}(\mathbf{u})-\mathbf{F}_{a}(\mathbf{v})\right\| \lesssim(\|\mathbf{u}\|+\|\mathbf{v}\|)\|\mathbf{u}-\mathbf{v}\|
$$

for all $a \in \overline{\mathbb{B}_{\delta}^{7}}$ and all $\mathbf{u}, \mathbf{v} \in \mathcal{B}$, more precisely, we prove that

$$
\left\|u^{3}-v^{3}\right\|_{H^{2}\left(\mathbb{B}^{7}\right)}+\left\|\psi_{a}^{*}\left(u^{2}-v^{2}\right)\right\|_{H^{2}\left(\mathbb{B}^{7}\right)} \lesssim\left(\|u\|_{H^{3}\left(\mathbb{B}^{7}\right)}+\|v\|_{H^{3}\left(\mathbb{B}^{7}\right)}\right)\|u-v\|_{H^{3}\left(\mathbb{B}^{7}\right)}
$$

for all $u, v \in H^{3}\left(\mathbb{B}^{7}\right)$. The Sobolev embedding $W^{j+m, 2}\left(\mathbb{B}^{d}\right) \hookrightarrow W^{j, q}\left(\mathbb{B}^{d}\right)$ for $2 \leq q \leq \frac{2 d}{d-2 m}$, $j \in \mathbb{N}_{0}$, implies that

$$
\left\|\partial^{\alpha} u\right\|_{L^{q}\left(\mathbb{B}^{7}\right)} \lesssim\|u\|_{H^{3}\left(\mathbb{B}^{7}\right)}
$$

for multi-indices $\alpha \in \mathbb{N}^{7}$ with $0 \leq|\alpha| \leq 2$ and $2<q \leq \frac{14}{1+2|\alpha|}$. Using this, we show that

$$
\|u v w\|_{H^{2}\left(\mathbb{B}^{7}\right)} \lesssim\|u\|_{H^{3}\left(\mathbb{B}^{7}\right)}\|v\|_{H^{3}\left(\mathbb{B}^{7}\right)}\|w\|_{H^{3}\left(\mathbb{B}^{7}\right)}
$$

for all $u, v, w \in H^{3}\left(\mathbb{B}^{7}\right)$. First, observe that Hölder's inequality with $q_{1}=\frac{14}{5}, q_{2}=14$, $\frac{1}{q_{1}}+\frac{2}{q_{2}}=\frac{1}{2}$ implies

$$
\|u v w\|_{L^{2}\left(\mathbb{B}^{7}\right)} \lesssim\|u\|_{L^{q_{1}\left(\mathbb{B}^{7}\right)}}\|v\|_{L^{q_{2}\left(\mathbb{B}^{7}\right)}}\|w\|_{L^{q_{2}\left(\mathbb{B}^{7}\right)}} \lesssim\|u\|_{H^{3}\left(\mathbb{B}^{7}\right)}\|v\|_{H^{3}\left(\mathbb{B}^{7}\right)}\|w\|_{H^{3}\left(\mathbb{B}^{7}\right)} .
$$

For $|\alpha|=1$ we have

$$
\left\|v w \partial^{\alpha} u\right\|_{L^{2}\left(\mathbb{B}^{7}\right)} \lesssim\left\|\partial^{\alpha} u\right\|_{L^{q_{1}\left(\mathbb{B}^{7}\right)}}\|v\|_{L^{q_{2}\left(\mathbb{B}^{7}\right)}}\|w\|_{L^{q_{2}\left(\mathbb{B}^{7}\right)}} \lesssim\|u\|_{H^{3}\left(\mathbb{B}^{7}\right)}\|v\|_{H^{3}\left(\mathbb{B}^{7}\right)}\|w\|_{H^{3}\left(\mathbb{B}^{7}\right)},
$$

where $q_{1}=\frac{14}{3}, q_{2}=7$. For $|\alpha|=2, \alpha_{j} \in \mathbb{N}^{7}, j=1,2,3$, with $\sum_{j} \alpha_{j}=\alpha$ we set $q_{j}=\frac{14}{1+2\left|\alpha_{j}\right|}$ to obtain

$\left\|\partial^{\alpha_{1}} u \partial^{\alpha_{2}} v \partial^{\alpha_{3}} w\right\|_{L^{2}\left(\mathbb{B}^{7}\right)} \lesssim\left\|\partial^{\alpha_{1}} u\right\|_{L^{q_{1}\left(\mathbb{B}^{7}\right)}}\left\|\partial^{\alpha_{2}} v\right\|_{L^{q_{2}\left(\mathbb{B}^{7}\right)}}\left\|\partial^{\alpha_{3}} w\right\|_{L^{q_{3}\left(\mathbb{B}^{7}\right)}} \lesssim\|u\|_{H^{3}}\|v\|_{H^{3}\left(\mathbb{B}^{7}\right)}\|w\|_{H^{3}\left(\mathbb{B}^{7}\right)}$.

Eq. (6.2) now follows by applying the Leibnitz rule. Consequently,

$$
\left\|u^{3}-v^{3}\right\|_{H^{2}\left(\mathbb{B}^{7}\right)} \lesssim\left(\|u\|_{H^{3}\left(\mathbb{B}^{7}\right)}^{2}+\|v\|_{H^{3}\left(\mathbb{B}^{7}\right)}^{2}\right)\|u-v\|_{H^{3}\left(\mathbb{B}^{7}\right)} .
$$

Since $\psi_{a}^{*}$ is smooth and uniformly bounded for sufficiently small $a$, we obtain

$$
\left\|\psi_{a}^{*}\left(u^{2}-v^{2}\right)\right\|_{H^{2}\left(\mathbb{B}^{7}\right)} \lesssim\left(\|u\|_{H^{3}\left(\mathbb{B}^{7}\right)}+\|v\|_{H^{3}\left(\mathbb{B}^{7}\right)}\right)\|u-v\|_{H^{3}\left(\mathbb{B}^{7}\right)} .
$$

Finally, in view of Eqns. (6.2) and (2.3) we have

$$
\left\|\mathbf{F}_{a}(\mathbf{u})-\mathbf{F}_{b}(\mathbf{u})\right\| \lesssim\left\|u^{2}\left(\psi_{a}^{*}-\psi_{b}^{*}\right)\right\|_{H^{2}\left(\mathbb{B}^{7}\right)} \lesssim\|u\|_{H^{3}\left(\mathbb{B}^{7}\right)}^{2}\left\|\psi_{a}^{*}-\psi_{b}^{*}\right\|_{H^{3}\left(\mathbb{B}^{7}\right)} \lesssim\|u\|_{H^{3}\left(\mathbb{B}^{7}\right)}^{2}|a-b|,
$$


for all $a, b \in \overline{\mathbb{B}_{\delta}^{7}}$ which implies the claim.

Lemma 6.2. Let $\delta>0$ be sufficiently small. Then

$$
\begin{gathered}
\left\|\mathbf{G}_{a(\tau)}(\Phi(\tau))\right\| \lesssim \delta^{2} e^{-2 \omega \tau} \\
\left\|\mathbf{G}_{a(\tau)}(\Phi(\tau))-\mathbf{G}_{b(\tau)}(\Psi(\tau))\right\| \lesssim \delta e^{-2 \omega \tau}\left(\|\Phi-\Psi\|_{\mathcal{X}}+\|a-b\|_{X}\right),
\end{gathered}
$$

for all $\Phi, \Psi \in \mathcal{X}_{\delta}, a, b \in X_{\delta}$, and $\tau \geq 0$.

Proof. Since $\mathbf{F}_{a}(0)=0$ for all $a$, Lemma 6.1, Lemma 3.5 and Eq. (6.1) immediately imply the first estimate. For the Lipschitz bounds, we obtain

$$
\left\|\mathbf{F}_{a(\tau)}(\Phi(\tau))-\mathbf{F}_{b(\tau)}(\Psi(\tau))\right\| \lesssim \delta e^{-2 \omega \tau}\left(\|\Phi-\Psi\|_{\mathcal{X}}+\|a-b\|_{X}\right)
$$

from Lemma 6.1. Furthermore,

$$
\left\|\left[\mathbf{L}_{b(\tau)}^{\prime}-\mathbf{L}_{b_{\infty}}^{\prime}\right](\Phi(\tau)-\Psi(\tau))\right\| \lesssim \delta e^{-2 \omega \tau}\|\Phi-\Psi\|_{\mathcal{X}}
$$

by Lemma 3.5 and Eq. (6.1). Finally, we use that

$$
\psi_{a_{\infty}}^{*}(\xi)^{2}-\psi_{a(\tau)}^{*}(\xi)^{2}=\int_{\tau}^{\infty} \dot{a}^{k}(s) \varphi_{a(s), k}(\xi) d s
$$

with $\varphi_{a, k}(\xi)=\partial_{a^{k}} \psi_{a}^{*}(\xi)^{2}$, to obtain the estimate

$$
\left\|\left[\mathbf{L}_{a(\tau)}^{\prime}-\mathbf{L}_{a_{\infty}}^{\prime}\right]-\left[\mathbf{L}_{b(\tau)}^{\prime}-\mathbf{L}_{b_{\infty}}^{\prime}\right]\right\| \lesssim e^{-\omega \tau}\|a-b\|_{X},
$$

see the proof of Lemma 5.5 in [18] for the details. These bounds imply the second line in Eq. (6.3).

6.2. Integral equation for the peturbation. To solve Eq. (2.9), we first study the general initial value problem,

$$
\begin{aligned}
\partial_{\tau} \Phi(\tau) & =\mathbf{L}_{a_{\infty}} \Phi(\tau)+\mathbf{G}_{a(\tau)}(\Phi(\tau))-\partial_{\tau} \Psi_{a(\tau)}^{*}, \quad \tau>0 \\
\Phi(0) & =\mathbf{u}
\end{aligned}
$$

for $\mathbf{u} \in \mathcal{H}$. In fact, we are interested in solutions of the corresponding integral equation

$$
\Phi(\tau)=\mathbf{S}_{a_{\infty}}(\tau) \mathbf{u}+\int_{0}^{\tau} \mathbf{S}_{a_{\infty}}(\tau-\sigma)\left[\mathbf{G}_{a(\sigma)}(\Phi(\sigma))-\partial_{\sigma} \Psi_{a(\sigma)}^{*}\right] d \sigma .
$$

In the following, we use modulation theory to prove the existence of solutions in $\mathcal{X}_{\delta}$ under a co-dimension 9 condition on the initial data $\mathbf{u} \in \mathcal{H}$ (corresponding to the unstable directions defined by $\mathbf{g}_{a}^{(k)}, k=0, \ldots, 7$ and the genuine unstable mode $\mathbf{h}_{a}$ ). This is along the lines of [18], Section 5.2 - Section 5.4. In Section 6.3 below we use the specific form of the initial data in Eq. (2.9) to remove the translation instabilities by fixing suitable parameters $\left(T, x_{0}\right)$ and to prove co-dimension one stability. 
6.2.1. The modulation equation. First, we take care of the Lorentz instability by deriving a suitable modulation equation for the parameter $a$. Here, it is crucial that $\partial_{\tau} \Psi_{a(\tau)}^{*}=$ $\dot{a}_{j}(\tau) \mathbf{q}_{a(\tau)}^{(j)}=\sum_{j=1}^{7} \dot{a}^{j}(\tau) \mathbf{q}_{a(\tau)}^{(j)}$, see Remark 5.6.

We introduce a cut-off function $\chi:[0, \infty) \rightarrow[0,1]$ satisfying $\chi(\tau)=1$ for $\tau \in[0,1]$, $\left|\chi^{\prime}(\tau)\right| \leq 1$ for all $\tau \geq 0$ and $\chi(\tau)=0$ for $\tau \geq 4$, and require that

$$
\mathbf{Q}_{a_{\infty}}^{(j)} \Phi(\tau)=\chi(\tau) \mathbf{Q}_{a_{\infty}}^{(j)} \mathbf{u}
$$

for all $\tau \geq 0$. Applying $\mathbf{Q}_{a_{\infty}}^{(j)}$ to Eq. (6.5) and using Theorem 5.8 yields

$$
[1-\chi(\tau)] \mathbf{Q}_{a_{\infty}}^{(j)} \mathbf{u}+\int_{0}^{\tau}\left[\mathbf{Q}_{a_{\infty}}^{(j)} \mathbf{G}_{a(\sigma)}(\Phi(\sigma))-\mathbf{Q}_{a_{\infty}}^{(j)} \dot{a}_{i}(\sigma) \mathbf{q}_{a(\sigma)}^{(i)}\right] d \sigma=0 .
$$

In view of $\mathbf{Q}_{a_{\infty}}^{(j)} \mathbf{q}_{a_{\infty}}^{(i)}=\delta^{i j} \mathbf{q}_{a_{\infty}}^{(j)}$ and $a(0)=0$ this can be written as

$$
\begin{aligned}
a^{j}(\tau) \mathbf{q}_{a_{\infty}}^{(j)} & =-\int_{0}^{\tau} \chi^{\prime}(\sigma) \mathbf{Q}_{a_{\infty}}^{(j)} \mathbf{u} d \sigma+\int_{0}^{\tau}\left[\mathbf{Q}_{a_{\infty}}^{(j)} \mathbf{G}_{a(\sigma)}(\Phi(\sigma))-\mathbf{Q}_{a_{\infty}}^{(j)} \dot{a}_{i}(\sigma)\left[\mathbf{q}_{a(\sigma)}^{(i)}-\mathbf{q}_{a_{\infty}}^{(i)}\right]\right] d \sigma \\
& =: \int_{0}^{\tau} \mathbf{A}_{j}(a, \Phi, \mathbf{u})(\sigma) d \sigma .
\end{aligned}
$$

Thus, we obtain the equation

$$
a(\tau)=A(a, \Phi, \mathbf{u})(\tau)
$$

for $a \in X_{\delta}$, where $A=\left(A_{1}, \ldots, A_{7}\right)$, and

$$
A_{j}(a, \Phi, \mathbf{u})(\tau):=\left\|\mathbf{q}_{a_{\infty}}^{(j)}\right\|^{-2} \int_{0}^{\tau}\left(\mathbf{A}_{j}(a, \Phi, \mathbf{u})(\sigma) \mid \mathbf{q}_{a_{\infty}}^{(j)}\right) d \sigma .
$$

Lemma 6.3. Let $\delta>0$ be sufficiently small and $c>0$ be sufficiently large. For every $\mathbf{u} \in \mathcal{H}$ satisfying $\|\mathbf{u}\| \leq \frac{\delta}{c}$ and every $\Phi \in \mathcal{X}_{\delta}$, there is a unique $a=a(\Phi, \mathbf{u}) \in X_{\delta}$ such that Eq. (6.7) holds. Furthermore,

$$
\|a(\Phi, \mathbf{u})-a(\Psi, \mathbf{v})\|_{X} \lesssim\|\Phi-\Psi\|_{\mathcal{X}}+\|\mathbf{u}-\mathbf{v}\|
$$

for all $\Psi, \Phi \in \mathcal{X}_{\delta}$ and $\mathbf{u}, \mathbf{v} \in \mathcal{B}_{\delta / c}$.

Proof. We use a fixed point argument and show that under the above assumptions $A(\cdot, \Phi, \mathbf{u})$ : $X_{\delta} \rightarrow X_{\delta}$ defines a contraction. We have

$$
\left\|\mathbf{A}_{j}(a, \Phi, \mathbf{u})(\tau)\right\| \lesssim\left(\frac{\delta}{c}+\delta^{2}\right) e^{-2 \omega \tau}
$$

provided $\delta>0$ is sufficiently small and $c>0$ is sufficiently large. This can be seen by using the bounds of Lemma 6.2 , the fact that $\left\|\chi^{\prime}(\tau) \mathbf{Q}_{a_{\infty}}^{(j)} \mathbf{u}\right\| \lesssim \frac{\delta}{c} e^{-2 \omega \tau}$ and

$$
\left\|\dot{a}^{j}(\tau)\left[\mathbf{q}_{a(\tau)}^{(j)}-\mathbf{q}_{a_{\infty}}^{(j)}\right]\right\| \lesssim \delta e^{-\omega \tau}\left|a(\tau)-a_{\infty}\right| \lesssim \delta^{2} e^{-2 \omega \tau} .
$$

This implies that $|\dot{A}(a, \Phi, \mathbf{u})(\tau)| \leq \delta e^{-2 \omega \tau}$ and hence $A(\cdot, \Phi, \mathbf{u}): X_{\delta} \rightarrow X_{\delta}$. Next, we show that

$$
\left\|\mathbf{A}_{j}(a, \Phi, \mathbf{u})(\tau)-\mathbf{A}_{j}(b, \Phi, \mathbf{u})(\tau)\right\| \lesssim \delta e^{-2 \omega \tau}\|a-b\|_{X}
$$

for all $a, b, \in X_{\delta}$, which implies that

$$
\|A(a, \Phi, \mathbf{u})-A(b, \Phi, \mathbf{u})\|_{X} \lesssim \delta\|a-b\|_{X}
$$


by using the Lipschitz continuity of $a \mapsto \mathbf{q}_{a}^{(j)}$. To prove Eq. (6.8) we use the Lipschitz bounds of Lemma 6.2 and Eq. (5.23) to obtain

$$
\left\|\mathbf{Q}_{a_{\infty}}^{(j)} \mathbf{G}_{a(\tau)}(\Phi(\tau))-\mathbf{Q}_{b_{\infty}}^{(j)} \mathbf{G}_{b(\tau)}(\Psi(\tau))\right\| \lesssim \delta e^{-2 \omega \tau}\left(\|a-b\|_{X}+\|\Phi-\Psi\|_{\mathcal{X}}\right)
$$

and $\left\|\chi^{\prime}(\tau)\left[\mathbf{Q}_{a_{\infty}}^{(j)}-\mathbf{Q}_{b_{\infty}}^{(j)}\right] \mathbf{u}\right\| \lesssim \delta e^{-2 \omega \tau}\|a-b\|_{X}$. Furthermore, we have

$$
\left\|\mathbf{q}_{a(\tau)}^{(j)}-\mathbf{q}_{a_{\infty}}^{(j)}\right\| \lesssim \delta e^{-\omega \tau}
$$

and

$$
\left\|\left(\mathbf{q}_{a(\tau)}^{(j)}-\mathbf{q}_{a \infty}^{(j)}\right)-\left(\mathbf{q}_{b(\tau)}^{(j)}-\mathbf{q}_{b_{\infty}}^{(j)}\right)\right\| \lesssim e^{-\omega \tau}\|a-b\|_{X} .
$$

The last estimate is a consequence of the fact that

$$
\mathbf{q}_{a(\tau)}^{(j)}(\xi)-\mathbf{q}_{a_{\infty}}^{(j)}(\xi)=-\int_{\tau}^{\infty} \dot{a}^{i}(\sigma) \varphi_{i}^{(j)}(\xi, a(\sigma)) d \sigma
$$

where $\varphi_{i}^{(j)}(\xi, a)=\partial_{a^{i}} \mathbf{q}_{a}^{(j)}(\xi)$ is smooth with respect to both variables for small enough $a$. Thus,

$$
\left\|\mathbf{Q}_{a_{\infty}}^{(j)} \dot{a}^{i}(\tau)\left(\mathbf{q}_{a(\tau)}^{(i)}-\mathbf{q}_{a_{\infty}}^{(i)}\right)-\mathbf{Q}_{b_{\infty}}^{(j)} \dot{b}^{i}(\tau)\left(\mathbf{q}_{b(\tau)}^{(i)}-\mathbf{q}_{b_{\infty}}^{(i)}\right)\right\| \lesssim \delta e^{-2 \omega \tau}\|a-b\|_{X} .
$$

By combining these estimates, we obtain Eq. (6.8). We conclude that Eq. (6.7) has a unique fixed point in $X_{\delta}$ for $\delta>0$ sufficiently small. It is left to show the Lipschitz continuity of the solution map. Let $a=A(a, \Phi, \mathbf{u}), b=A(b, \Psi, \mathbf{v})$. It is easy to see that

$$
\|A(b, \Phi, \mathbf{u})-A(b, \Phi, \mathbf{v})\|_{X} \lesssim\|\mathbf{u}-\mathbf{v}\| .
$$

and $\|A(b, \Phi, \mathbf{v})-A(b, \Psi, \mathbf{v})\|_{X} \lesssim \delta\|\Phi-\Psi\|_{\mathcal{X}}$. By combining these bounds with Eq. (6.9) we infer that

$$
\begin{aligned}
\|a-b\|_{X} & \leq\|A(a, \Phi, \mathbf{u})-A(b, \Phi, \mathbf{u})\|_{X}+\|A(b, \Phi, \mathbf{u})-A(b, \Phi, \mathbf{v})\|_{X} \\
& +\|A(b, \Phi, \mathbf{v})-A(b, \Psi, \mathbf{v})\|_{X} \lesssim \delta\|a-b\|_{X}+\|\mathbf{u}-\mathbf{v}\|+\delta\|\Phi-\Psi\|_{\mathcal{X}}
\end{aligned}
$$

The claim follows by choosing $\delta>0$ sufficiently small.

6.2.2. Global existence for modified initial data. To control the remaining instabilities we define correction terms

$$
\begin{aligned}
& \mathbf{C}_{1}(\Phi, a, \mathbf{u}):=\mathbf{P}_{a_{\infty}} \mathbf{u}+\mathbf{P}_{a_{\infty}} \int_{0}^{\infty} e^{-\sigma}\left[\mathbf{G}_{a(\sigma)}(\Phi(\sigma))-\partial_{\sigma} \Psi_{a(\sigma)}^{*}\right] d \sigma \\
& \mathbf{C}_{2}(\Phi, a, \mathbf{u}):=\mathbf{H}_{a_{\infty}} \mathbf{u}+\mathbf{H}_{a_{\infty}} \int_{0}^{\infty} e^{-3 \sigma}\left[\mathbf{G}_{a(\sigma)}(\Phi(\sigma))-\partial_{\sigma} \Psi_{a(\sigma)}^{*}\right] d \sigma
\end{aligned}
$$

and set $\mathbf{C}:=\mathbf{C}_{1}+\mathbf{C}_{2}$. In the following, we prove that Eq. (6.4) with modified initial data $\Phi(0)=\mathbf{u}-\mathbf{C}(\Phi, a, \mathbf{u})$ has a solution in $\mathcal{X}$. For this we study the integral equation

$$
\begin{aligned}
\Phi(\tau) & =\mathbf{S}_{a_{\infty}}(\tau)[\mathbf{u}-\mathbf{C}(\Phi, a, \mathbf{u})]+\int_{0}^{\tau} \mathbf{S}_{a_{\infty}}(\tau-\sigma)\left[\mathbf{G}_{a(\sigma)}(\Phi(\sigma))-\partial_{\sigma} \Psi_{a(\sigma)}^{*}\right] d \sigma \\
& =: \mathbf{K}(\Phi, a, \mathbf{u})(\tau)
\end{aligned}
$$


Proposition 6.4. Let $c>0$ be sufficiently large and let $\delta>0$ be sufficiently small. If $\|\mathbf{u}\| \leq \frac{\delta}{c}$ then there exist functions $\Phi \in \mathcal{X}_{\delta}$ and $a \in X_{\delta}$ such that Eq. (6.12) holds for all $\tau \geq 0$. Furthermore, the map $\mathbf{u} \mapsto(\Phi(\mathbf{u}), a(\mathbf{u}))$ is Lipschitz continuous, i.e.,

$$
\|\Phi(\mathbf{u})-\Phi(\mathbf{v})\|_{\mathcal{X}}+\|a(\mathbf{u})-a(\mathbf{v})\|_{X} \lesssim\|\mathbf{u}-\mathbf{v}\|
$$

for all $\mathbf{u}, \mathbf{v} \in \mathcal{B}_{\delta / c}$.

Proof. First, let $c>0, \delta>0$ be such that Lemma 6.3 holds. For fixed $\mathbf{u} \in \mathcal{B}_{\delta / c}$ there is a unique $a=a_{\mathbf{u}}(\Phi) \in X_{\delta}$ associated to every $\Phi \in \mathcal{X}_{\delta}$, such that Eq. (6.7) and hence Eq. (6.6) are satisfied. We define $\mathbf{K}_{\mathbf{u}}(\Phi):=\mathbf{K}(\Phi, a, \mathbf{u})$ and show that $\mathbf{K}_{\mathbf{u}}$ maps $\mathcal{X}_{\delta}$ into itself. By transversality of the projections we have

$$
\begin{array}{r}
\mathbf{P}_{a_{\infty}} \mathbf{K}_{\mathbf{u}}(\Phi)(\tau)=-\int_{\tau}^{\infty} e^{\tau-\sigma} \mathbf{P}_{a_{\infty}}\left[\mathbf{G}_{a(\sigma)}(\Phi(\sigma))-\partial_{\sigma} \Psi_{a(\sigma)}^{*}\right] d \sigma \\
\mathbf{H}_{a_{\infty}} \mathbf{K}_{\mathbf{u}}(\Phi)(\tau)=-\int_{\tau}^{\infty} e^{3(\tau-\sigma)} \mathbf{H}_{a_{\infty}}\left[\mathbf{G}_{a(\sigma)}(\Phi(\sigma))-\partial_{\sigma} \Psi_{a(\sigma)}^{*}\right] d \sigma .
\end{array}
$$

To estimate these expressions we write $\partial_{\tau} \Psi_{a(\tau)}^{*}=\dot{a}_{j}(\tau) \mathbf{q}_{a_{\infty}}^{(j)}+\dot{a}_{j}(\tau)\left[\mathbf{q}_{a(\tau)}^{(j)}-\mathbf{q}_{a_{\infty}}^{(j)}\right]$ and use $\mathbf{H}_{a_{\infty}} \mathbf{q}_{a_{\infty}}^{(j)}=\mathbf{P}_{a_{\infty}}^{(k)} \mathbf{q}_{a_{\infty}}^{(j)}=0$ for $k=0, \ldots, 7$ together with Eq. (6.10) to infer that

$$
\left\|\mathbf{H}_{a_{\infty}} \partial_{\tau} \Psi_{a(\tau)}^{*}\right\|+\left\|\mathbf{P}_{a_{\infty}}^{(k)} \partial_{\tau} \Psi_{a(\tau)}^{*}\right\| \lesssim \delta^{2} e^{-2 \omega \tau}
$$

for all $a \in X_{\delta}$. Note that by the same reasoning, we get

$$
\left\|\left(1-\mathbf{Q}_{a_{\infty}}\right) \partial_{\tau} \Psi_{a(\tau)}^{*}\right\| \lesssim \delta^{2} e^{-2 \omega \tau} .
$$

With Lemma 6.2 we obtain

$$
\left\|\mathbf{H}_{a_{\infty}} \mathbf{K}_{\mathbf{u}}(\Phi)(\tau)\right\|+\left\|\mathbf{P}_{\infty} \mathbf{K}_{\mathbf{u}}(\Phi)(\tau)\right\| \lesssim \delta^{2} e^{-2 \omega \tau} .
$$

By Eq. (6.6), $\mathbf{Q}_{a_{\infty}} \mathbf{K}_{\mathbf{u}}(\Phi)(\tau)=\chi(\tau) \mathbf{Q}_{a_{\infty}} \mathbf{u}$ which implies that

$$
\left\|\mathbf{Q}_{a_{\infty}} \mathbf{K}_{\mathbf{u}}(\Phi)(\tau)\right\| \lesssim \frac{\delta}{c} e^{-2 \omega \tau}
$$

On the stable subspace we have

$$
\begin{aligned}
\mathbf{T}_{a_{\infty}} \mathbf{K}_{\mathbf{u}}(\Phi)(\tau) & =\left[1-\mathbf{H}_{a_{\infty}}-\mathbf{P}_{a_{\infty}}-\mathbf{Q}_{a_{\infty}}\right] \mathbf{K}_{\mathbf{u}}(\Phi)(\tau) \\
& =\mathbf{S}_{a_{\infty}}(\tau) \mathbf{T}_{a_{\infty}} \mathbf{u}+\int_{0}^{\tau} \mathbf{S}_{a_{\infty}}(\tau-\sigma) \mathbf{T}_{a_{\infty}}\left[\mathbf{G}_{a(\sigma)}(\Phi(\sigma))-\partial_{\sigma} \Psi_{a(\sigma)}^{*}\right] d \sigma .
\end{aligned}
$$

With Lemma 6.2 and Theorem 5.8,

$$
\left\|\mathbf{T}_{a_{\infty}} \mathbf{K}_{\mathbf{u}}(\Phi)(\tau)\right\| \lesssim\left(\frac{\delta}{c}+\delta^{2}\right) e^{-\omega \tau}
$$

These bounds imply that $\mathbf{K}_{\mathbf{u}}: \mathcal{X}_{\delta} \rightarrow \mathcal{X}_{\delta}$ for $\delta>0$ sufficiently small and $c>0$ sufficiently large. We claim that

$$
\left\|\mathbf{K}_{\mathbf{u}}(\Phi)-\mathbf{K}_{\mathbf{u}}(\Psi)\right\|_{\mathcal{X}} \lesssim \delta\|\Phi-\Psi\|_{\mathcal{X}}
$$

for all $\Phi, \Psi \in \mathcal{X}_{\delta}$ and $\delta$ sufficiently small. For this, we use that for all $a, b \in X_{\delta}$ with $\delta$ sufficiently small,

$$
\left\|\mathbf{P}_{a_{\infty}}^{(k)} \partial_{\tau} \Psi_{a(\tau)}^{*}-\mathbf{P}_{b_{\infty}}^{(k)} \partial_{\tau} \Psi_{b(\tau)}^{*}\right\|+\left\|\mathbf{H}_{a_{\infty}} \partial_{\tau} \Psi_{a(\tau)}^{*}-\mathbf{H}_{b_{\infty}} \partial_{\tau} \Psi_{b(\tau)}^{*}\right\| \lesssim \delta e^{-2 \omega \tau}\|a-b\|_{X},
$$

for $k=0, \ldots, 7$ and

$$
\left\|\left(1-\mathbf{Q}_{a_{\infty}}\right) \partial_{\tau} \Psi_{a(\tau)}^{*}-\left(1-\mathbf{Q}_{b_{\infty}}\right) \partial_{\tau} \Psi_{b(\tau)}^{*}\right\| \lesssim \delta e^{-2 \omega \tau}\|a-b\|_{X}
$$


These estimates are proved similarly to Eqns. (6.13)-(6.14) by using Eq. (6.11) and Lemma 5.7 in addition. Now, let $a=a_{\mathbf{u}}(\Phi), b=b_{\mathbf{u}}(\Psi) \in X_{\delta}$ be assigned to $\Phi, \Psi \in \mathcal{X}_{\delta}$ via Lemma 6.3. With Eq. (6.16), Lemma 6.2, Lemma 5.7,

$$
\begin{aligned}
\| \mathbf{P}_{a_{\infty}} \mathbf{K}_{\mathbf{u}}(\Phi)(\tau) & -\mathbf{P}_{b_{\infty}} \mathbf{K}_{\mathbf{u}}(\Psi)(\tau)\|+\| \mathbf{H}_{a_{\infty}} \mathbf{K}_{\mathbf{u}}(\Phi)(\tau)-\mathbf{H}_{b_{\infty}} \mathbf{K}_{\mathbf{u}}(\Psi)(\tau) \| \\
& \lesssim \delta e^{-2 \omega \tau}\left(\|\Phi-\Psi\|_{\mathcal{X}}-\|a-b\|_{X}\right) \lesssim \delta e^{-2 \omega \tau}\|\Phi-\Psi\|_{\mathcal{X}}
\end{aligned}
$$

where we have used the Lipschitz bounds of Lemma 6.3 in the last step. Eq. (6.6) implies that

$$
\left\|\mathbf{Q}_{a_{\infty}} \mathbf{K}_{\mathbf{u}}(\Phi)(\tau)-\mathbf{Q}_{b_{\infty}} \mathbf{K}_{\mathbf{u}}(\Psi)(\tau)\right\| \lesssim \delta e^{-2 \omega \tau}\|a-b\|_{X} \lesssim \delta e^{-2 \omega \tau}\|\Phi-\Psi\|_{\mathcal{X}}
$$

Finally, it is easy to check that

$$
\left\|\mathbf{T}_{a_{\infty}} \mathbf{K}_{\mathbf{u}}(\Phi)(\tau)-\mathbf{T}_{b_{\infty}} \mathbf{K}_{\mathbf{u}}(\Psi)(\tau)\right\| \lesssim \delta e^{-\omega \tau}\|\Phi-\Psi\|_{\mathcal{X}}
$$

By combining these estimates we infer that Eq. (6.15) holds and the existence of a solution follows from the contraction mapping principle, which applies provided $\delta>0$ is chosen sufficiently small.

Finally, we verify the Lipschitz continuity of the solution map. For $\mathbf{u}, \mathbf{v} \in \mathcal{B}_{\delta / c}$, there are unique $(\Phi, a),(\Psi, b) \in \mathcal{X}_{\delta} \times X_{\delta}$ such that $\mathbf{Q}_{a_{\infty}} \Phi=\chi \mathbf{Q}_{a_{\infty}} \mathbf{u}, \mathbf{Q}_{b_{\infty}} \Psi=\chi \mathbf{Q}_{b_{\infty}} \mathbf{v}$ and $\Phi=$ $\mathbf{K}(\Phi, a, \mathbf{u})$ as well as $\Psi=\mathbf{K}(\Psi, b, \mathbf{v})$. We show that

$$
\|\Phi-\Psi\|_{\mathcal{X}} \lesssim\|\mathbf{u}-\mathbf{v}\|
$$

for all $\mathbf{u}, \mathbf{v} \in \mathcal{B}_{\delta / c}$. First, we have

$$
\begin{aligned}
\| \mathbf{Q}_{a_{\infty}} \mathbf{K}(\Phi, a, \mathbf{u})(\tau) & -\mathbf{Q}_{b_{\infty}} \mathbf{K}(\Psi, b, \mathbf{v})(\tau)\|\lesssim\| \chi(\tau)\left[\mathbf{Q}_{a_{\infty}} \mathbf{u}-\mathbf{Q}_{b_{\infty}} \mathbf{v}\right] \| \\
& \lesssim e^{-\omega \tau}\left(\delta\|a-b\|_{X}+\|\mathbf{u}-\mathbf{v}\|\right) \lesssim e^{-\omega \tau}\left(\delta\|\Phi-\Psi\|_{\mathcal{X}}+\|\mathbf{u}-\mathbf{v}\|\right)
\end{aligned}
$$

by Lemma 6.3. The expressions $\mathbf{P}_{a_{\infty}} \mathbf{K}(\Phi, a, \mathbf{u})$ and $\mathbf{H}_{a_{\infty}} \mathbf{K}(\Phi, a, \mathbf{u})$ do not depend on $\mathbf{u}$ explicitly and analogously to Eq. (6.17) we obtain

$$
\begin{aligned}
\| \mathbf{P}_{a_{\infty}} \mathbf{K}(\Phi, a, \mathbf{u})(\tau) & -\mathbf{P}_{b_{\infty}} \mathbf{K}(\Psi, b, \mathbf{v})(\tau) \| \\
& +\left\|\mathbf{H}_{a_{\infty}} \mathbf{K}(\Phi, a, \mathbf{u})(\tau)-\mathbf{H}_{b_{\infty}} \mathbf{K}(\Psi, b, \mathbf{v})(\tau)\right\| \lesssim \delta e^{-2 \omega \tau}\|\Phi-\Psi\|_{\mathcal{X}} .
\end{aligned}
$$

Finally,

$$
\begin{aligned}
\| \mathbf{S}_{a_{\infty}}(\tau) \mathbf{T}_{a_{\infty}} \mathbf{u} & -\mathbf{S}_{b_{\infty}}(\tau) \mathbf{T}_{b_{\infty}} \mathbf{v}\left\|\lesssim e^{-\omega \tau}\right\| a-b\left\|_{X}\right\| \mathbf{u}\left\|+e^{-\omega \tau}\right\| \mathbf{u}-\mathbf{v} \| \\
& \lesssim e^{-\omega \tau}\left(\delta\|\Phi-\Psi\|_{\mathcal{X}}+\|\mathbf{u}-\mathbf{v}\|\right)
\end{aligned}
$$

by Theorem 5.8. With this, the bound

$$
\left\|\mathbf{T}_{a_{\infty}} \mathbf{K}(\Phi, a, \mathbf{u})(\tau)-\mathbf{T}_{b_{\infty}} \mathbf{K}(\Psi, b, \mathbf{v})(\tau)\right\| \lesssim e^{-\omega \tau}\left(\delta\|\Phi-\Psi\|_{\mathcal{X}}+\|\mathbf{u}-\mathbf{v}\|\right)
$$

can be proved analogous to Eq. (6.18). A combination of these estimates shows that

$$
\|\Phi-\Psi\|_{\mathcal{X}}=\|\mathbf{K}(\Phi, a, \mathbf{u})-\mathbf{K}(\Psi, b, \mathbf{v})\|_{\mathcal{X}} \lesssim \delta\|\Phi-\Psi\|_{\mathcal{X}}+\|\mathbf{u}-\mathbf{v}\|
$$

and Eq. (6.19) follows for $\delta>0$ chosen sufficiently small. Hence, by Lemma 6.3,

$$
\|a-b\|_{X} \lesssim\|\Phi-\Psi\|_{\mathcal{X}}+\|\mathbf{u}-\mathbf{v}\| \lesssim\|\mathbf{u}-\mathbf{v}\|
$$

which implies the claim. 
6.3. Variation of blowup parameters - Co-dimension one stability. In this last section, we consider Eq. (6.12) with $\mathbf{u}=\mathbf{U}\left((f, g), T, x_{0}\right)$. We show that all instabilities, except from for the genuine one, can be eliminated by a suitable choice of the blowup parameters $\left(T, x_{0}\right)$.

6.3.1. Initial data operator. In the following, we properly define the initial data operator $\mathbf{U}$, see Eq. (2.8), which can be written as

$$
\mathbf{U}\left(\mathbf{v}, T, x_{0}\right)=\mathcal{R}\left(\mathbf{v}, T, x_{0}\right)+\mathcal{R}\left(\Psi_{0}^{*}, T, x_{0}\right)-\mathcal{R}\left(\Psi_{0}^{*}, 1,0\right),
$$

for $\mathbf{v}=\left(v_{1}, v_{2}\right)$. Since we expect $\left(T, x_{0}\right)$ to be close to $(1,0)$ we a priori assume that $x_{0} \in \mathbb{B}_{1 / 2}^{7}$ and that $T \in I:=\left[\frac{1}{2}, \frac{3}{2}\right]$. Furthermore, we define the Hilbert space

$$
\mathcal{Y}:=H^{4} \times H^{3}\left(\mathbb{B}_{2}^{7}\right)
$$

and denote by $\mathcal{B}_{\mathcal{Y}}$ the unit ball in $\mathcal{Y}$.

Lemma 6.5. Let $\delta>0$ be sufficiently small. The initial data operator $\mathbf{U}: \mathcal{B}_{\mathcal{Y}} \times I \times \mathbb{B}_{1 / 2}^{7} \rightarrow \mathcal{H}$ is Lipschtiz continuous, i.e.,

$$
\left\|\mathbf{U}\left(\mathbf{v}, T_{1}, x_{0}\right)-\mathbf{U}\left(\mathbf{w}, T_{2}, y_{0}\right)\right\| \lesssim\|\mathbf{v}-\mathbf{w}\|_{\mathcal{Y}}+\left|T_{1}-T_{2}\right|-\left|x_{0}-y_{0}\right|
$$

for all $\mathbf{v}, \mathbf{w} \in \mathcal{B}_{\mathcal{Y}}$, all $T_{1}, T_{2} \in I$ and all $x_{0}, y_{0} \in \mathbb{B}_{1 / 2}^{7}$. Furthermore, if $\|\mathbf{v}\|_{\mathcal{Y}} \leq \delta$, then for all $T \in[1-\delta, 1+\delta] \subset I$ and all $x_{0} \in \mathbb{B}_{\delta}^{7}$,

$$
\left\|\mathbf{U}\left(\mathbf{v}, T, x_{0}\right)\right\| \lesssim \delta
$$

Proof. Let $v \in C^{\infty}\left(\mathbb{B}_{2}^{7}\right)$. For all $T \in I$ and all $x_{0}, y_{0} \in \mathbb{B}_{1 / 2}^{7}$, we have

$$
v\left(T \xi+x_{0}\right)-v\left(T \xi+y_{0}\right)=\left(x_{0}^{j}-y_{0}^{j}\right) \int_{0}^{1} \partial_{j} v\left(T \xi+y_{0}+s\left(x_{0}-y_{0}\right)\right) d s,
$$

which implies that $\left\|v\left(T \cdot+x_{0}\right)-v\left(T \cdot+y_{0}\right)\right\|_{L^{2}\left(\mathbb{B}^{7}\right)} \lesssim\|v\|_{H^{1}\left(\mathbb{B}_{2}^{7}\right)}\left|x_{0}-y_{0}\right|$. The same argument shows that for fixed $k \in \mathbb{N}$,

$$
\left\|v\left(T \cdot+x_{0}\right)-v\left(T \cdot+y_{0}\right)\right\|_{H^{k}\left(\mathbb{B}^{7}\right)} \lesssim\|v\|_{H^{k+1}\left(\mathbb{B}_{2}^{7}\right)}\left|x_{0}-y_{0}\right| .
$$

Similarly, for all $T_{1}, T_{2} \in I$ and all $x_{0} \in \mathbb{B}_{1 / 2}^{7}$

$$
v\left(T_{1} \xi+x_{0}\right)-v\left(T_{2} \xi+x_{0}\right)=\left(T_{1}-T_{2}\right) \int_{0}^{1} \xi^{j} \partial_{j} v\left(\left(T_{2}+s\left(T_{1}-T_{2}\right)\right) \xi+x_{0}\right) d s,
$$

and thus

$$
\left\|v\left(T_{1} \cdot+x_{0}\right)-v\left(T_{2} \cdot+x_{0}\right)\right\|_{L^{2}\left(\mathbb{B}^{7}\right)} \lesssim\|(\cdot) \nabla v\|_{L^{2}\left(\mathbb{B}_{2}^{7}\right)}\left|T_{1}-T_{2}\right| \lesssim\|v\|_{H^{1}\left(\mathbb{B}_{2}^{7}\right)}\left|T_{1}-T_{2}\right|,
$$

where we have used that $\left|\xi^{j} \partial_{j} v(\xi)\right|^{2} \leq|\xi|^{2} \partial_{j} v(\xi) \overline{\partial^{j} v(\xi)}$. Analogously, for fixed $k \in \mathbb{N}$, we have

$$
\left\|v\left(T_{1} \cdot+x_{0}\right)-v\left(T_{2} \cdot+x_{0}\right)\right\|_{H^{k}\left(\mathbb{B}^{7}\right)} \lesssim\left|T_{1}-T_{2}\right|\|v\|_{H^{k+1}\left(\mathbb{B}_{2}^{7}\right)} .
$$

By a density argument Eqns. (6.20) - (6.21) can be extended to all $v \in H^{k+1}\left(\mathbb{B}_{2}^{7}\right)$. Now let $\mathbf{v} \in \mathcal{Y}$. Then, for all $T_{1}, T_{2} \in I$, all $x_{0}, y_{0} \in \mathbb{B}_{1 / 2}^{7}$, and all $\mathbf{v}, \mathbf{w} \in \mathcal{Y}$, Eqns. (6.20) - (6.21) imply that

$$
\left\|\mathcal{R}\left(\mathbf{v}, T_{1}, x_{0}\right)-\mathcal{R}\left(\mathbf{w}, T_{2}, y_{0}\right)\right\| \lesssim\|\mathbf{v}\|_{\mathcal{Y}}\left(\left|T_{1}-T_{2}\right|+\left|x_{0}-y_{0}\right|\right)+\|\mathbf{v}-\mathbf{w}\|_{\mathcal{Y}} .
$$


Since $\Psi_{0}^{*}$ is defined and smooth on all of $\mathbb{R}^{7}$ we immediately obtain

$$
\left\|\mathcal{R}\left(\Psi_{0}^{*}, T_{1}, x_{0}\right)-\mathcal{R}\left(\Psi_{0}^{*}, T_{2}, y_{0}\right)\right\| \lesssim\left|T_{1}-T_{2}\right|+\left|x_{0}-y_{0}\right|
$$

for all $T_{1}, T_{2} \in I$ and all $x_{0}, y_{0} \in \mathbb{B}_{1 / 2}^{7}$. Finally, the bound

$$
\left\|\mathbf{U}\left(\mathbf{v}, T, x_{0}\right) \lesssim\right\| \mathbf{v} \|_{\mathcal{Y}}+|T-1|+\left|x_{0}\right|
$$

implies the claimed smallness of $\mathbf{U}$.

This yields the following corollary to Proposition 6.4.

Corollary 6.6. Let $M>0$ be sufficiently large and $\delta>0$ be sufficiently small. Then, for every $\|\mathbf{v}\|_{\mathcal{Y}} \leq \frac{\delta}{M}$, every $T \in\left[1-\frac{\delta}{M}, 1+\frac{\delta}{M}\right] \subset I$ and every $x_{0} \in \overline{\mathbb{B}_{\delta / M}^{7}}$ there exist functions $\Phi \in \mathcal{X}_{\delta}$ and $a \in X_{\delta}$ such that the integral equation

$\Phi(\tau)=\mathbf{S}_{a_{\infty}}(\tau)\left[\mathbf{U}\left(\mathbf{v}, T, x_{0}\right)-\mathbf{C}\left(\Phi, a, \mathbf{U}\left(\mathbf{v}, T, x_{0}\right)\right)\right]+\int_{0}^{\tau} \mathbf{S}_{a_{\infty}}(\tau-\sigma)\left[\mathbf{G}_{a(\sigma)}(\Phi(\sigma))-\partial_{\sigma} \Psi_{a(\sigma)}^{*}\right] d \sigma$ is satisfied for all $\tau \geq 0$. In particular, $\Phi$ decays exponentially,

$$
\|\Phi(\tau)\| \lesssim \delta e^{-\omega \tau}, \quad \forall \tau \geq 0
$$

Furthermore, the solution map $\left(\mathbf{v}, T, x_{0}\right) \mapsto(\Phi, a)$ satisfies

$$
\begin{aligned}
\left\|\Phi\left(\mathbf{v}, T, x_{0}\right)-\Phi\left(\mathbf{w}, \tilde{T}, y_{0}\right)\right\|_{\mathcal{X}} & +\left\|a\left(\mathbf{v}, T, x_{0}\right)-a\left(\mathbf{w}, \tilde{T}, y_{0}\right)\right\|_{X} \\
& \lesssim\|\mathbf{v}-\mathbf{w}\|_{\mathcal{Y}}+|T-\tilde{T}|+\left|x_{0}-\tilde{x}_{0}\right|
\end{aligned}
$$

for all $\mathbf{v}, \mathbf{w} \in \mathcal{Y}$ satisfying the smallness condition, all $T, \tilde{T} \in\left[1-\frac{\delta}{M}, 1+\frac{\delta}{M}\right]$ and all $x_{0}, \tilde{x}_{0} \in \overline{\mathbb{B}_{1 / 2}^{7}}$

6.3.2. Variation of blowup parameters. To shorten the notation, we set $\mathbf{h}:=\mathbf{h}_{0}$, where $\mathbf{h}_{0}$ is eigenfunction of $\mathbf{L}_{0}$ corresponding to the eigenvalue $\lambda=3$, see Proposition 5.3.

Proposition 6.7. Chose $c>0$ sufficiently large and $\delta>0$ sufficiently small. For every $\mathbf{v} \in \mathcal{Y}$ satisfying $\|\mathbf{v}\|_{\mathcal{Y}} \leq \frac{\delta}{c^{2}}$, there exist functions $\Phi \in \mathcal{X}_{\delta}, a \in X_{\delta}$ and parameters $\alpha \in\left[-\frac{\delta}{c}, \frac{\delta}{c}\right]$, $T \in I_{\delta / c}=\left[1-\frac{\delta}{c}, 1+\frac{\delta}{c}\right] \subset I, x_{0} \in \overline{\mathbb{B}_{\delta / c}^{7}} \subset \mathbb{B}_{1 / 2}^{7}$ such that

$$
\mathbf{C}\left(\Phi, a, \mathbf{U}\left(\mathbf{v}+\alpha \mathbf{h}, T, x_{0}\right)\right)=0 .
$$

Moreover, the parameters depend Lipschitz continuously on the data, i.e.,

$$
|\alpha(\mathbf{v})-\alpha(\mathbf{w})|+|T(\mathbf{v})-T(\mathbf{w})|+\left|x_{0}(\mathbf{v})-x_{0}(\mathbf{w})\right| \lesssim\|\mathbf{v}-\mathbf{w}\|_{\mathcal{Y}}
$$

for all $\mathbf{v}, \mathbf{w} \in \mathcal{Y}$ satisfying the above smallness assumption.

Proof. First, we observe that $\|\mathbf{v}+\alpha \mathbf{h}\| \leq \frac{\delta}{M}$ for $c>M$ sufficiently large, where $M>0$ is the constant in Corollary 6.6. Consequently, for fixed $\mathbf{v}$ and all $\alpha \in\left[-\frac{\delta}{c}, \frac{\delta}{c}\right], T \in I_{\delta / c}$ and $x_{0} \in$ $\overline{\mathbb{B}_{\delta / c}^{7}}$ there are functions $(\Phi, a) \in \mathcal{X}_{\delta} \times X_{\delta}, \Phi=\Phi\left(\mathbf{v}+\alpha \mathbf{h}, T, x_{0}\right), a=a\left(\mathbf{v}+\alpha \mathbf{h}, T, x_{0}\right)$ associated via Corollary 6.6. Note that the correction terms can be written as $\mathbf{C}_{1}=\sum_{k=0}^{7} \mathbf{C}_{1}^{(k)}$ with

$$
\mathbf{C}_{1}^{(k)}(\Phi, a, \mathbf{u})=\mathbf{P}_{a_{\infty}}^{(k)} \mathbf{u}+\mathbf{P}_{a_{\infty}}^{(k)} \mathbf{I}_{1}(\Phi, a)
$$

and

$$
\mathbf{C}_{2}(\Phi, a, \mathbf{u})=\underset{37}{\mathbf{H}_{a_{\infty}} \mathbf{u}}+\mathbf{H}_{a_{\infty}} \mathbf{I}_{2}(\Phi, a)
$$


where

$$
\begin{aligned}
& \mathbf{I}_{1}(\Phi, a):=\int_{0}^{\infty} e^{-\sigma}\left[\mathbf{G}_{a(\sigma)}(\Phi(\sigma))-\partial_{\sigma} \Psi_{a(\sigma)}^{*}\right] d \sigma, \\
& \mathbf{I}_{2}(\Phi, a):=\int_{0}^{\infty} e^{-3 \sigma}\left[\mathbf{G}_{a(\sigma)}(\Phi(\sigma))-\partial_{\sigma} \Psi_{a(\sigma)}^{*}\right] d \sigma .
\end{aligned}
$$

In view of Lemma 6.2 and Eq. (6.13) the integral terms can be estimated by

$$
\left\|\mathbf{P}_{a_{\infty}}^{(k)} \mathbf{I}_{1}(\Phi, a)\right\| \lesssim \delta^{2}, \quad\left\|\mathbf{H}_{a_{\infty}} \mathbf{I}_{2}(\Phi, a)\right\| \lesssim \delta^{2}
$$

We show that $\left(\alpha, T, x_{0}\right)$ can be chosen such that for $k=0, \ldots, 7$.

$$
\begin{aligned}
\left(\mathbf{C}_{1}^{(k)}\left(\Phi, a, \mathbf{U}\left(\mathbf{v}+\alpha \mathbf{h}, T, x_{0}\right)\right) \mid \mathbf{g}_{a_{\infty}}^{(k)}\right) & =0 \\
\left(\mathbf{C}_{2}\left(\Phi, a, \mathbf{U}\left(\mathbf{v}+\alpha \mathbf{h}, T, x_{0}\right)\right) \mid \mathbf{h}_{a_{\infty}}\right) & =0
\end{aligned}
$$

which implies Eq. (6.24). For this, we use the fact that

$$
\left.\partial_{T}\left[T \psi_{0,1}^{*}\left(T \xi+x_{0}\right)\right]\right|_{\left(T, x_{0}\right)=(1,0)}=c_{0} g_{0,1}(\xi),\left.\quad \partial_{x_{0}^{j}}\left[T \psi_{0,1}^{*}\left(T \xi+x_{0}\right)\right]\right|_{\left(T, x_{0}\right)=(1,0)}=c_{j} g_{0,1}^{(j)}(\xi)
$$

for $j=1, \ldots, 7$ and some constants $c_{0}, c_{j} \in \mathbb{R}$. The analogous statement holds for the second component $\psi_{0,2}^{*}$. By Taylor expansion we get that for all $T \in I_{\delta / c}$ and $x_{0} \in \overline{\mathbb{B}_{\delta / c}^{7}}$,

$$
\mathcal{R}\left(\Psi_{0}^{*}, T, x_{0}\right)-\mathcal{R}\left(\Psi_{0}^{*}, 1,0\right)=c_{0}(T-1) \mathbf{g}_{0}^{(0)}+\sum_{j=1}^{7} c_{j} x_{0}^{j} \mathbf{g}_{0}^{(j)}+R\left(T, x_{0}\right) .
$$

For all $T, \tilde{T} \in I_{\delta / c}$, and all $x_{0}, \tilde{x}_{0} \in \overline{\mathbb{B}_{\delta / c}^{7}}$, we have

$$
\left\|R\left(T, x_{0}\right)-R\left(\tilde{T}, \tilde{x}_{0}\right)\right\| \lesssim \delta\left(|T-\tilde{T}|+\left|x_{0}-\tilde{x}_{0}\right|\right)
$$

In the following, we write

$$
\begin{aligned}
\mathbf{U}\left(\mathbf{v}+\alpha \mathbf{h}, T, x_{0}\right) & =\mathcal{R}\left(\mathbf{v}+\alpha \mathbf{h}, T, x_{0}\right)+c_{0}(T-1) \mathbf{g}_{a_{\infty}}^{(0)}+\sum_{j=1}^{7} c_{j} x_{0}^{j} \mathbf{g}_{a_{\infty}}^{(j)} \\
& +c_{0}(T-1)\left[\mathbf{g}_{0}^{(0)}-\mathbf{g}_{a_{\infty}}^{(0)}\right]+\sum_{j=1}^{7} c_{j} x_{0}^{j}\left[\mathbf{g}_{0}^{(j)}-\mathbf{g}_{a_{\infty}}^{(j)}\right]+R\left(T, x_{0}\right) \\
& =\mathcal{R}\left(\mathbf{v}+\alpha \mathbf{h}, T, x_{0}\right)+c_{0}(T-1) \mathbf{g}_{a_{\infty}}^{(0)}+\sum_{j=1}^{7} c_{j} x_{0}^{j} \mathbf{g}_{a_{\infty}}^{(j)}+R_{a_{\infty}}\left(T, x_{0}\right),
\end{aligned}
$$

with

$$
R_{a_{\infty}}\left(T, x_{0}\right)=c_{0}(T-1)\left[\mathbf{g}_{0}^{(0)}-\mathbf{g}_{a_{\infty}}^{(0)}\right]+\sum_{j=1}^{7} c_{j} x_{0}^{j}\left[\mathbf{g}_{0}^{(j)}-\mathbf{g}_{a_{\infty}}^{(j)}\right]+R\left(T, x_{0}\right) .
$$

It is easy to check that the remainder term satisfies

$$
\left\|R_{a}\left(T, x_{0}\right)-R_{b}\left(\tilde{T}, \tilde{x}_{0}\right)\right\| \lesssim \delta\left(|a-b|+|T-\tilde{T}|+\left|x_{0}-\tilde{x}_{0}\right|\right)
$$

for all $a, b \in \mathbb{B}^{7}$ with $|a| \lesssim \delta,|b| \lesssim \delta$, all $T, \tilde{T} \in I_{\delta / c}$, and all $x_{0}, \tilde{x}_{0} \in \overline{\mathbb{B}_{\delta / c}^{7}}$. To further simplify the above expression, we write

$$
\mathcal{R}\left(\mathbf{v}+\alpha \mathbf{h}, T, x_{0}\right)=\mathcal{R}\left(\mathbf{v}, T, x_{0}\right)+\underset{38}{\alpha \mathcal{R}}\left(\mathbf{h}_{a_{\infty}}, T, x_{0}\right)+\alpha \mathcal{R}\left(\mathbf{h}-\mathbf{h}_{a_{\infty}}, T, x_{0}\right)
$$


The last term can be estimated by

$$
\left\|\mathcal{R}\left(\mathbf{h}-\mathbf{h}_{a_{\infty}}, T, x_{0}\right)\right\| \lesssim\left\|\mathbf{h}-\mathbf{h}_{a_{\infty}}\right\|_{H^{3} \times H^{2}\left(\mathbb{B}_{2}^{7}\right)} \lesssim\left|a_{\infty}\right|
$$

where the Lipschitz bound for $\mathbf{h}_{a}$ follows from its smoothness on $\overline{\mathbb{B}_{2}^{7}}$ for small $a$. By Taylor expansion of $\mathcal{R}\left(\mathbf{h}_{a_{\infty}}, T, x_{0}\right)$ at $\left(T, x_{0}\right)=(1,0)$ we obtain

$$
\mathcal{R}\left(\mathbf{v}+\alpha \mathbf{h}, T, x_{0}\right)=\mathcal{R}\left(\mathbf{v}, T, x_{0}\right)+\alpha \mathbf{h}_{a_{\infty}}+\alpha \tilde{R}_{a_{\infty}}\left(T, x_{0}\right)
$$

where

$$
\left\|\tilde{R}_{a}\left(T, x_{0}\right)-\tilde{R}_{b}\left(\tilde{T}, \tilde{x}_{0}\right)\right\| \lesssim|T-\tilde{T}|+\left|x_{0}-\tilde{x}_{0}\right|+|a-b| .
$$

In summary, we have

$$
\begin{aligned}
\mathbf{U}\left(\mathbf{v}+\alpha \mathbf{h}, T, x_{0}\right)=\mathcal{R}\left(\mathbf{v}, T, x_{0}\right) & +\alpha \mathbf{h}_{a_{\infty}}+c_{0}(T-1) \mathbf{g}_{a_{\infty}}^{(0)}+\sum_{j=1}^{7} c_{j} x_{0}^{j} \mathbf{g}_{a_{\infty}}^{(j)} \\
& +R_{a_{\infty}}\left(T, x_{0}\right)+\alpha \tilde{R}_{a_{\infty}}\left(T, x_{0}\right) .
\end{aligned}
$$

By transversality of the projections, we infer that for $j=1, \ldots, 7$,

$$
\begin{aligned}
\mathbf{P}_{a_{\infty}}^{(0)} \mathbf{U}\left(\mathbf{v}+\alpha \mathbf{h}, T, x_{0}\right) & =\mathbf{P}_{a_{\infty}}^{(0)} \mathcal{R}\left(\mathbf{v}, T, x_{0}\right)+c_{0}(T-1) \mathbf{g}_{a_{\infty}}^{(0)}+\mathbf{P}_{a_{\infty}}^{(0)} R_{a_{\infty}}\left(T, x_{0}\right)+\alpha \mathbf{P}_{a_{\infty}}^{(0)} \tilde{R}_{a_{\infty}}\left(T, x_{0}\right), \\
\mathbf{P}_{a_{\infty}}^{(j)} \mathbf{U}\left(\mathbf{v}+\alpha \mathbf{h}, T, x_{0}\right) & =\mathbf{P}_{a_{\infty}}^{(j)} \mathcal{R}\left(\mathbf{v}, T, x_{0}\right)+c_{j} x_{0}^{j} \mathbf{g}_{a_{\infty}}^{(j)}+\mathbf{P}_{a_{\infty}}^{(j)} R_{a_{\infty}}\left(T, x_{0}\right)+\alpha \mathbf{P}_{a_{\infty}}^{(j)} \tilde{R}_{a_{\infty}}\left(T, x_{0}\right), \\
\mathbf{H}_{a_{\infty}} \mathbf{U}\left(\mathbf{v}+\alpha \mathbf{h}, T, x_{0}\right) & =\mathbf{H}_{a_{\infty}} \mathcal{R}\left(\mathbf{v}, T, x_{0}\right)+\alpha \mathbf{h}_{a_{\infty}}+\mathbf{H}_{a_{\infty}} R_{a_{\infty}}\left(T, x_{0}\right)+\alpha \mathbf{H}_{a_{\infty}} \tilde{R}_{a_{\infty}}\left(T, x_{0}\right) .
\end{aligned}
$$

In the following, we write $T=1+\beta$ for $\beta \in \overline{\mathbb{B}_{\delta / c}}$ and define for $k=0, \ldots, 7$,

$\boldsymbol{\Gamma}_{\mathbf{v}}^{(k)}\left(\alpha, \beta, x_{0}\right):=\mathbf{P}_{a_{\infty}}^{(k)} \mathcal{R}\left(\mathbf{v}, 1+\beta, x_{0}\right)+\mathbf{P}_{a_{\infty}}^{(k)} R_{a_{\infty}}\left(\beta, x_{0}\right)+\alpha \mathbf{P}_{a_{\infty}}^{(k)} \tilde{R}_{a_{\infty}}\left(\beta, x_{0}\right)+\mathbf{P}_{a_{\infty}}^{(k)} \mathbf{I}_{1}\left(\alpha, \beta, x_{0}\right)$,

$\Gamma_{\mathbf{v}}^{(8)}\left(\alpha, \beta, x_{0}\right):=\mathbf{H}_{a_{\infty}} \mathcal{R}\left(\mathbf{v}, 1+\beta, x_{0}\right)+\mathbf{H}_{a_{\infty}} R_{a_{\infty}}\left(\beta, x_{0}\right)+\alpha \mathbf{H}_{a_{\infty}} \tilde{R}_{a_{\infty}}\left(\beta, x_{0}\right)+\mathbf{H}_{a_{\infty}} \mathbf{I}_{2}\left(\alpha, \beta, x_{0}\right)$

by slight abuse of notation. Then Eq. (6.26) can be written as

$$
\begin{gathered}
\beta=\Gamma_{\mathbf{v}}^{(0)}\left(\alpha, \beta, x_{0}\right)=\tilde{c}_{0}\left(\boldsymbol{\Gamma}_{\mathbf{v}}^{(0)}\left(\alpha, \beta, x_{0}\right) \mid \mathbf{g}_{a_{\infty}}^{(0)}\right) \\
x_{0}^{j}=\Gamma_{\mathbf{v}}^{(j)}\left(\alpha, \beta, x_{0}\right)=\tilde{c}_{j}\left(\boldsymbol{\Gamma}_{\mathbf{v}}^{(j)}\left(\alpha, \beta, x_{0}\right) \mid \mathbf{g}_{a_{\infty}}^{(j)}\right) \\
\alpha=\Gamma_{\mathbf{v}}^{(8)}\left(\alpha, \beta, x_{0}\right)=\tilde{c}_{8}\left(\boldsymbol{\Gamma}_{\mathbf{v}}^{(8)}\left(\alpha, \beta, x_{0}\right) \mid \mathbf{h}_{a_{\infty}}\right)
\end{gathered}
$$

for $j=1, \ldots, 7$ some constants $\tilde{c}_{0}, \tilde{c}_{j}, \tilde{c}_{8} \in \mathbb{R}$. First, we show that $\Gamma_{\mathbf{v}}=\left(\Gamma_{\mathbf{v}}^{(0)}, \ldots, \Gamma_{\mathbf{v}}^{(8)}\right)$ maps $\overline{\mathbb{B}_{\delta / c}^{9}}$ to itself for suitably large $c>0$ and suitably small $\delta>0$. Since $\left\|\mathcal{R}\left(\mathbf{v}, 1+\beta, x_{0}\right)\right\| \lesssim\|\mathbf{v}\|_{\mathcal{Y}}$, Eqns. (6.27) - (6.28) and Eq. (6.25) imply that

$$
\Gamma_{\mathbf{v}}^{(i)}\left(\alpha, \beta, x_{0}\right)=O\left(\frac{\delta}{c^{2}}\right)+O\left(\delta^{2}\right)
$$

for $i=0, \ldots, 8$. In particular, for $c>0$ sufficiently large and $\delta=\delta(c)$ sufficiently small, we obtain

$$
\left|\Gamma_{\mathbf{v}}\left(\alpha, \beta, x_{0}\right)\right| \leq \frac{\delta}{c}
$$

Next, we show that $\Gamma_{\mathbf{v}}: \overline{\mathbb{B}_{\delta / c}^{9}} \rightarrow \overline{\mathbb{B}_{\delta / c}^{9}}$ is contracting. Let again $(\Phi, a) \in \mathcal{X}_{\delta} \times X_{\delta}$ be the functions associated to $\mathbf{v}_{\alpha}=\mathbf{v}+\alpha \mathbf{h}, T=1+\beta$ and $x_{0}$ via Corollary 6.6. Furthermore, let 
$(\Psi, b) \in \mathcal{X}_{\delta} \times X_{\delta}$ be associated to $\tilde{\mathbf{v}}_{\alpha}=\mathbf{v}+\tilde{\alpha} \mathbf{h}, \tilde{T}=1+\tilde{\beta}$ and $\tilde{x}_{0}$. By Proposition 6.4 and Lemma 6.5, we have

$$
\begin{aligned}
\|\Phi-\Psi\|_{\mathcal{X}}+\|a-b\|_{X} & \lesssim\left\|\mathbf{U}\left(\mathbf{v}+\alpha \mathbf{h}, T, x_{0}\right)-\mathbf{U}\left(\mathbf{v}+\tilde{\alpha} \mathbf{h}, \tilde{T}, \tilde{x}_{0}\right)\right\| \\
& \lesssim|\alpha-\tilde{\alpha}|+|\beta-\tilde{\beta}|+\left|x_{0}-\tilde{x}_{0}\right| .
\end{aligned}
$$

Thus, by Lemma 6.2 and Eq. (6.16),

$\left\|\mathbf{P}_{a_{\infty}}^{(k)} \mathbf{I}_{1}(\Phi, a)-\mathbf{P}_{b_{\infty}}^{(k)} \mathbf{I}_{1}(\Psi, b)\right\| \lesssim \delta\left(\|a-b\|_{X}+\|\Phi-\Psi\|_{\mathcal{X}}\right) \lesssim \delta\left(|\alpha-\tilde{\alpha}|+|\beta-\tilde{\beta}|+\left|x_{0}-\tilde{x}_{0}\right|\right)$,

for $k=0, \ldots, 7$, and

$$
\left\|\mathbf{H}_{a_{\infty}} \mathbf{I}_{2}(\Phi, a)-\mathbf{H}_{b_{\infty}} \mathbf{I}_{2}(\Psi, b)\right\| \lesssim \delta\left(|\alpha-\tilde{\alpha}|+|\beta-\tilde{\beta}|+\left|x_{0}-\tilde{x}_{0}\right|\right) .
$$

Furthermore, using Eq. (6.22) and the Lipschitz continuity of the operators $\mathbf{P}_{a}^{(k)}, \mathbf{H}_{a}$ we get

$$
\begin{aligned}
& \left\|\mathbf{P}_{a_{\infty}}^{(k)} \mathcal{R}\left(\mathbf{v}, T, x_{0}\right)-\mathbf{P}_{b_{\infty}}^{(k)} \mathcal{R}\left(\mathbf{v}, \tilde{T}, \tilde{x}_{0}\right)\right\|+\left\|\mathbf{H}_{a_{\infty}} \mathcal{R}\left(\mathbf{v}, T, x_{0}\right)-\mathbf{H}_{b_{\infty}} \mathcal{R}\left(\mathbf{v}, \tilde{T}, \tilde{x}_{0}\right)\right\| \\
& \quad \lesssim\|\mathbf{v}\|_{\mathcal{Y}}\left(\|a-b\|_{X}+|T-\tilde{T}|+\left|x_{0}-\tilde{x}_{0}\right|\right) \lesssim \delta\left(|\alpha-\tilde{\alpha}|+|\beta-\tilde{\beta}|+\left|x_{0}-\tilde{x}_{0}\right|\right) .
\end{aligned}
$$

Furthermore,

$$
\begin{aligned}
\left\|\mathbf{P}_{a_{\infty}}^{(k)} R_{a_{\infty}}\left(T, x_{0}\right)-\mathbf{P}_{b_{\infty}}^{(k)} R_{b_{\infty}}\left(\tilde{T}, \tilde{x}_{0}\right)\right\| & +\left\|\alpha \mathbf{P}_{a_{\infty}}^{(k)} \tilde{R}_{a_{\infty}}\left(T, x_{0}\right)-\tilde{\alpha} \mathbf{P}_{b_{\infty}}^{(k)} \tilde{R}_{b_{\infty}}\left(\tilde{T}, \tilde{x}_{0}\right)\right\| \\
& \lesssim \delta\left(|\alpha-\tilde{\alpha}|+|\beta-\tilde{\beta}|+\left|x_{0}-\tilde{x}_{0}\right|\right)
\end{aligned}
$$

for $k=0, \ldots, 7$ and analogous estimates hold for $\mathbf{H}_{a_{\infty}}$. By combining these bounds we obtain that for $i=0, \ldots, 8$,

$$
\left|\Gamma_{\mathbf{v}}^{(i)}\left(\alpha, \beta, x_{0}\right)-\Gamma_{\mathbf{v}}^{(i)}\left(\tilde{\alpha}, \tilde{\beta}, \tilde{x}_{0}\right)\right| \lesssim \delta\left(|\alpha-\tilde{\alpha}|+|\beta-\tilde{\beta}|+\left|x_{0}-\tilde{x}_{0}\right|\right) .
$$

In particular, $\Gamma_{\mathbf{v}}$ is contracting for $\delta$ chosen sufficiently small. An application of the Banach fixed point theorem implies the first part of the statement.

It remains to show that the parameters depend Lipschitz continuously on $\mathbf{v}$. To keep track of the dependencies we denote by $\left(\Phi\left(\mathbf{v}_{\alpha}, \beta, x_{0}\right), a\left(\mathbf{v}_{\alpha}, \beta, x_{0}\right)\right)$ the functions $(\Phi, a)$ associated to $\mathbf{v}+\alpha \mathbf{h}, T=1+\beta, x_{0}$ via Corollary 6.6. For $\mathbf{v}, \mathbf{w} \in \mathcal{Y}$ satisfying the required smallness condition, let $\left(\alpha, \beta, x_{0}\right),\left(\tilde{\alpha}, \tilde{\beta}, \tilde{x}_{0}\right)$ be the corresponding unique set of parameters, such that Eq. (6.29) is satisfied. The first component of Eq. (6.29) implies that

$$
\begin{aligned}
|\beta-\tilde{\beta}| & =\left|\Gamma_{\mathbf{v}}^{(0)}\left(\alpha, \beta, x_{0}\right)-\Gamma_{\mathbf{w}}^{(0)}\left(\tilde{\alpha}, \tilde{\beta}, \tilde{x}_{0}\right)\right| \leq\left|\Gamma_{\mathbf{v}}^{(0)}\left(\alpha, \beta, x_{0}\right)-\Gamma_{\mathbf{w}}^{(0)}\left(\alpha, \beta, x_{0}\right)\right| \\
& +\left|\Gamma_{\mathbf{w}}^{(0)}\left(\alpha, \beta, x_{0}\right)-\Gamma_{\mathbf{w}}^{(0)}\left(\tilde{\alpha}, \tilde{\beta}, \tilde{x}_{0}\right)\right| .
\end{aligned}
$$

For the last term, we use Eq. (6.30). To estimate the first term, we use the Lipschitz continuity of the projections together with Corollary 6.6 to get

$$
\begin{aligned}
\| \mathbf{P}_{a_{\infty}\left(\mathbf{v}_{\alpha}, \beta, x_{0}\right)}^{(0)} & \mathcal{R}\left(\mathbf{v}, 1+\beta, x_{0}\right)-\mathbf{P}_{a_{\infty}\left(\mathbf{w}_{\alpha}, \beta, x_{0}\right)}^{(0)} \mathcal{R}\left(\mathbf{w}, 1+\beta, x_{0}\right) \| \\
& \lesssim\|\mathbf{v}\|_{\mathcal{Y}}\left\|a_{\infty}\left(\mathbf{v}_{\alpha}, \beta, x_{0}\right)-a_{\infty}\left(\mathbf{w}_{\alpha}, \beta, x_{0}\right)\right\|_{X}+\|\mathbf{v}-\mathbf{w}\|_{\mathcal{Y}} \lesssim\|\mathbf{v}-\mathbf{w}\|_{\mathcal{Y}} .
\end{aligned}
$$

Similar estimates exploiting Eqns. (6.27)-(6.28), Lemma 6.2 and Eq. (6.16) yield

$$
\left|\Gamma_{\mathbf{v}}^{(0)}\left(\alpha, \beta, x_{0}\right)-\Gamma_{\mathbf{w}}^{(0)}\left(\alpha, \beta, x_{0}\right)\right| \lesssim\|\mathbf{v}-\mathbf{w}\|_{\mathcal{Y}}
$$

In summary, we obtain

$$
|\beta-\tilde{\beta}| \lesssim \delta\left(|\alpha-\tilde{\alpha}|+|\beta-\tilde{\beta}|+\left|x_{0}-\tilde{x}_{0}\right|\right)+\|\mathbf{v}-\mathbf{w}\|_{\mathcal{Y}}
$$


Analogous estimates for the remaining components show that

$$
|\alpha-\tilde{\alpha}|+|\beta-\tilde{\beta}|+\left|x_{0}-\tilde{x}_{0}\right| \lesssim \delta\left(|\alpha-\tilde{\alpha}|+|\beta-\tilde{\beta}|+\left|x_{0}-\tilde{x}_{0}\right|\right)+\|\mathbf{v}-\mathbf{w}\|_{\mathcal{Y}},
$$

which implies the claim provided that $\delta$ is chosen sufficiently small.

\subsection{Proof of Theorem 1.1.}

Proof. Let $\delta>0$ and $c>0$ be such that Proposition 6.7 holds. Define $\delta^{\prime}:=\frac{\delta}{c}$. Let $\mathbf{v}=\left(v_{1}, v_{2}\right) \in C^{\infty}\left(\overline{\mathbb{B}_{2}^{7}}\right) \times C^{\infty}\left(\overline{\mathbb{B}_{2}^{7}}\right)$ satisfy

$$
\|\mathbf{v}\|_{H^{4} \times H^{3}\left(\mathbb{B}_{2}^{7}\right)} \leq \frac{\delta^{\prime}}{c}=\frac{\delta}{c^{2}} .
$$

Then there exist functions $\Phi=\Phi(\mathbf{v}) \in \mathcal{X}_{c \delta^{\prime}}, a=a(\mathbf{v}) \in X_{c \delta^{\prime}}$ and parameters $\alpha=\alpha(\mathbf{v}) \in$ $\left[-\delta^{\prime}, \delta^{\prime}\right], T=T(\mathbf{v}) \in\left[1-\delta^{\prime}, 1+\delta^{\prime}\right], x_{0}=x_{0}(\mathbf{v}) \in \mathbb{B}_{\delta^{\prime}}^{7}$ such that

$$
\Phi(\tau)=\mathbf{S}_{a_{\infty}}(\tau) \mathbf{U}\left(\mathbf{v}+\alpha \mathbf{h}, T, x_{0}\right)+\int_{0}^{\tau} \mathbf{S}_{a_{\infty}}(\tau-\sigma)\left[\mathbf{G}_{a(\sigma)}(\Phi(\sigma))-\partial_{\sigma} \Psi_{a(\sigma)}^{*}\right] d \sigma
$$

is satisfied for all $\tau \geq 0$. Moreover, the parameters depend Lipschitz continuously on $\mathbf{v}$ and

$$
\|\Phi(\tau)\| \lesssim e^{-\omega \tau}, \quad \forall \tau \geq 0 .
$$

It is easy to check that the function $\tilde{\mathbf{G}}(\sigma, \Phi)=\mathbf{G}_{a(\sigma)}(\Phi)-\partial_{\sigma} \Psi_{a(\sigma)}^{*}$ is continuously differentiable with respect to both variables ( $\Psi_{a}^{*}$ depends smoothly on $a \in X, \mathbf{F}_{a}(\mathbf{u})$ is algebraic in $\mathbf{u}$ and the remaining parts are bounded linear operators). Furthermore, $\mathbf{U}\left(\mathbf{v}+\alpha \mathbf{h}, T, x_{0}\right) \in$ $\mathcal{D}(\tilde{\mathbf{L}}) \subset \mathcal{D}(\mathbf{L})=\mathcal{D}\left(\mathbf{L}_{a_{\infty}}\right)$ for $\mathbf{v}$ smooth and standard semigroup theory, cf. for example Theorem 6.1.5 in [36], implies that $\Phi$ solves Eq. (2.9) and thus Eq. (2.6). By defining $\Psi(\tau):=\Psi_{a(\tau)}^{*}+\Phi(\tau)$ we get a solution $\Psi \in C^{1}([0, \infty), \mathcal{H})$ to

$$
\begin{aligned}
\partial_{\tau} \Psi(\tau) & =\mathbf{L} \Psi(\tau)+\mathbf{N}(\Psi(\tau)), \quad \tau>0 \\
\Psi(0) & =\mathcal{R}\left(\Psi_{0}^{*}, T, x_{0}\right)+\mathcal{R}\left(\mathbf{v}+\alpha \mathbf{h}, T, x_{0}\right) .
\end{aligned}
$$

Finally, by setting

$$
u(t, x):=\frac{1}{T-t} \psi_{1}(-\log (T-t)+\log T)\left(\frac{x-x_{0}}{T-t}\right),
$$

we obtain a solution to Eq. (1.9) in the backward lightcone $\bigcup_{t \in[0, T)}\{t\} \times \overline{\mathbb{B}_{T-t}^{7}\left(x_{0}\right)}$. It is left to show the claimed bounds. By definition, we have

$$
u_{T, x_{0}, a}^{*}(t, x)=\frac{1}{T-t} \psi_{a}^{*}\left(\frac{x-x_{0}}{T-t}\right) .
$$

Eq. (1.7) implies that $u_{T, x_{0}, a}^{*}$ blows up in $\dot{H}^{k}\left(\mathbb{B}_{T-t}^{7}\left(x_{0}\right)\right)$ for $k \geq 3$ with a rate given by $(T-$ $t)^{\frac{5}{2}-k}$, which yields the suitable normalization factor in the respective norms. In particular, we obtain

$$
\begin{aligned}
(T-t)^{\frac{1}{2}} \| & u(t, \cdot)-u_{T, x_{0}, a_{\infty}}^{*}(t, \cdot)\left\|_{\dot{H}^{3}\left(\mathbb{B}_{T-t}^{7}\left(x_{0}\right)\right)}=\right\| \psi_{1}(-\log (T-t)+\log T)-\psi_{a_{\infty}}^{*} \|_{\dot{H}^{3}\left(\mathbb{B}^{7}\right)} \\
& \leq\left\|\Psi(-\log (T-t)+\log T)-\Psi_{a_{\infty}}^{*}\right\| \\
& \lesssim\|\Phi(-\log (T-t)+\log T)\|+\left\|\Psi_{a(-\log (T-t)+\log T)}^{*}-\Psi_{a_{\infty}}^{*}\right\| \lesssim(T-t)^{\omega},
\end{aligned}
$$

where we have used Eqns. (2.3) and (6.1) in the last step. The other bounds follow analogously. 


\section{Appendix A. Proof of Lemma 3.1 And Lemma 3.2}

A.1. Proof of Lemma 3.1. By the definition

$$
\|u\|_{H^{2}\left(\mathbb{B}^{d}\right)}^{2}=\|u\|_{L^{2}\left(\mathbb{B}^{7}\right)}^{2}+\|\nabla u\|_{L^{2}\left(\mathbb{B}^{7}\right)}^{2}+\int_{\mathbb{B}^{7}} \partial_{i} \partial_{j} u(\xi) \overline{\partial^{i} \partial^{j} u(\xi)} d \xi
$$

and

$$
\|u\|_{H^{3}\left(\mathbb{B}^{d}\right)}^{2}=\|u\|_{L^{2}\left(\mathbb{B}^{7}\right)}^{2}+\|\nabla u\|_{L^{2}\left(\mathbb{B}^{7}\right)}^{2}+\int_{\mathbb{B}^{7}} \partial_{i} \partial_{j} u(\xi) \overline{\partial^{i} \partial^{j} u(\xi)} d \xi+\int_{\mathbb{B}^{7}} \partial_{i} \partial_{j} \partial_{k} u(\xi) \overline{\partial^{i} \partial^{j} \partial^{k} u(\xi)} d \xi .
$$

To proof the statement we use that for $u \in C^{1}\left(\overline{\mathbb{B}^{d}}\right)$

$$
\|u\|_{L^{2}\left(\mathbb{B}^{7}\right)}^{2} \lesssim\|\nabla u\|_{L^{2}\left(\mathbb{B}^{7}\right)}^{2}+\|u\|_{L^{2}\left(\mathbb{S}^{6}\right)}^{2},
$$

see for example Lemma 2.2, [2]. For $u \in C^{2}\left(\overline{\mathbb{B}^{7}}\right)$ this implies that

$$
\left\|\partial_{j} u\right\|_{L^{2}\left(\mathbb{B}^{7}\right)}^{2} \lesssim \int_{\mathbb{B}^{7}} \partial_{i} \partial_{j} u(\xi) \overline{\partial^{i} \partial_{j} u(\xi)} d \xi+\int_{\mathbb{S}^{6}} \partial_{j} u(\omega) \overline{\partial_{j} u(\omega)} d \sigma(\omega)
$$

for every $j=1, \ldots, 7$, such that

$$
\|\nabla u\|_{L^{2}\left(\mathbb{B}^{7}\right)}^{2} \lesssim \int_{\mathbb{B}^{7}} \partial_{i} \partial_{j} u(\xi) \overline{\partial^{i} \partial^{j} u(\xi)} d \xi+\int_{\mathbb{S}^{6}} \partial_{j} u(\omega) \overline{\partial^{j} u(\omega)} d \sigma(\omega) .
$$

Thus,

$$
\|u\|_{H^{2}\left(\mathbb{B}^{7}\right)}^{2} \lesssim \int_{\mathbb{B}^{7}} \partial_{i} \partial_{j} u(\xi) \overline{\partial^{i} \partial^{j} u(\xi)} d \xi+\int_{\mathbb{S}^{6}} \partial_{j} u(\omega) \overline{\partial^{j} u(\omega)} d \sigma(\omega)+\int_{\mathbb{S}^{6}}|u(\omega)|^{2} d \sigma(\omega) .
$$

Similarly, for $u \in C^{3}\left(\overline{\mathbb{B}^{7}}\right)$

$$
\left\|\partial_{j} \partial_{k} u\right\|_{L^{2}\left(\mathbb{B}^{7}\right)}^{2} \lesssim \int_{\mathbb{B}^{7}} \partial_{i} \partial_{j} \partial_{k} u(\xi) \overline{\partial^{i} \partial_{j} \partial_{k} u(\xi)} d \xi+\int_{\mathbb{S}^{6}} \partial_{j} \partial_{k} u(\omega) \overline{\partial_{j} \partial_{k} u(\omega)} d \sigma(\omega),
$$

which implies that

$$
\int_{\mathbb{B}^{7}} \partial_{i} \partial_{j} u(\xi) \overline{\partial^{i} \partial^{j} u(\xi)} d \xi \lesssim \int_{\mathbb{B}^{7}} \partial_{i} \partial_{j} \partial_{k} u(\xi) \overline{\partial^{i} \partial^{j} \partial^{k} u(\xi)} d \xi+\int_{\mathbb{S}^{6}} \partial_{i} \partial_{j} u(\omega) \overline{\partial^{i} \partial^{i} u(\omega)} d \sigma(\omega) .
$$

Hence,

$$
\begin{aligned}
\|u\|_{H^{3}\left(\mathbb{B}^{7}\right)}^{2} & \lesssim \int_{\mathbb{B}^{7}} \partial_{i} \partial_{j} \partial_{k} u(\xi) \overline{\partial^{i} \partial_{j} \partial_{k} u(\xi)} d \xi+\int_{\mathbb{S}^{6}} \partial_{i} \partial_{j} u(\omega) \overline{\partial^{i} \partial^{i} u(\omega)} d \sigma(\omega) \\
& +\int_{\mathbb{S}^{6}} \partial_{j} u(\omega) \overline{\partial^{j} u(\omega)} d \sigma(\omega)+\int_{\mathbb{S}^{6}}|u(\omega)|^{2} d \sigma(\omega),
\end{aligned}
$$

which proves that

$$
\|\mathbf{u}\|_{H^{3} \times H^{2}\left(\mathbb{B}^{7}\right)}^{2}=\left\|u_{1}\right\|_{H^{3}\left(\mathbb{B}^{7}\right)}^{2}+\left\|u_{2}\right\|_{H^{2}\left(\mathbb{B}^{7}\right)}^{2} \lesssim\|\mathbf{u}\|_{\mathcal{H}}
$$

for all $\mathbf{u}=\left(u_{1}, u_{2}\right) \in C^{3}\left(\overline{\mathbb{B}^{7}}\right) \times C^{2}\left(\overline{\mathbb{B}^{7}}\right)$. The reverse inequality follows from the trace theorem, see e.g. [23], p. 258, which asserts that

$$
\int_{\mathbb{S}^{7}}|u(\omega)|^{2} d \sigma(\omega) \lesssim\|u\|_{H^{1}\left(\mathbb{B}^{7}\right)}^{2},
$$

for all $u \in C^{1}\left(\overline{\mathbb{B}^{7}}\right)$. 
A.2. Proof of Lemma 3.2. In the following, we frequently use the identities

$$
2 \operatorname{Re}\left[\xi^{j} \partial_{j} f(\xi) \bar{f}(\xi)\right]=\partial_{\xi^{j}}\left[\xi^{j}|f(\xi)|^{2}\right]-d|f(\xi)|^{2}
$$

and

$$
\partial_{i_{1}} \partial_{i_{2}} \ldots \partial_{i_{k}}\left[\xi^{i} \partial_{i} f(\xi)\right]=k \partial_{i_{1}} \partial_{i_{2}} \ldots \partial_{i_{k}} f(\xi)+\xi^{i} \partial_{i} \partial_{i_{1}} \partial_{i_{2}} \ldots \partial_{i_{k}} f(\xi)
$$

for all $k \in \mathbb{N}$. With this the divergence theorem implies that

$$
\begin{aligned}
& \operatorname{Re} \int_{\mathbb{B}^{7}} \partial_{i} \partial_{j} \partial_{k}[\tilde{\mathbf{L}} \mathbf{u}]_{1}(\xi) \overline{\partial^{i} \partial^{j} \partial^{k} u_{1}(\xi)} d \xi=-\frac{1}{2} \int_{\mathbb{B}^{7}} \partial_{i} \partial_{j} \partial_{k} u_{1}(\xi) \overline{\partial^{i} \partial^{j} \partial^{k} u_{1}(\xi)} d \xi \\
& \quad+\operatorname{Re} \int_{\mathbb{B}^{7}} \partial_{i} \partial_{j} \partial_{k} u_{2}(\xi) \overline{\partial^{i} \partial^{j} \partial^{k} u_{1}(\xi)} d \xi-\frac{1}{2} \int_{\mathbb{S}^{6}} \partial_{i} \partial_{j} \partial_{k} u_{1}(\omega) \overline{\partial^{i} \partial^{j} \partial^{k} u_{1}(\omega)} d \sigma(\omega)
\end{aligned}
$$

Similarly,

$$
\begin{aligned}
& \operatorname{Re} \int_{\mathbb{B}^{7}} \partial_{i} \partial_{j}[\tilde{\mathbf{L} u}]_{2}(\xi) \overline{\partial^{i} \partial^{j} u_{2}(\xi)} d \xi=-\frac{1}{2} \int_{\mathbb{B}^{7}} \partial_{i} \partial_{j} u_{2}(\xi) \overline{\partial^{i} \partial^{j} u_{2}(\xi)} d \xi \\
& \quad-\operatorname{Re} \int_{\mathbb{B}^{7}} \partial_{i} \partial_{j} \partial_{k} u_{2}(\xi) \overline{\partial^{i} \partial^{j} \partial^{k} u_{1}(\xi)} d \xi-\frac{1}{2} \int_{\mathbb{S}^{6}} \partial_{i} \partial_{j} u_{2}(\omega) \overline{\partial^{i} \partial^{j} u_{2}(\omega)} d \sigma(\omega) \\
& \quad+\operatorname{Re} \int_{\mathbb{S}^{6}} \omega^{k} \partial_{k} \partial_{i} \partial_{j} u_{1}(\omega) \overline{\partial^{i} \partial^{j} u_{2}(\omega)} d \sigma(\omega)
\end{aligned}
$$

and

$$
\begin{aligned}
& \operatorname{Re} \int_{\mathbb{S}^{6}} \partial_{i} \partial_{j}[\tilde{\mathbf{L}} \mathbf{u}]_{1}(\omega) \overline{\partial^{i} \partial^{j} u_{1}(\omega)} d \sigma(\omega)=-3 \int_{\mathbb{S}^{6}} \partial_{i} \partial_{j} u_{1}(\omega) \overline{\partial^{i} \partial^{j} u_{1}(\omega)} d \sigma(\omega) \\
& \quad-\operatorname{Re} \int_{\mathbb{S}^{6}} \omega^{k} \partial_{k} \partial_{i} \partial_{j} u_{1}(\omega) \overline{\partial^{i} \partial^{j} u_{1}(\omega)} d \sigma(\omega)+\operatorname{Re} \int_{\mathbb{S}^{6}} \partial_{i} \partial_{j} u_{2}(\omega) \overline{\partial^{i} \partial^{j} u_{1}(\omega)} d \sigma(\omega) .
\end{aligned}
$$

Hence,

$$
\operatorname{Re}(\tilde{\mathbf{L}} \mathbf{u} \mid \mathbf{u})_{1}=-\frac{1}{2}(\mathbf{u} \mid \mathbf{u})_{1}-2 \int_{\mathbb{S}^{6}} \partial_{i} \partial_{j} u_{1}(\omega) \overline{\partial^{i} \partial^{j} u_{1}(\omega)} d \sigma(\omega)+\int_{\mathbb{S}^{6}} A(\omega) d \sigma(\omega)
$$

for

$$
\begin{aligned}
A(\omega) & =-\frac{1}{2} \partial_{i} \partial_{j} \partial_{k} u_{1}(\omega) \overline{\partial^{i} \partial^{j} \partial^{k} u_{1}(\omega)}-\frac{1}{2} \partial_{i} \partial_{j} u_{2}(\omega) \overline{\partial^{i} \partial^{j} u_{2}(\omega)}-\frac{1}{2} \partial_{i} \partial_{j} u_{1}(\omega) \overline{\partial^{i} \partial^{j} u_{1}(\omega)} \\
& +\operatorname{Re} \omega^{k} \partial_{k} \partial_{i} \partial_{j} u_{1}(\omega) \overline{\partial^{i} \partial^{j} u_{2}(\omega)}-\operatorname{Re} \omega^{k} \partial_{k} \partial_{i} \partial_{j} u_{1}(\omega) \overline{\partial^{i} \partial^{j} u_{1}(\omega)}+\operatorname{Re} \partial_{i} \partial_{j} u_{2}(\omega) \overline{\partial^{i} \partial^{j} u_{1}(\omega)} .
\end{aligned}
$$

An application of the inequality

$$
\operatorname{Re}(a \bar{b})+\operatorname{Re}(a \bar{c})-\operatorname{Re}(b \bar{c}) \leq \frac{1}{2}\left(|a|^{2}+|b|^{2}+|c|^{2}\right), \quad a, b, c \in \mathbb{C},
$$

shows that $A(\omega) \leq 0$. Analogously, one can show that

$$
\operatorname{Re}(\tilde{\mathbf{L}} \mathbf{u} \mid \mathbf{u})_{2}=-\frac{1}{2}(\mathbf{u} \mid \mathbf{u})_{2}-2 \int_{\mathbb{S}^{6}} \partial_{i} u_{2}(\omega) \overline{\partial^{i} u_{2}(\omega)} d \sigma(\omega)+\int_{\mathbb{S}^{6}} B(\omega) d \sigma(\omega)
$$

with $B(\omega) \leq 0$. Next, we consider

$$
\begin{aligned}
\operatorname{Re} \int_{\mathbb{S}^{6}} \partial_{i}[\tilde{\mathbf{L}} \mathbf{u}]_{1}(\omega) \overline{\partial^{i} u_{1}(\omega)} d \sigma(\omega) & =-2 \int_{\mathbb{S}^{6}} \partial_{i} u_{1}(\omega) \overline{\partial^{i} u_{1}(\omega)} d \sigma(\omega) \\
& -\operatorname{Re} \int_{\mathbb{S}^{6}} \omega^{k} \partial_{k} \partial_{i} u_{1}(\omega) \overline{\partial^{i} u_{1}(\omega)} d \sigma(\omega)+\operatorname{Re} \int_{\mathbb{S}^{6}} \partial_{i} u_{2}(\omega) \overline{\partial^{i} u_{1}(\omega)} d \sigma(\omega) .
\end{aligned}
$$


The Cauchy-Schwarz inequality implies that

$$
\begin{aligned}
& \operatorname{Re} \int_{\mathbb{S}^{6}}\left[\partial_{i} u_{2}(\omega)-\omega^{k} \partial_{k} \partial_{i} u_{1}(\omega)\right] \overline{\partial^{i} u_{1}(\omega)} d \sigma(\omega) \leq \frac{1}{2} \int_{\mathbb{S}^{6}} \partial_{i} u_{1}(\omega) \overline{\partial^{i} u_{1}(\omega)} d \sigma(\omega) \\
& \quad+\int_{\mathbb{S}^{6}} \partial_{i} u_{2}(\omega) \overline{\partial^{i} u_{2}(\omega)} d \sigma(\omega)+\int_{\mathbb{S}^{6}} \partial_{i} \partial_{j} u_{1}(\omega) \overline{\partial^{i} \partial^{j} u_{1}(\omega)} d \sigma(\omega)
\end{aligned}
$$

such that

$$
\begin{aligned}
\operatorname{Re} \int_{\mathbb{S}^{6}} \partial_{i}[\tilde{\mathbf{L} u}]_{1}(\omega) \overline{\partial^{i} u_{1}(\omega)} d \sigma(\omega) & \leq-\frac{3}{2} \int_{\mathbb{S}^{6}} \partial_{i} u_{1}(\omega) \overline{\partial^{i} u_{1}(\omega)} d \sigma(\omega)+\int_{\mathbb{S}^{6}} \partial_{i} u_{2}(\omega) \overline{\partial^{i} u_{2}(\omega)} d \sigma(\omega) \\
& +\int_{\mathbb{S}^{6}} \partial_{i} \partial_{j} u_{1}(\omega) \overline{\partial^{i} \partial^{j} u_{1}(\omega)} d \sigma(\omega) .
\end{aligned}
$$

Similarly,

$$
\begin{aligned}
\operatorname{Re} \int_{\mathbb{S}^{6}}[\tilde{\mathbf{L}} \mathbf{u}]_{2}(\omega) \overline{u_{2}(\omega)} d \sigma(\omega) & =-2 \int_{\mathbb{S}^{6}}\left|u_{2}(\omega)\right|^{2} d \sigma(\omega)+\operatorname{Re} \int_{\mathbb{S}^{6}} \Delta u_{1}(\omega) \overline{u_{2}(\omega)} d \sigma(\omega) \\
& -\operatorname{Re} \int_{\mathbb{S}^{6}} \omega^{k} \partial_{k} u_{2}(\omega) \overline{u_{2}(\omega)} d \sigma(\omega) \\
& \leq-\frac{3}{2} \int_{\mathbb{S}^{6}}\left|u_{2}(\omega)\right|^{2} d \sigma(\omega)+\int_{\mathbb{S}^{6}}\left|\Delta u_{1}(\omega)\right|^{2} d \sigma(\omega)+\int_{\mathbb{S}^{6}} \partial_{i} u_{2}(\omega) \overline{\partial^{i} u_{2}(\omega)} d \sigma(\omega),
\end{aligned}
$$

and

$$
\begin{aligned}
& \operatorname{Re} \int_{\mathbb{S}^{6}}[\tilde{\mathbf{L}} \mathbf{u}]_{1}(\omega) \overline{u_{1}(\omega)} d \sigma(\omega)=-\int_{\mathbb{S}^{6}}\left|u_{1}(\omega)\right|^{2} d \sigma(\omega)-\operatorname{Re} \int_{\mathbb{S}^{6}} \omega^{k} \partial_{k} u_{1}(\omega) \overline{u_{1}(\omega)} d \sigma(\omega) \\
& \quad+\operatorname{Re} \int_{\mathbb{S}^{6}} u_{2}(\omega) \overline{u_{1}(\omega)} d \sigma(\omega) \leq-\frac{1}{2} \int_{\mathbb{S}^{6}}\left|u_{1}(\omega)\right|^{2} d \sigma(\omega)+\int_{\mathbb{S}^{6}} \partial_{i} u_{1}(\omega) \overline{\partial^{i} u_{1}(\omega)} d \sigma(\omega)+\int_{\mathbb{S}^{6}}\left|u_{2}(\omega)\right|^{2} d \sigma(\omega) .
\end{aligned}
$$

In view of Eqn. (A.2) and (A.3) we obtain

$$
\begin{aligned}
\operatorname{Re}(\tilde{\mathbf{L}} \mathbf{u} \mid \mathbf{u}) & =4 \operatorname{Re}(\tilde{\mathbf{L}} \mathbf{u} \mid \mathbf{u})_{1}+\operatorname{Re}(\tilde{\mathbf{L}} \mathbf{u} \mid \mathbf{u})_{2}+\operatorname{Re} \int_{\mathbb{S}^{6}} \partial_{i}[\tilde{\mathbf{L}} \mathbf{u}]_{1}(\omega) \overline{\partial^{i} u_{1}(\omega)} d \sigma(\omega) v \\
& +\operatorname{Re} \int_{\mathbb{S}^{6}}[\tilde{\mathbf{L}} \mathbf{u}]_{2}(\omega) \overline{u_{2}(\omega)} d \sigma(\omega)+\operatorname{Re} \int_{\mathbb{S}^{6}}[\tilde{\mathbf{L} u}]_{1}(\omega) \overline{u_{1}(\omega)} d \sigma(\omega) \\
& \leq-2(\mathbf{u} \mid \mathbf{u})_{1}-\frac{1}{2}(\mathbf{u} \mid \mathbf{u})_{2} \\
& -\frac{1}{2} \int_{\mathbb{S}^{6}} \partial_{i} u_{1}(\omega) \overline{\partial^{i} u_{1}(\omega)} d \sigma(\omega)-\frac{1}{2} \int_{\mathbb{S}^{6}}\left|u_{2}(\omega)\right|^{2} d \sigma(\omega)-\frac{1}{2} \int_{\mathbb{S}^{6}}\left|u_{1}(\omega)\right|^{2} d \sigma(\omega) \\
& -7 \int_{\mathbb{S}^{6}} \partial_{i} \partial_{j} u_{1}(\omega) \overline{\partial^{i} \partial^{j} u_{1}(\omega)} d \sigma(\omega)+\int_{\mathbb{S}^{6}}\left|\Delta u_{1}(\omega)\right|^{2} d \sigma(\omega)
\end{aligned}
$$

An application of the Cauchy-Schwarz inequality shows that

$$
|\Delta u(\xi)|^{2}=\left|\sum_{i=1}^{7} \partial_{i}^{2} u(\xi)\right|^{2} \leq 7 \sum_{i=1}^{7}\left|\partial_{i}^{2} u(\xi)\right|^{2} \leq 7 \sum_{i, j=1}^{7}\left|\partial_{i} \partial_{j} u(\xi)\right|^{2}=7 \partial_{i} \partial_{j} u(\xi) \overline{\partial^{i} \partial^{j} u(\xi)}
$$

This finishes the proof. 


\section{ApPENDIX B. ExPLICIT EXPRESSIONS}

B.1. Expressions for Proposition 4.1. $C_{n}(\ell, \lambda)=P_{1}(n, \ell, \lambda) / P_{2}(n, \ell, \lambda)$ and $\varepsilon_{n}(\ell, \lambda)=$ $P_{3}(n, \ell, \lambda) / P_{2}(n, \ell, \lambda)$ where

$$
\begin{gathered}
P_{1}(n, \ell, \lambda)=16(2 n+2 \ell+7)(n+1)(2 n+\lambda+\ell+7)(2 n+\lambda+\ell-3), \\
P_{2}(n, \ell, \lambda)=\left[2 \lambda^{2}+4(4 n+2 \ell+9) \lambda+(2 n+2 \ell+9)(8 n+3 \ell)\right] . \\
\cdot\left[2 \lambda^{2}+4(4 n+2 \ell+5) \lambda+(2 n+2 \ell+7)(8 n+3 \ell-8)\right] . \\
P_{3}(n, \ell, \lambda)=\left(-16 n^{2}-28 n \ell-96 n+10 \ell-124\right) \lambda^{2} \\
+\left(-96 n^{2} \ell-48 n \ell^{2}-160 n^{2}+24 n \ell+104 \ell^{2}-640 n+20 \ell-568\right) \lambda \\
-(2 n+2 \ell+7)\left(24 n^{2} \ell+10 n \ell^{2}+64 n^{2}-96 n \ell-47 \ell^{2}+32 n+250 \ell-384\right) .
\end{gathered}
$$

$\delta_{3}(\ell, \lambda)=R_{1}(\ell, \lambda) / R_{2}(\ell, \lambda)$ where

$$
\begin{aligned}
R_{1}(\ell, \lambda)= & (23 \ell+190) \lambda^{6}-6\left(30 \ell^{2}+387 \ell+1094\right) \lambda^{5} \\
& +\left(-447 \ell^{3}-8670 \ell^{2}-46097 \ell-59746\right) \lambda^{4} \\
& -4\left(62 \ell^{4}+2935 \ell^{3}+26998 \ell^{2}+68573 \ell+8898\right) \lambda^{3} \\
& +\left(483 \ell^{5}-1770 \ell^{4}-97410 \ell^{3}-473652 \ell^{2}-209537 \ell+890030\right) \lambda^{2} \\
& +2\left(342 \ell^{6}+3735 \ell^{5}-8906 \ell^{4}-171178 \ell^{3}-181954 \ell^{2}+914227 \ell+77910\right) \lambda \\
& +3(\ell-1)\left(81 \ell^{6}+1427 \ell^{5}+5140 \ell^{4}-21610 \ell^{3}-81983 \ell^{2}+226647 \ell+314586\right), \\
R_{2}(\ell, \lambda)=2 \lambda^{8} & +16(2 \ell+11) \lambda^{7}+\left(216 \ell^{2}+2279 \ell+5526\right) \lambda^{6} \\
+ & 2\left(400 \ell^{3}+6066 \ell^{2}+27801 \ell+35914\right) \lambda^{5} \\
+ & \left(1772 \ell^{4}+34319 \ell^{3}+223270 \ell^{2}+532737 \ell+294126\right) \lambda^{4} \\
+ & 4\left(600 \ell^{5}+13922 \ell^{4}+114695 \ell^{3}+382702 \ell^{2}+370389 \ell-173626\right) \lambda^{3} \\
+ & \left(1944 \ell^{6}+51981 \ell^{5}+510802 \ell^{4}+2148322 \ell^{3}+2913884 \ell^{2}-2334671 \ell-3619670\right) \lambda^{2} \\
+ & 2\left(432 \ell^{7}+12978 \ell^{6}+146745 \ell^{5}+738530 \ell^{4}+1304890 \ell^{3}-1197446 \ell^{2}-3998275 \ell+420306\right) \lambda \\
+ & 3(\ell-1)(3 \ell+16)(13+2 \ell)\left(9 \ell^{5}+201 \ell^{4}+1410 \ell^{3}+2594 \ell^{2}-4235 \ell-4971\right) .
\end{aligned}
$$

$\delta_{5}(0, \lambda)=R_{3}(\lambda) / R_{4}(\lambda)$ where

$$
\begin{aligned}
R_{3}(\lambda)= & -191 \lambda^{8}-18994 \lambda^{7}-728158 \lambda^{6}-14060890 \lambda^{5}-149594764 \lambda^{4}-900471766 \lambda^{3} \\
& -3005668466 \lambda^{2}-4932933534 \lambda-2726072037 \\
R_{4}(\lambda)= & \lambda^{10}+184 \lambda^{9}+13910 \lambda^{8}+562738 \lambda^{7}+13346440 \lambda^{6}+191728906 \lambda^{5}+1667459514 \lambda^{4} \\
+ & 8524836246 \lambda^{3}+23936737079 \lambda^{2}+31789410678 \lambda+13392819504 .
\end{aligned}
$$

\section{B.2. Expressions for Proposition 4.2.}

$$
\begin{gathered}
\delta_{1}(\lambda)=\frac{2\left(\lambda^{3}+12 \lambda^{2}+140 \lambda+144\right)}{\lambda^{4}+50 \lambda^{3}+752 \lambda^{2}+3280 \lambda+4224}, \\
C_{n}(\lambda)=\frac{-4(n+1)(2 n+13)\left[\lambda^{2}+2(2 n+5) \lambda+4(n+3)(n+2)\right]}{\left[\lambda^{2}+4(2 n+7) \lambda+4(2 n+11)(n+2)\right]\left[\lambda^{2}+4(2 n+5) \lambda+4(2 n+9)(n+1)\right]},
\end{gathered}
$$




$$
\varepsilon_{n}(\lambda)=\frac{2\left[\lambda^{3}-2\left(n^{2}+5 n-5\right) \lambda^{2}-8\left(2 n^{2}-n-12\right) \lambda-8(2 n+3)(n+2)(n+1)\right]}{\left[\lambda^{2}+4(2 n+7) \lambda+4(2 n+11)(n+2)\right]\left[\lambda^{2}+4(2 n+5) \lambda+4(2 n+9)(n+1)\right]}
$$

\section{REFERENCES}

[1] Kendall Atkinson and Weimin Han. Spherical harmonics and approximations on the unit sphere: an introduction, volume 2044 of Lecture Notes in Mathematics. Springer, Heidelberg, 2012.

[2] P. Biernat, R. Donninger, and B. Schörkhuber. Hyperboloidal similarity coordinates and a globally stable blowup profile for supercritical wave maps. ArXiv e-prints, jul 2017.

[3] Paweł Biernat, Piotr Bizon, and Maciej Maliborski. Threshold for blowup for equivariant wave maps in higher dimensions. Nonlinearity, 30(4):1513-1522, 2017.

[4] P. Bizoń, P. Breitenlohner, D. Maison, and A. Wasserman. Self-similar solutions of the cubic wave equation. Nonlinearity, 23(2):225-236, 2010.

[5] Piotr Bizoń, Tadeusz Chmaj, and Zbisław Tabor. Dispersion and collapse of wave maps. Nonlinearity, 13(4):1411-1423, 2000.

[6] Piotr Bizoń, Tadeusz Chmaj, and Zbisław Tabor. On blowup for semilinear wave equations with a focusing nonlinearity. Nonlinearity, 17(6):2187-2201, 2004.

[7] Piotr Bizoń, Dieter Maison, and Arthur Wasserman. Self-similar solutions of semilinear wave equations with a focusing nonlinearity. Nonlinearity, 20(9):2061-2074, 2007.

[8] Annegret Y. Burtscher and Roland Donninger. Hyperboloidal evolution and global dynamics for the focusing cubic wave equation. Comm. Math. Phys., 353(2):549-596, 2017.

[9] S. Burzio and J. Krieger. Type II blow up solutions with optimal stability properties for the critical focussing nonlinear wave equation on $\mathrm{R}^{\wedge}\{3+1\}$. ArXiv e-prints, September 2017.

[10] A. Chatzikaleas and R. Donninger. Stable blowup for the cubic wave equation in higher dimensions. ArXiv e-prints, December 2017.

[11] Charles Collot. Type II blow up manifolds for the energy supercritical semilinear wave equation. Mem. Amer. Math. Soc., 252(1205):v+163, 2018.

[12] Ovidiu Costin, Roland Donninger, and Irfan Glogić. Mode stability of self-similar wave maps in higher dimensions. Comm. Math. Phys., 351(3):959-972, 2017.

[13] Ovidiu Costin, Roland Donninger, Irfan Glogić, and Min Huang. On the stability of self-similar solutions to nonlinear wave equations. Comm. Math. Phys., 343(1):299-310, 2016.

[14] Benjamin Dodson and Andrew Lawrie. Scattering for radial, semi-linear, super-critical wave equations with bounded critical norm. Arch. Ration. Mech. Anal., 218(3):1459-1529, 2015.

[15] Roland Donninger. Strichartz estimates in similarity coordinates and stable blowup for the critical wave equation. Duke Math. J., 166(9):1627-1683, 2017.

[16] Roland Donninger and Irfan Glogić. On the existence and stability of blowup for wave maps into a negatively curved target. Anal. PDE, 12(2):389-416, 2019.

[17] Roland Donninger and Birgit Schörkhuber. Stable self-similar blow up for energy subcritical wave equations. Dyn. Partial Differ. Equ., 9(1):63-87, 2012.

[18] Roland Donninger and Birgit Schörkhuber. On blowup in supercritical wave equations. Comm. Math. Phys., 346(3):907-943, 2016.

[19] Roland Donninger and Birgit Schörkhuber. Stable blowup for wave equations in odd space dimensions. Ann. Inst. H. Poincaré Anal. Non Linéaire, 34(5):1181-1213, 2017.

[20] Roland Donninger and Anıl Zenginoğlu. Nondispersive decay for the cubic wave equation. Anal. PDE, 7(2):461-495, 2014.

[21] Saber Elaydi. An introduction to difference equations. Undergraduate Texts in Mathematics. Springer, New York, third edition, 2005.

[22] Klaus-Jochen Engel and Rainer Nagel. One-parameter semigroups for linear evolution equations, volume 194 of Graduate Texts in Mathematics. Springer-Verlag, New York, 2000. With contributions by S. Brendle, M. Campiti, T. Hahn, G. Metafune, G. Nickel, D. Pallara, C. Perazzoli, A. Rhandi, S. Romanelli and R. Schnaubelt.

[23] Lawrence C. Evans. Partial Differential Equations. Graduate Studies in Mathematics. American Mathematical Society, second edition, 2010. 
[24] Carsten Gundlach and José M. Martín-García. Critical phenomena in gravitational collapse. Living Reviews in Relativity, 10(1):5, Dec 2007.

[25] Matthieu Hillairet and Pierre Raphaël. Smooth type II blow-up solutions to the four-dimensional energycritical wave equation. Anal. PDE, 5(4):777-829, 2012.

[26] Tosio Kato. Perturbation theory for linear operators. Classics in Mathematics. Springer-Verlag, Berlin, 1995. Reprint of the 1980 edition.

[27] Carlos E. Kenig and Frank Merle. Global well-posedness, scattering and blow-up for the energy-critical focusing non-linear wave equation. Acta Math., 201(2):147-212, 2008.

[28] J. Krieger. On stability of type II blow up for the critical NLW on $\backslash \mathrm{R}^{\wedge}\{3+1\}$. ArXiv e-prints, May 2017.

[29] J. Krieger and W. Schlag. On the focusing critical semi-linear wave equation. Amer. J. Math., 129(3):843-913, 2007.

[30] Joachim Krieger, Kenji Nakanishi, and Wilhelm Schlag. Global dynamics away from the ground state for the energy-critical nonlinear wave equation. Amer. J. Math., 135(4):935-965, 2013.

[31] Joachim Krieger, Kenji Nakanishi, and Wilhelm Schlag. Global dynamics of the nonradial energy-critical wave equation above the ground state energy. Discrete Contin. Dyn. Syst., 33(6):2423-2450, 2013.

[32] Joachim Krieger, Kenji Nakanishi, and Wilhelm Schlag. Threshold phenomenon for the quintic wave equation in three dimensions. Comm. Math. Phys., 327(1):309-332, 2014.

[33] Joachim Krieger, Kenji Nakanishi, and Wilhelm Schlag. Center-stable manifold of the ground state in the energy space for the critical wave equation. Math. Ann., 361(1-2):1-50, 2015.

[34] Howard A. Levine. Instability and nonexistence of global solutions to nonlinear wave equations of the form $P u_{t t}=-A u+\mathcal{F}(u)$. Trans. Amer. Math. Soc., 192:1-21, 1974.

[35] Frank Merle and Hatem Zaag. Determination of the blow-up rate for a critical semilinear wave equation. Math. Ann., 331(2):395-416, 2005.

[36] Amnon Pazy. Semigroups of linear operators and applications to partial differential equations. Applied Mathematical Sciences. Springer, New York, Berlin, 1983.

[37] Jan Prüss. On the spectrum of $C_{0}$-semigroups. Trans. Amer. Math. Soc., 284(2):847-857, 1984.

[38] Barry Simon. Operator theory. A Comprehensive Course in Analysis, Part 4. American Mathematical Society, Providence, RI, 2015.

[39] H. S. Wall. Polynomials whose zeros have negative real parts. Amer. Math. Monthly, 52:308-322, 1945.

Universität Wien, Fakultät für Mathematik, Oskar-Morgenstern-Platz 1, A-1090 Vienna, Austria

E-mail address: irfan.glogic@univie.ac.at

The Ohio State University, Department of Mathematics, 231 West 18th Ave, Columbus, USA

E-mail address: glogic.1@osu.edu

Karlsruhe Institute of Technology, Institute for Analysis, Englerstrasse 2, 76131 KarlSRUHe, GERMANY

E-mail address: birgit.schoerkhuber@kit.edu 